\title{
Estructura de grupos finitos y propiedades aritméticas de los tamaños de clase de conjugación
}

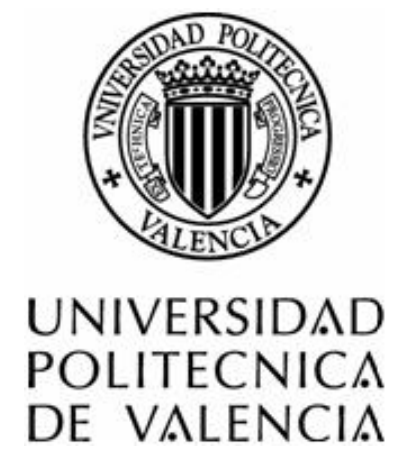

Elena Alemany Martínez ealemany@mat.upv.es

Departamento de Matemática Aplicada

Universidad Politécnica de Valencia

Camino de Vera s/n. 46022 Valencia

Memoria presentada por $D^{\text {ña }}$ Elena Alemany Martínez para optar al grado de Doctor Trabajo dirigido por el Dr. Antonio Beltrán Felip y la Dra. María José Felipe Román 

D. Antonio Beltrán Felip, profesor Titular de Universidad del Departamento de Matemáticas de la Universidad Jaume I de Castellón, y $\mathrm{D}^{\tilde{n} a}$ María José Felipe Román, profesora Titular de Universidad del Departamento de Matemática Aplicada de la Universidad Politécnica de Valencia.

\section{CERTIFICAN QUE:}

La presente Memoria, titulada "Estructura de grupos finitos y propiedades aritméticas de los tamaños de clase de conjugación", ha sido realizada bajo nuestra dirección por $\mathrm{D}^{\text {ña }}$ Elena Alemany Martínez para optar al grado de Doctor en Matemáticas.

Lo que hacemos constar en cumplimiento de la legislación vigente.

Valencia, 3 de mayo de 2011

Fdo. Antonio Beltrán Felip

Fdo. María José Felipe Román 



\section{Agradecimientos}

Mis agradecimientos son, sin duda alguna, para $\mathrm{M}^{\mathrm{a}}$ José Felipe Román y Antonio Beltrán Felip, que me dieron la oportunidad de trabajar con ellos, introduciéndome y formándome en un área de investigación básicamente nueva para mi.

A $\mathrm{M}^{\mathrm{a}}$ José, amiga y compañera de trabajo, quiero agradecerle especialmente la confianza que en su día depositó en mí, al ofrecerme la posibilidad de trabajar en este proyecto, y posteriormente, prepararme para llevarlo a cabo. Su fortaleza y motivación, incluso en los momentos más difíciles, han sido para mí un claro referente, tanto a nivel profesional como personal.

A Antonio, quiero agradecerle el haberme tratado siempre como un amigo y compañero con el que he podido contar en todo momento. Su asesoramiento y su refuerzo, siempre positivos, me han proporcionado la motivación y la confianza necesarias para concluir este proyecto, permitiéndome disfrutar y al mismo tiempo formarme como investigador.

A Ana Martínez Pastor, quiero agradecerle sus consejos, así como el cariño y apoyo que me ha mostrado durante los últimos meses. 

A Carolina y a Laura, y con especial cariño y gratitud a Quique 



\section{Índice general}

Resúmenes $\quad$ XI

Introducción $\quad$ XIX

1. Tamaños de clases de conjugación y estructura de grupos 1

2. Resultados previos $\quad 9$

3. Grupos con dos tamaños de clases de elementos p-regulares 17

3.1. Introducción . . . . . . . . . . . . . . . . . . . 17

3.2. Resultados preliminares . . . . . . . . . . . . . . . 19

3.3. Resultado Principal . . . . . . . . . . . . . . . . 23

4. Grupos con dos tamaños de clases de elementos de orden potencia $\begin{array}{ll}\text { de primo } & 39\end{array}$

4.1. Introducción . . . . . . . . . . . . . . . . . . . . . . 39

4.2. Resultados preliminares . . . . . . . . . . . . . . 40

4.3. Resultados Principales . . . . . . . . . . . . . . . 43

5. Subgrupos normales con dos tamaños de $G$-clases de conjugación 49

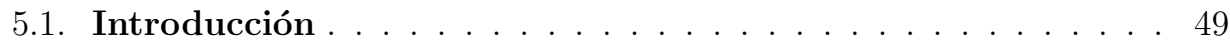

5.2 . Resultados preliminares $\ldots \ldots \ldots \ldots \ldots$

5.3. Resultados Principales . . . . . . . . . . . . . . 53

$\begin{array}{ll}\text { Bibliografía } & 63\end{array}$

$\begin{array}{ll}\text { Notación } & 69\end{array}$ 



\section{Resúmenes de tesis}

\section{Resumen}

La presente memoria se desarrolla dentro del marco de la Teoría de Grupos Finitos; concretamente se estudia la relación existente entre la estructura de un grupo y los tamaños de clases de conjugación de sus elementos. La obtención de información sobre las propiedades estructurales del grupo a partir de sus tamaños de clases de conjugación es un campo clásico en la Teoría de Grupos Finitos. En los últimos años se han abierto nuevas líneas de investigación que dan cabida al estudio estructural del grupo a partir de los tamaños de clases de ciertos subconjuntos de elementos del grupo. Es el caso de los elementos reales, los elementos racionales, los elementos de orden potencia de primo, los elementos $p$-regulares y los elementos de un subgrupo normal del grupo, entre otros. Se trata de un área poco investigada, cuyos resultados tienen aplicación en el propio campo, en la obtención de información sobre la estructura de los grupos y de sus subgrupos, al considerar los tamaños de sus clases ordinarias.

En este trabajo estudiamos los tamaños de clase de conjugación de los elementos $p$-regulares del grupo y de los elementos de orden potencia de primo. Asímismo, se investiga la influencia de los tamaños de $G$-clases de los elementos de un subgrupo normal en la estructura de dicho subgrupo. Partiendo de un teorema clásico para el caso ordinario, como es el Teorema de Itô ([43]), que establece la nilpotencia de un grupo con dos tamaños de clases de conjugación, se desarrollan nuevas demostraciones que permiten extender este resultado, cuando consideramos algunos subconjuntos de elementos del grupo. Al realizar dichas extensiones utilizamos, en la medida de lo posible, técnicas sencillas que no requieran resultados profundos.

El primer capítulo de la memoria es una recopilación de conceptos y resultados básicos sobre tamaños de clase de conjugación en un grupo finito. En él se realiza un "survey" que recoge algunos de los resultados más importantes conocidos hasta 
el momento, y que constituyen el punto de partida de nuestra investigación.

En el segundo capítulo se recogen los resultados preliminares que hemos necesitado para abordar los problemas planteados sobre los tamaños de clases de conjugación. Los resultados estándar de la teoría de grupos sólo se enuncian, mientras que aquellos resultados menos conocidos que hemos necesitado para desarrollar nuestro trabajo, se incluyen con demostración.

Nuestros resultados quedan recogidos en los tres últimos capítulos de la memoria. El tercer capítulo está dedicado al estudio de la $p$-estructura del grupo a partir de los tamaños de clase de conjugación de sus elementos p-regulares. En [7], A. Beltrán y M.J. Felipe obtienen una generalización del Teorema de Itô para tamaños de clases de conjugación de elementos $p$-regulares, para un cierto primo $p$, bajo la hipótesis de $p$-resolubilidad del grupo. En este capítulo se presenta una demostración conjunta de los resultados obtenidos en [7] y [2], que establece que si $G$ es un grupo finito con tamaños de clase de conjugación de elementos $p$-regulares 1 y $m$, para algún entero $m$, entonces $m=p^{a} q^{b}$, con $p$ un primo distinto de $q$, y $a, b \geq 0$. Se demuestra que si $b=0$ entonces el grupo $G$ tiene $p$-complemento abeliano, y si $b \neq 0$ entonces $G=P Q \times A$, con $P$ un $p$-subgrupo de Sylow de $G, Q$ un $q$-subgrupo de Sylow de $G$ y $A \leq \mathbf{Z}(G)$. Asímismo, se demuestra que si $a=0$ entonces $G=P \times Q \times A$, con $A \leq \mathbf{Z}(G)$. En particular, se obtiene que $G$ es resoluble.

En el cuarto capítulo se investiga la estructura de los grupos a partir de los tamaños de clases de conjugación de elementos de orden potencia de primo y se presentan los principales resultados recogidos en [4]. El resultado principal del capítulo establece que si $G$ es un grupo p-resoluble con tamaños de clase de conjugación de $p^{\prime}$-elementos de orden potencia de primo 1 y $m$, entonces $m=p^{a} q^{b}$ con $q$ un primo distinto de $p$, y $a, b \geq 0$. Se demuestra que si $b=0$ entonces $G$ tiene $p$-complementos abelianos, y si $b \neq 0$ entonces $G=P Q \times A$, con $P$ un $p$-subgrupo de Sylow de $G, Q$ un $q$-subgrupo de Sylow de $G$ y $A \subseteq \mathbf{Z}(G)$. Obtenemos por tanto, una generalización del teorema principal de [7] para elementos de orden potencia de primo. Concluimos el capítulo aplicando el anterior resultado al estudio de la estructura de un grupo $G$ con dos tamaños de clases de conjugación de elementos de orden potencia de primo. Partiendo de la resolubilidad del grupo, se demuestra que $G$ es de la forma $G=P \times A$, con $P$ un $p$-subgrupo de Sylow de $G$ y $A$ un subgrupo abeliano; por consiguiente, el grupo es nilpotente. Se obtiene así una generalización del Teorema de Itô para elementos de orden potencia de primo. Obsérvese que para obtener las citadas generalizaciones se presentan demostraciones alternativas del resultado de Itô y del teorema principal de [7] para grupos resolubles.

En el quinto y último capítulo se estudia la estructura de los subgrupos normales 
de un grupo, bajo ciertas condiciones aritméticas sobre los tamaños de las $G$-clases de conjugación contenidas en dichos subgrupos. En particular se demuestra que si $N$ es un subgrupo normal de $G$ tal que los tamaños de $G$-clases de $N$ son 1 y $m$, para algún entero $m$, entonces $N$ es abeliano ó es producto directo de un $p$-grupo no abeliano por un subgrupo central de $G$, y por tanto, es nilpotente. La conclusión final se obtiene demostrando primero la nilpotencia en el universo resoluble, y extendiendo el resultado al caso no resoluble. La demostración del caso no resoluble se apoya en el conocimiento de la estructura de los $C P$-grupos simples, determinada recientemente por Heineken. Concluimos el capítulo extendiendo una propiedad de los $p$-grupos con dos tamaños de clases, a los $p$-subgrupos normales no abelianos de un grupo $G$ con dos tamaños de $G$-clases. Concretamente demostramos que si $G$ es un grupo finito y $P$ es un $p$-subgrupo normal no abeliano de $G$, con dos tamaños de $G$-clases de conjugación, entonces $P /(\mathbf{Z}(G) \cap P)$, y en particular $P / \mathbf{Z}(P)$, tienen exponente $p$. Todos estos resultados aparecen publicados en [3]. 


\section{Summary}

This essay is developed within the context of the Finite Group Theory. It studies the relationship between the structure of a group and the conjugacy class sizes of its elements. The search of information about the structural properties of a group from its conjugacy class sizes is a traditional problem in Finite Group Theory. In the last few years, new lines of investigation have emerged to include the structural analysis of the groups from the conjugacy class sizes of given subsets of elements of the group. This is the case of real elements, rational elements, elements of prime power order, $p$-regular elements or elements of a normal subgroup of the group, among others. This is an under-researched field and the results can be applied again to get information on the structure of a group or a subgroup, from its ordinary conjugacy class sizes.

In this research we study the conjugacy class sizes of $p$-regular elements y elements of prime power order. We also investigate the influence of the conjugacy $G$-class sizes of the elements of a normal subgroup on its structure. From a classical theorem for the ordinary case, as it is Itô's Theorem, which establishes the nilpotency of a group with two conjugacy class sizes, we develop new proofs in order to extend this result, when the above mentioned subsets of elements of the group are considered. While making such extensions we use, when possible, straightforward methods which do not require deep or complex results.

In chapter one of the essay we make a short review of the concepts and basic results on conjugacy class sizes of a group. It includes a survey which collects some of the most important results known so far, as a starting point of our investigation.

The second chapter contains all the preliminary results which we have needed to deal with the different problems on conjugacy class sizes we have treated. Standard results are only stated, while those results, less common, that we have used to develop our work, are provided with demonstration.

Most of the proofs are provided, so as to obtain a self-contained document, but some well-known results results are only stated, since the demonstrations are out of our framework.

Our findings are collected in the last three chapters of the essay. The third chapter is dedicated to the study of the $p$-structure of the group from the conjugacy class sizes of its $p$-regular elements. In [7], A. Beltrán and M.J. Felipe prove a generalization of Itô's Theorem for class sizes of $p$-regular elements, for some prime $p$, 
given the hypothesis of $p$-solvability of the group. In this chapter we provide a joined demonstration of the results obtained in [7] and [2], which shows that if $G$ is a finite group with conjugacy class sizes of $p$-regular elements 1 and $m$, for some integer $m$, then $m=p^{a} q^{b}$, with $p$ and $q$ two different primes, and $a, b \geq 0$. It is also shown that if $b=0$ then the group $G$ has abelian $p$-complement, and if $b \neq 0$ then $G=P Q \times A$, with $P$ a Sylow $p$-subgroup of $G, Q$ a Sylow $q$-subgroup of $G$ and $A \leq \mathbf{Z}(G)$. It is proved as well that if $a=0$ then $G=P \times Q \times A$, with $A \leq \mathbf{Z}(G)$. In particular, it is deduced that $G$ is solvable.

In chapter four we investigate the structure of the groups from the conjugacy class sizes of elements of prime power order and expose the main results obtained in [4]. The main result of the chapter asserts that if $G$ is a $p$-solvable group with conjugacy class sizes of $p^{\prime}$-elements of prime power order 1 and $m$, then $m=p^{a} q^{b}$ with $q$ and $p$ two different primes, and $a, b \geq 0$. It is shown that if $b=0$ then $G$ has abelian $p$-complements, and if $b \neq 0$ then $G=P Q \times A$, with $P$ a Sylow $p$-subgroup of $G, Q$ a Sylow $q$-subgroup of $G$ and $A \subseteq \mathbf{Z}(G)$. Therefore we get a new generalization of the main theorem in [7] for elements of prime power order. We end the chapter by applying the latter result to the study of the structure of a group $G$ with two conjugacy class sizes of elements of prime power order. Given that such a group is solvable, we prove that it can be written as a product $G=P \times A$, with $P$ a Sylow $p$-subgroup of $G$ and $A$ an abelian subgroup; therefore, the group is nilpotent. This is a new generalization of Itô's Theorem for elements of prime power order. Notice that in order to obtain the mention generalizations, we provide alternative proofs of Itô's Theorem and of the main theorem of [7] for the class of solvable groups.

In chapter five we investigate the structure of the normal subgroups of a group, under determined arithmetical conditions on the conjugacy $G$-class sizes contained in such subgroups. In particular, it is shown that if $N$ is a normal subgroup of $G$ such that the conjugacy $G$-class sizes in $N$ are 1 and $m$, for some integer $m$, then $N$ is abelian or $N$ is a direct product of a non abelian $p$-group by a central subgroup of $G$, and therefore, is nilpotent. The final conclusion is obtained by proving first the nilpotency in the solvable universe, and extending the result to the non solvable case. The proof of the non solvable case relies on the knowledge of the structure of simple CP-groups, recently determined by Heineken. We conclude the chapter extending a property of $p$-groups with two conjugacy class sizes, to normal non abelian $p$-subgroups of a group $G$ with two conjugacy $G$-class sizes. Specifically we show that if $G$ is a finite group and $P$ is a normal non abelian $p$-subgroup of $G$, with two conjugacy $G$-class sizes, then $P /(\mathbf{Z}(G) \cap P)$, and in particular $P / \mathbf{Z}(P)$, have exponent $p$. All these results have been published in [3]. 


\section{Resum}

La present memòria es desenvolupa dins del marc de la Teoria de Grups Finits, concretament en l'estudi de la relació existent entre l'estructura d'un grup i les mides de classes de conjugació dels seus elements. L'obtenció d'informació sobre les propietats estructurals del grup a partir de les seues classes de conjugació és un camp clàssic en la Teoria de Grups Finits. En els últims anys s'han obert noves línies d'investigació que donen cabuda a l'estudi estructural del grup a partir de les mides de classes de certs subconjunts d'elements del grup. És el cas dels elements reals, els elements racionals, els elements d'ordre potència de primer, els elements $p$-regulars $\mathrm{i}$ els elements d'un subgrup normal, entre altres. Es tracta d'un àrea poc investigada, quins resultats tenen aplicació en el propi camp, en l'obtenció d'informació sobre l'estructura dels grups i dels seus propis subgrups, al considerar les mides de les seues classes ordinàries.

En aquest treball estudiem les mides de classe de conjugació dels elements $p$ regulars del grup i dels elements d'ordre potència de primer. Així mateix, s'investiga la influència de les mides de $G$-classes dels elements d'un subgrup normal en l'estructura d'aquest subgrup. Partint d'un teorema clàssic per al cas ordinari, com és el Teorema d'Ito, que estableix la nilpotència d'un grup amb dues mides de classes de conjugació, es desenvolupen noves demostracions que permeten estendre aquet resultat, quan considerem alguns subconjunts d'elements del grup. Al realitzar aquestes extensions utilitzem, en la mesura del possible, tècniques senzilles que no requereixen resultats profunds.

El primer capítol de la memòria és una recopilació de conceptes i resultats bàsics sobre mides de classe de conjugació en un grup finit. En ell es realitza un "survey"que recull alguns dels resultats més importants coneguts fins al moment, i que constitueixen el punt de partida de la nostra investigació.

En el segon capítol es recullen aquells resultats preliminars que hem necessitat per abordar els problemes plantejats sobre les mides de classes de conjugació. Els resultats estàndard de la teoria de grups només s'enuncien, mentres que aquells resultats meyns conneguts que hem necessitat per desenvolupar el nostre treball, s'inclouen com a demostració.

Els nostres resultats estan recollits en els tres últims capítols de la memòria. El tercer capítol està dedicat a l'estudi de la p-estructura del grup a partir de les mides de classe de conjugació dels seus elements p-regulars. En [7], A. Beltrán i M. J. Felipe obtenen una generalització del Teorema d'Ito per a mides de classe de conjugació d'elements $p$-regulars, per a un cert primer $p$, baix la hipòtesi de $p$ - 
resolubilitat del grup. En aquest capítol es presenta una demostració conjunta dels resultats obtinguts en [7] i en [2], que estableix que si $G$ és un grup finit amb mides de classe de conjugació d'elements $p$-regulars 1 i $m$, per a algun enter $m$, aleshores $m=p^{a} q^{b}$, amb $p$ un primer distint de $q$, i $a, b \geq 0$. Es demostra que si $b=0$ aleshores el grup $G$ té $p$-complement abelià i si $b \neq 0$ aleshores $G=P Q \times A$, amb $P$ un $p$ subgrup de Sylow de $G, Q$ un $q$-subgrup de Sylow de $G$ i $A \leq \mathbf{Z}(G)$. Així mateix es demostra que si $a=0$ aleshores $G=P \times Q \times A$, amb $A \leq \mathbf{Z}(G)$. En particular, s'obté que $G$ és resoluble.

En el quart capítol s'investiga l'estructura dels grups a partir de les mides de classes de conjugació d'elements d'ordre potència de primer i es presenten els principals resultats recollits en [4]. El resultat principal del capítol estableix que si $G$ és un grup $p$-resoluble amb mides de classe de conjugació de $p^{\prime}$-elements d'ordre potència de primer $1 \mathrm{i} m$, aleshores $m=p^{a} q^{b}$ amb $q$ un primer distint de $p$, i $a, b \geq 0$. Es demostra que si $b=0$ aleshores $G$ té $p$-complements abelians, i si $b \neq 0$ aleshores $G=P Q \times A$, amb $P$ un $p$-subgrup de Sylow de $G, Q$ un $q$-subgrup de Sylow de $G$ i $A<\mathbf{Z}(G)$. Obtenim per tant, una generalització del teorema principal de [7] per a elements d'ordre potència de primer. Concloem el capítol aplicant l'anterior resultat a l'estudi de l'estructura d'un grup $G$ amb dues mides de classes de conjugació d'elements d'ordre potència de primer. Partint de la resolubilitat del grup, es demostra que $G$ és de la forma $G=P \times A$, amb $P$ un $p$-subgrup de Sylow de $G$ i $A$ un subgrup abelià; llavors, el grup és nilpotent. S'obté així una generalització del Teorema d'Ito per a elements d'ordre potència de primer. Observeu que per a obtenir les citades generalitzacions es presenten demostracions alternatives del resultat d'Ito i del teorema principal de [7] per a grups resolubles.

En el quint i últim capítol s'estudia l'estructura dels subgrups normals d'un grup, baix certes condicions aritmètiques sobre les mides de les $G$-classes de conjugació contingudes en aquests subgrups. En particular es demostra que si $N$ és un subgrup normal de $G$ tal que les mides de $G$-classes de $N$ són 1 i $m$, per a algun enter $m$, aleshores $N$ és abelià o és producte directe d'un $p$-grup no abelià per un subgrup central de $G$, i per tant, és nilpotent. La conclusió final s'obté demostrant primer la nilpotència en l'univers resoluble, i estenent el resultat al cas no resoluble. La demostració del cas no resoluble es recolza en el coneixement de l'estructura dels $C P$-grups simples, determinada recentment per Heineken. Concloem el capítol estenent una propietat dels $p$-grups amb dues mides de classes, als $p$-subgrups normals no abelians d'un grup $G$ amb dues mides de $G$-classes. Concretament demostrem que si $G$ és un grup finit i $P$ és un $p$-subgrup normal no abelià de $G$, amb dues mides de $G$-classes de conjugació, aleshores $P /(\mathbf{Z}(G) \cap P)$, i en particular $P / \mathbf{Z}(P)$, tenen exponent $p$. Tots aquestos resultats apareixen publicats en [3]. 



\section{Introducción}

La presente memoria se desarrolla dentro del marco de la Teoría de Grupos Finitos, concretamente en el estudio de la relación existente entre la estructura de un grupo y los tamaños de clases de conjugación de sus elementos.

La obtención de información sobre propiedades estructurales de un grupo a partir de sus clases de conjugación es un tema clásico. Los primeros resultados se deben a M.L. Sylow y W. Burnside. Sylow demuestra que un grupo cuyos tamaños de clase son todos potencia de un cierto primo $p$ tiene centro no trivial, mientras que Burnside prueba que si el grupo $G$ tiene un tamaño de clase de conjugación potencia de un primo, entonces es no simple. Posteriormente, en 1990, L. S. Kazarin obtiene una extensión de este resultado demostrando que en tales circunstancias el grupo generado por la clase de conjugación de dicho elemento es un subgrupo normal resoluble de $G$.

En la década de los 90, tras una etapa de evolución y desarrollo de la Teoría General de Grupos, renace el interés por el estudio de las propiedades aritméticas de las clases de conjugación y su influencia en la estructura del grupo. Lejos de ser un tema cerrado, en los últimos años se han obtenido nuevos e interesantes resultados, abriéndose líneas de investigación alternativas. Se consideran los tamaños de clases de los elementos reales, los elementos racionales, los elementos de orden potencia de primo, los elementos p-regulares y los elementos de un subgrupo normal del grupo, por citar algunos. Se trata de un área de investigación con numerosos problemas abiertos y muchos de los resultados conocidos tienen aplicación en el propio campo, en la obtención de información sobre la estructura de los grupos y de sus propios subgrupos, al considerar los tamaños de sus clases ordinarias.

En este trabajo se estudian los tamaños de clase de conjugación de los elementos $p$-regulares y los elementos de orden potencia de primo (también llamados elementos primarios). Asimismo, se investiga la influencia de los tamaños de $G$-clases de los elementos de un subgrupo normal en la estructura de dicho subgrupo. Partiendo de un teorema clásico para el caso ordinario, como es el Teorema de Itô ([43]), que afirma que un grupo con dos tamaños de clases de conjugación es, salvo factores centrales, un $p$-grupo, para un cierto primo $p$, se desarrollan nuevas demostraciones 
que permiten extender este resultado cuando se consideran los citados subconjuntos de elementos del grupo. Al realizar dichas extensiones utilizamos, en la medida de lo posible, técnicas sencillas que no requieran resultados profundos. Hemos podido comprobar que las generalizaciones que se obtienen aportan información relevante sobre la estructura del grupo. También hemos manejado situaciones nuevas que han requerido ser tratadas con nuevas técnicas, todo lo cual aportamos como parte de nuestra investigación.

La memoria consta de cinco capítulos. Todos ellos estudian diferentes extensiones del citado teorema de Itô en diversos contextos. Comenzaremos haciendo un resumen en el primer capítulo de algunos de los resultados más importantes que se conocen hasta el momento, y que determinan el estado actual de las investigaciones sobre tamaños de clase de conjugación y su influencia en el estudio de la estructura del grupo.

El segundo capítulo recopila aquellos resultados preliminares que hemos necesitado para abordar los problemas planteados en los siguientes capítulos de la memoria. Omitimos la demostración de aquellos resultados que son estándar en la Teoría General de Grupos.

El tercer capítulo de la memoria está dedicado al estudio de la $p$-estructura del grupo a partir de los tamaños de clases de conjugación de sus elementos p-regulares. Obsérvese que este estudio no es sencillo, incluso bajo la hipótesis de $p$-resolubilidad del grupo, ya que los tamaños de clase de los elementos en un $p$-complemento no dividen necesariamente a los tamaños de clase de dichos elementos en el propio grupo. En el año 2003, A. Beltrán y M.J. Felipe obtienen una generalización del teorema de Itô, considerando sólo tamaños de clase de conjugación de elementos $p$-regulares, para un cierto primo $p$, bajo la hipótesis de $p$-resolubilidad del grupo.

En este capítulo se desarrolla una demostración conjunta de los resultados obtenidos en [7] y [2], que establece que si $G$ es un grupo finito con tamaños de clase de conjugación de elementos $p$-regulares 1 y $m$, para algún entero $m$, entonces $m=p^{a} q^{b}$, con $p$ un primo distinto de $q$, y $a, b \geq 0$. Se demuestra que si $b=0$ entonces el grupo $G$ tiene $p$-complemento abeliano, y si $b \neq 0$ entonces $G=P Q \times A$, con $P \in \operatorname{Syl}_{p}(G), Q \in \operatorname{Syl}_{q}(G)$ y $A \leq \mathbf{Z}(G)$. Asímismo, se concluye que si $a=0$ entonces $G=P \times Q \times A$, con $A \leq \mathbf{Z}(G)$. En particular, se demuestra que $G$ es resoluble, obteniéndose una forma alternativa de probar el resultado de A. R. Camina sobre la resolubilidad de los grupos con dos tamaños de clases de $p^{\prime}$-elementos, que no hace uso de la Clasificación de Grupos Simples ó de resultados profundos de la Teoría de Grupos Finitos. 
Es importante señalar que en [7], Beltrán y Felipe se encuentran con dos situaciones distintas. Una situación en la que los centralizadores de los elementos $p$ regulares no centrales no son todos conjugados, análoga al caso ordinario, y otra situación, en la que los centralizadores de los elementos p-regulares no centrales sí que son todos conjugados. Esta es una situación nueva, de mayor complejidad, que no puede darse en el caso ordinario, ya que en tal circunstancia $G$ se obtiene como unión de conjugados de centralizadores, concluyéndose que el grupo es abeliano. Sin embargo, pueden encontrarse ejemplos de grupos en los que todos los centralizadores de elementos $p$-regulares no centrales son conjugados, como veremos en el tercer capítulo. Este es un claro ejemplo de las dificultades que surgen al trabajar con los elementos $p$-regulares del grupo, siendo necesario introducir técnicas nuevas para tratar los problemas que se plantean debido a que las extensiones de los resultados no son directas.

En el cuarto capítulo se investiga la estructura de un grupo a partir de los tamaños de clases de conjugación de sus elementos de orden potencia de primo o elementos primarios y se presentan los principales resultados que hemos obtenido y que aparecen recogidos en [4]. El hecho de que los tamaños de clases de los elementos compuestos sean múltiplos de los tamaños de clases de los elementos primarios sugiere que parte de la información estructural del grupo puede quedar codificada en el subconjunto de los tamaños de clase de sus elementos primarios, como se muestra en el capítulo. En 1996, L. Shirong prueba que si el grupo finito $G$ tiene exactamente dos tamaños de clases de elementos de orden potencia de primo, entonces es resoluble. Su demostración se apoya en la Clasificación de Grupos Simples y en el Teorema de Feit-Thompson sobre la resolubilidad de los grupos de orden impar, lo que demuestra la dificultad que surge al trabajar sólo con tamaños de clase de elementos primarios (elementos de orden potencia de primo), dado que la prueba del teorema de Itô es sencilla. Siguiendo un esquema similar al resultado de Shirong, X. Zhao y X. Guo prueban recientemente, bajo la hipótesis de $p$-resolubilidad del grupo, que si $G$ es $p$-resoluble con dos tamaños de clases de $p^{\prime}$-elementos de orden potencia de primo, entonces $G$ es resoluble. En el resultado principal del cuarto capítulo se demuestra que este tipo de grupos tiene la misma estructura de grupo que la obtenida en el tercer capítulo para todas las clases de $p^{\prime}$-elementos; es decir, $G$ tiene $p$-complemento abeliano o $G$, salvo factores centrales es $\{p, q\}$-grupo, con $p$ y $q$ dos primos distintos. Obtenemos por tanto una nueva generalización del resultado dado por Beltrán y Felipe en [7], bajo la hipótesis de p-resolubilidad del grupo.

En particular, si los tamaños de clase de elementos de $G$ de orden potencia de primo son 1 y $m$, para algún entero $m$, se aplica el resultado de resolubilidad de Shirong y se obtiene que $m$ es potencia de un primo $p$ y que $G$ es producto directo de un $p$-grupo por un subgrupo abeliano. Por tanto, el grupo es nilpotente y se ob- 
tiene una nueva generalización del teorema de Itô para elementos de orden potencia de primo. Obsérvese que este es, probablemente, el conjunto más reducido que se puede considerar, ya que si debilitamos las hipótesis del teorema y trabajamos sólo con los elementos de orden primo, podemos encontrar ejemplos que muestran que la tesis del teorema de Itô es falsa.

También queremos hacer notar que ambos resultados principales se demuestran con métodos sencillos, a partir de la hipótesis de resolubilidad del grupo, que aplicamos teniendo en cuenta los resultados de Shirong y de Zhao y Guo. Insistimos de nuevo en la dificultad que surge al considerar sólo los tamaños de clases de $p^{\prime}$ elementos primarios.

En el quinto y último capítulo se estudia la estructura de los subgrupos normales de un grupo $G$ a partir de los tamaños de las $G$-clases de conjugación contenidas en el normal. Este es un tema poco investigado y la bibliografía es escasa en resultados. Además, la extensión de propiedades a subgrupos normales del grupo no son inmediatas, debido a que el conjunto de tamaños de clases del subgrupo normal y el conjunto de tamaños de $G$-clases contenidas en él, no tienen necesariamente el mismo cardinal. En particular se demuestra que si $N$ es un subgrupo normal de $G$ tal que los tamaños de $G$-clases de $N$ son 1 y $m$, para algún entero $m$, entonces $N$ es abeliano ó es producto directo de un p-grupo no abeliano por un subgrupo central de $G$, y por tanto, es nilpotente. La conclusión final se obtiene demostrando primero la nilpotencia para grupos resolubles, y extendiendo el resultado al caso no resoluble, para lo cual nos servimos del conocimiento de la estructura de los CPgrupos, o grupos con todos sus elementos de orden potencia de primo, determinada recientemente por H. Heineken para el caso no resoluble.

Concluimos el capítulo extendiendo una conocida propiedad de los $p$-grupos demostrada por varios autores independientemente, que establece que para un $p$-grupo $G$ con dos tamaños de clases de conjugación, el grupo cociente $G / \mathbf{Z}(G)$ tiene exponente $p$. Concretamente demostramos que si $G$ es un grupo finito y $P$ es un p-subgrupo normal no abeliano de $G$, con dos tamaños de $G$-clases de conjugación, entonces $P /(\mathbf{Z}(G) \cap P)$, y en particular $P / \mathbf{Z}(P)$, tienen exponente $p$. Todos estos resultados aparecen publicados en [3]. 


\section{Capítulo 1}

\section{Tamaños de clases de conjugación y estructura de grupos}

Con el fin de obtener un mayor conocimiento sobre la estructura de los grupos, algunos autores se han interesado por determinar qué información puede extraerse de los tamaños de clase de conjugación de todos o parte de los elementos del grupo, cuando estos tamaños de clase verifican determinadas condiciones. Recordemos que el tamaño de clase de un elemento del grupo, que también se conoce como índice del elemento, coincide con el número de coclases de su centralizador en el grupo.

Así por ejemplo, varios autores han estudiado la estructura de un grupo cuando todos o parte de sus elementos tienen tamaño de clase potencia de primo. Los primeros resultados son los de M.L. Sylow ([63]) y W. Burnside ([20]). Por un lado, Sylow demuestra que un grupo cuyos índices son todos potencia de un primo dado tiene centro no trivial, y por otro, Burnside prueba que si $G$ tiene un elemento $x$ con tamaño de clase de conjugación potencia de un primo, entonces es no simple. Posteriormente en 1990, S.L. Kazarin ([46]) obtiene una extensión de este resultado demostrando que en tales circunstancias el grupo $\left\langle x^{G}\right\rangle$ generado por los elementos conjugados de $x$ es subgrupo normal resoluble de $G$. Utilizando este resultado, A.R. Camina y R.D. Camina ([23]) muestran que cualquier elemento con índice potencia de primo está en el segundo Fitting. A.R. Camina también prueba en [21], que si $G$ es un grupo finito y $p^{a}$ es la mayor potencia del primo $p$ que divide a un tamaño de clase en $G$ entonces, si existe un $p$-elemento en $G$ con índice $p^{a}$, el grupo posee p-complemento normal.

Considerando aquellos primos que dividen el orden del grupo y no dividen a ningún tamaño de clase en $G$, podemos encontrar algunos resultados elementales, por ejemplo, que un grupo $G$ tiene un $p$-subgrupo de Sylow central si y sólo si el primo $p$ divide a $|G|$ y no divide a ningún elemento de $\operatorname{cs}(G)$, donde $\operatorname{cs}(G)$ denota 
el conjunto de tamaños de clase de conjugación de $G$. También obtenemos otros resultados más profundos y difíciles, como el de D. Chillag y M. Herzog ([26]) de 1990, que demuestran que si ningún elemento de $c s(G)$ es divisible por 4, entonces $G$ es resoluble.

La información que podemos obtener sobre la estructura de un grupo depende del conjunto de sus tamaños de clase. Así por ejemplo, sabemos que $G$ es abeliano si y sólo si $G$ tiene un único tamaño de clase, es decir $\operatorname{cs}(G)=\{1\}$, pero no sabemos de qué grupo abeliano se trata. ¿Qué información se conoce cuando el grupo tiene dos tamaños de clase?

N. Itô demostró en $1953([43])$ que si $c s(G)=\{1, m\}$, entonces $m=p^{a}$, para algún primo $p$, y $G$ es nilpotente. En particular, $G=P \times A$ con $P \in \operatorname{Syl}_{p}(G)$ y $A$ un $p^{\prime}$-grupo abeliano, y además $P$ tiene un subgrupo normal abeliano $H$ tal que $P / H$ tiene exponente $p$. Este resultado, que permite enfocar el estudio de la estructura de los grupos con dos tamaños de clase de conjugación a través del conocimiento de los p-grupos, motivó la obtención y aplicación de algunos resultados importantes como los que se mencionan a continuación.

En 1951, Knoche demostró ([48]) que $G$ es un p-grupo con tamaños de clase 1 y $p$ si y sólo si el orden del grupo derivado es $p$, mientras que en 1953, Itô prueba que si los tamaños de clase de un $p$-grupo son 1 y $p^{a}$ entonces $G$ tiene un subgrupo normal abeliano $A$ tal que $G / A$ tiene exponente $p$. En particular, si $p=2$ entonces $G$ es metabeliano. En 1970, Isaacs demostró que si los tamaños de clase del $p$-grupo son 1 y $p^{a}$ entonces el grupo cociente $G / \mathbf{Z}(G)$ tiene exponente $p$, y en el año 2002, Ishikawa demuestra ([42]) que para estos grupos la clase de nilpotencia del grupo es menor o igual que 3 y el grupo derivado es elemental abeliano.

Debemos citar el resultado de E. Fisman y Z. Arad ([5]) del año 1987, que prueban que si $G$ tiene dos tamaños de clase coprimos, entonces es no simple. En su demostración utilizan la Clasificación de Grupos Simples. Asímismo, en 1996, L. Shirong ([62]) obtiene una extensión del citado teorema de Itô ([43]), probando la resolubilidad de los grupos finitos cuyos elementos de orden potencia de primo tienen tamaños de clase 1 ó $m$.

El estudio de la estructura de los grupos finitos con tres tamaños de clase de conjugación tiene su principal resultado en un teorema de Itô de 1970 ([44]) que dice que si $\operatorname{cs}(G)=\{1, m, n\}$, entonces $G$ es resoluble. En su demostración, Itô utiliza el Teorema de Feit-Thompson y algunos resultados profundos de la Clasificación de Grupos Simples debidos a M. Suzuki. Poco después, en 1971, J.Rebmann ([57]) simplifica este resultado para el caso de $F$-grupos, determinando la estrutura de este 
tipo de grupos. Recordemos que un grupo $G$ verifica la propiedad $F$, ó es $F$-grupo, si para cualquier par de elementos no centrales $x, y$ de $G$ se verifica que $\mathbf{C}_{G}(x) \not \leq \mathbf{C}_{G}(y)$ y $\mathbf{C}_{G}(y) \not \leq \mathbf{C}_{G}(x)$. Por su parte, S.L. Kazarin ([47]), y asimismo Bertram, Herzog y Mann ([17]), prueban independientemente que si $c s(G)=\{1, m, n\}$, donde $m$ y $n$ son enteros coprimos, entonces $G / \mathbf{Z}(G)$ es grupo Frobenius.

En 1971, A. R. Camina ([22]) utiliza la descripción de los grupos finitos con 2-subgrupos de Sylow diédricos, dada por D. Gorenstein y J.H. Walter, para demostrar que si $G$ es un grupo con tres tamaños de clase que no es $F$-grupo, entonces es producto directo de un grupo abeliano por un $\{p, q\}$-grupo. Con este resultado y el de Rebmann se obtiene la resolubilidad de los grupos con tres tamaños de clases de conjugación, dada inicialmente por Itô. Por otro lado, Camina analiza en [21] la estructura de los grupos con $c s(G)=\left\{1, p^{a}, p^{a} q^{b}\right\}$. Este análisis ha sido extendido por A. Beltrán y M.J. Felipe en [15] para grupos con $c s(G)=\{1, m, m n\}$, con $m$ y $n$ dos enteros coprimos, que demuestran que $m=p$ y obtienen algunas propiedades estructurales de los $p$-subgrupos de Sylow y los $p$-complementos.

Recientemente, S. Dolfi y E. Jabara ([29]) mejoran los resultados anteriores, caracterizando los grupos con tres tamaños de clases de conjugación, pero en su demostración utilizan la resolubilidad de este tipo de grupos debida a Itô. Estos autores demuestran que dichos grupos son $F$-grupos o son producto directo de un grupo abeliano por un grupo de orden potencia de primo, y después aplican el resultado de Rebmann para clasificar los $F$-grupos con tres tamaños de clase.

El estudio de los grupos con cuatro tamaños de clase de conjugación nos lleva al año 1970, en que Itô ([44]) demuestra que los únicos grupos simples con $|\operatorname{cs}(G)|=4$ son los grupos $S L\left(2,2^{m}\right)$, con $m \geq 2$. Poco después, en 1972 Camina ([21]) prueba que si $\operatorname{cs}(G)=\left\{1, p^{a}, q^{b}, p^{a} q^{b}\right\}$, entonces $G$ es nilpotente.

En el año 2006, A. Beltrán y M.J. Felipe extienden ([10], [11]) el resultado de Camina a grupos con $c s(G)=\{1, m, n, m n\}$, siendo $m$ y $n$ coprimos, y prueban que para estos grupos $n=p^{a}$ y $m=q^{b}$, con $p$ un primo distinto de $q$, y $G$ es nilpotente. Los autores generalizan ([14]) poco después este resultado para grupos resolubles con $c s(G)=\{1, m, n, m k\}$, con $m$ y $n$ coprimos y $k$ un divisor estricto de $n$, concluyendo que si $\pi=\pi(m)$, entonces $k=q^{a}$ para un cierto primo $q, G$ posee un $\pi$-subgrupo de Hall abeliano y los $\pi$-complementos de $G$ son de la forma $Q A$, con $Q \in \operatorname{Syl}_{q}(G)$ y $A$ abeliano. Más recientemente, Beltrán y Felipe han clasificado ([16]), utilizando técnicas sencillas, los grupos con cuatro tamaños de clase tales que dos de ellos son coprimos. Por otro lado, A.R. Camina y R.D. Camina prueban en [24] que cuando un grupo tiene más de tres tamaños de clase y de cada tres, dos de ellos son coprimos, entonces el grupo tiene como máximo cuatro tamaños de clase 
y es resoluble. En su demostración utilizan la Clasificación de Grupos Simples.

Algunos autores han estudiado los grupos cuyos tamaños de clase son todos libres de cuadrados. Por ejemplo, J. Cossey y Y. Wang demuestran ([28]) que estos grupos son superresolubles y que ambos, $G / \mathbf{F}(G)$ y $G^{\prime}$ son grupos cíclicos de orden libre de cuadrados. Además concluyen que la clase del subgrupo Fitting de $G$ es como mucho 2 y que $G$ es metabeliano. Utilizando los resultados en [31], Shirong refuerza este resultado demostrando ([61]) que si $p$ es el primo más pequeño que divide el orden del grupo $G$, y asumiendo que $p^{2}$ no divide el tamaño de clase de ningún elemento de orden potencia de $q$, para un primo $q$ distinto de $p$, entonces $G$ es $p$-nilpotente, y en particular, resoluble.

Como se ha comentado en la introducción, nuevas líneas de investigación aparecen en el estudio de las clases de conjugación de elementos reales y, en particular, de elementos racionales. Por citar algunos de los resultados obtenidos, en 1979, S. Iwasaki ([45]) caracteriza los grupos que tienen exactamente dos clases de conjugación reales. Posteriormente, en [52], Moretó y Navarro extienden los resultados de Iwasaki y analizan la estructura de los grupos con tres clases reales. Por otro lado, D. Chillag y A. Mann caracterizan ([27]) aquellos grupos tales que todo elemento racional es central. Este resultado ha sido posteriormente generalizado por G. Navarro y L. Sanus en [53] para clases racionales. Por su parte, S. Dolfi, E. Pacifici y L. Sanus determinan en [30] la estructura de los grupos que tienen todas las clases reales no centrales de tamaño un primo, probando que el grupo es resoluble ([30]). Recientemente J. Sangroniz y J. Tent determinan la estructura de los 2-grupos finitos con 4 ó 5 clases racionales.

Por otro lado, se han obtenido algunos resultados sobre la estructura del grupo cuando los tamaños de clase de conjugación de sus elementos $p$-regulares verifican determinadas condiciones aritméticas. Estos resultados, que extienden o generalizan propiedades conocidas en el caso ordinario al caso $p$-regular, no son siempre directas y ello se debe, principalmente, al hecho de que los tamaños de clase en un $p$-complemento no necesariamente dividen a los tamaños de clase en el grupo. Por otro lado, las demostraciones de algunos de estos resultados para clases $p$-regulares introducen pruebas alternativas más sencillas que sus análogas para el caso ordinario. Si además, el grupo no es $p$-resoluble, las extensiones o generalizaciones se complican, al no poder garantizarse la existencia de $p$-complementos.

El interés por el estudio de los tamaños de clases de conjugación de los elementos $p$-regulares y su influencia en la $p$-estructura del grupo es reciente. Durante los años 90 aparecen algunos resultados, entre los que podemos citar el trabajo de Y.C. Ren ([56]) o los trabajos de Y. Ninomiya ([54],[55]). Ren determina la estructura de los 
grupos con valores de $r c_{q}(G)$ pequeños, siendo $q$ el menor primo que divide a $|G|$ y $r c_{q}(G)$ la mayor potencia de $q$ que divide a los cardinales de las clases $p$-regulares de $G$.

En 1972, Camina demuestra ([21]) que si $G$ es un grupo y $p$ un primo tal que todo elemento de $c s_{p^{\prime}}(G)$ es $p^{\prime}$-número entonces $G=P \times H$, con $P \in \operatorname{Syl}_{p}(G)$ y $H$ un $p$-complemento de $G$, donde $c s_{p^{\prime}}(G)$ denota el conjunto de tamaños de clase de conjugación de $G_{p^{\prime}}$, siendo $G_{p^{\prime}}$ el conjunto de $p^{\prime}$-elementos de $G$. Posteriormente $\mathrm{X}$. Liu, Y. Wang y H. Wei obtienen una variante debilitada de este resultado ([50]) que dice que si $p$ es un primo que no divide ningún tamaño de clase de elementos de orden potencia de primo en $G_{p^{\prime}}$, entonces el $p$-subgrupo de Sylow de $G$ es un factor directo de $G$.

En el año 2001, S. Dolfi y M.S. Lucido introducen la siguiente definición: un grupo finito $G$ verifica la propiedad $P(p, q)$ si cada $p^{\prime}$-elemento tiene $q^{\prime}$-índice. Estos autores demuestran que si $G$ es un grupo que satisface la propiedad $P(p, q)$, con $p$ un primo distinto de $q$, entonces $\mathbf{O}^{p}(G)$ es $q$-nilpotente y $G$ tiene $q$-subgrupos de Sylow abelianos. También obtienen una clasificación para este tipo de grupos utilizando la Clasificación de Grupos Simples. Posteriormente S. Dolfi, A. Moretó y G. Navarro determinan la estructura de los grupos que tienen exactamente una clase de conjugación con tamaño un múltiplo de un primo $p$.

Por otra parte, Beltrán y Felipe obtienen en [7] una caracterización de los grupos con $p$-complementos abelianos demostrando que todo tamaño de clase en $G_{p^{\prime}}$ es potencia de un cierto primo $p$ si y sólo si $G$ tiene $p$-complementos abelianos. También obtienen una extensión del teorma de Itô ([43]) para grupos $p$-resolubles con $c s_{p^{\prime}}(G)=\{1, m\}$. Concretamente, los autores prueban que $m=p^{a}$ y $G$ tiene $p$-complemento abeliano ó $m=p^{a} q^{b}$, con $p$ y $q$ primos distintos, y $G=P Q \times A$, con $P \in \operatorname{Syl}_{p}(G), Q \in \operatorname{Syl}_{q}(G)$ y $A \in \mathbf{Z}(G)$.

Otro resultado sobre grupos con $c s_{p^{\prime}}(G)=\{1, m\}$ es el de Camina de 1974 ([22]), que demuestra que si $G$ es un grupo finito con todas las clases de conjugación de elementos $p$-regulares no centrales del mismo tamaño y $|G / \mathbf{Z}(G)|$ es divisible al menos por dos primos distintos de $p$, entonces $G$ es resoluble. En [2], E. Alemany, A. Beltrán y M.J. Felipe presentan una demostración alternativa más sencilla de este resultado y la utilizan para eliminar la condición de $p$-resolubilidad en la hipótesis del teorema principal de [7], anteriormente citado. Obtienen así una prueba unificada de los dos resultados.

Por su parte, A.R. Camina y R.D. Camina demuestran en un estudio reciente sobre la influencia de los tamaños de clases de conjugación sobre la estructura de los 
grupos finitos que si $G$ es un grupo resoluble y $p$ un primo, asumiendo que ningún tamaño de clase en $G_{p^{\prime}}$ es divisible por $p^{2}$, entonces $G / \mathbf{O}_{p^{\prime}}(G)$ es un $\pi^{\prime}$-grupo, siendo $\pi$ el conjunto de primos divisores de $|G|$, distintos de $p$, que no dividen a $p-1$.

En [8] Beltrán y Felipe extienden el resultado principal de M. Bianchi et al. ([18]) para clases ordinarias y demuestran que si $G$ es un grupo $p$-resoluble y $m$ y $n$ son dos elementos maximales de $c s_{p^{\prime}}(G)$, con $m$ y $n$ coprimos, $m, n>1$, y $p$ es un primo que no divide a $m$, entonces $G$ es resoluble, $c s_{p^{\prime}}(G)=\{1, m, n\}$ y cada $p$-complemento de $G$ es un grupo cuasi-Frobenius con núcleo y complemento abelianos. También se demuestra que los tamaños de clase del $p$-complemento son $\left\{1, m_{p^{\prime}}, n_{p^{\prime}}\right\}$. Como corolario de este teorema se obtiene que si $G$ es un grupo $p$ resoluble y $c s_{p^{\prime}}(G)=\{1, m, n\}$, con $m$ y $n$ coprimos y $m, n>1$, entonces $G$ es resoluble y los $p$-complementos de $G$ son cuasi-Frobenius con núcleo y complementos abelianos. Recordemos que un grupo $G$ se dice que es cuasi-Frobenius si $G / \mathbf{Z}(G)$ es Frobenius. En tal caso, la imagen inversa en $G$ del núcleo y de un complemento se conocen como núcleo y complemento de $G$.

Aplicando este último resultado, Beltrán y Felipe obtienen en [8] la estructura de los grupos $p$-resolubles cuyos tamaños de clase $p$-regulares no centrales son números enteros consecutivos, extendiendo así el resultado obtenido por Bianchi et al. ([19]) para clases de conjugación ordinarias. En este trabajo también se obtiene una caracterización de los grupos $p$-resolubles cuyas clases de conjugación $p$-regulares tienen cardinal potencia de primo, lo que constituye una extensión de los dos resultados de D. Chillag y M. Herzog para clases de conjugación ordinarias recogidos en [26].

Un resultado análogo al obtenido por Beltrán y Felipe en [14], comentado anteriormente, para grupos $p$-resolubles con condiciones similares en clases de conjugación $p$-regulares, es el obtenido por estos autores en [12] donde demuestran que si $G$ es un grupo $p$-resoluble con $c s_{p^{\prime}}(G)=\{1, m, n, m n\}$, siendo $m$ y $n$ enteros positivos coprimos, y $p$ no divide a $m$ y $n$, o $n=p^{a}$, entonces los $p$-complementos de $G$ son nilpotentes y $m$ y $n$ son potencias de algún primo. Queda abierto el problema del estudio de la estructura cuando del grupo cuando $m$ y $n$ no son potencia de primo.

Otra línea de investigación poco explorada enfoca el estudio de la estructura del grupo a partir de los tamaños de clase de sus elementos de orden potencia de primo. El primer resultado se debe a R. Baer ([6]) que demuestra en el año 1953 que un grupo $G$, cuyos tamaños de clase de elementos de orden potencia de primo son también potencia de primo, es resoluble. Posteriormente en el año 1996, L. Shirong utiliza la Clasificación de Grupos Simples y el teorema de Feit-Thompson para demostrar ([62]) la resolubilidad de los grupos con dos tamaños de clase de elementos de orden potencia de primo. En [4], Alemany, Beltrán y Felipe obtienen la nilpoten- 
cia de este tipo de grupos y extienden la demostración al caso no resoluble haciendo uso del resultado de Shirong. Recientemente X. Zhao y X. Guo ([66]) generalizan el resultado de Shirong en el contexto de $p^{\prime}$-elementos. Concretamente prueban que si $G$ es un grupo $p$-resoluble con dos tamaños de clase de conjugación de $p^{\prime}$-elementos de orden potencia de primo, entonces $G$ es resoluble. En [4], Alemany, Beltrán y Felipe prueban que en realidad $G$ es nilpotente. Concretamente prueban que $G$ tiene p-complemento abeliano o $G=P Q \times A$, obteniéndose una nueva generalización del caso ordinario, anteriormente mencionado.

Finalmente, hemos de comentar que recientemente se han obtenido algunos resultados que relacionan la estructura del grupo y de sus subgrupos normales con los tamaños de las $G$-clases de conjugación de los elementos del normal. Este tipo de información parcial relativa a la estructura normal de un grupo a partir de sus tamaños de $G$-clases es un área novedosa. En [34], X. Zhao y X. Guo hacen un esfuerzo en esta dirección probando que si $N$ es un subgrupo normal de $G$ con dos tamaños de $G$-clases y $N$ contiene un $r$-subgrupo de Sylow no central de $G$, para un cierto primo $r$, entonces $N$ es nilpotente. En [3], Alemany, Beltrán y Felipe mejoran este resultado, eliminando la condición de existencia del $r$-subgrupo de Sylow, y demuestran que $N$ es nilpotente. En particular demuestran que $N$ es abeliano, o es producto directo de un $p$-grupo por un subgrupo central de $G$. Asímismo, los autores extienden una conocida propiedad de los $p$-grupos con dos tamaños de clases ordinarios y demuestran que si $P$ es un $p$-subgrupo normal de un grupo $G$ con dos tamaños de $G$-clases de conjugación, entonces el grupo cociente $P / \mathbf{Z}(P)$ tiene exponente $p$.

Respecto a grupos que poseen un subgrupo normal con tres tamaños de $G$-clases, cabe destacar el reciente resultado obtenido por Z. Akhalaghi et al. en [1], que establece la resolubilidad de un subgrupo normal $N$ de un grupo $G$, cuando el conjunto de los tamaños de las $G$-clases es $c s_{G}(N)=\{1, m, n\}$, con $m<n$ y $m$ no divide a $n$. Los autores obtienen además algunas propiedades estructurales del subgrupo normal, dejando como problema abierto el caso en que $m$ divide a $n$. 



\section{Capítulo 2}

\section{Resultados previos}

En los capitulos siguientes, al abordar nuestro estudio sobre ciertas propiedades estructurales de un grupo finito a partir de sus tamaños de clases de conjugación, hemos utilizado algunos resultados que recopilamos en este capítulo. Omitimos la demostración de algunos de ellos, por ser resultados estándar en Teoría General de Grupos.

Para trabajar con elementos $p$-regulares o de orden potencia de primo, y con sus clases de conjugación, es imprescindible conocer la $\left\{p, p^{\prime}\right\}$-factorización de un elemento cualquiera del grupo. Esta factorización puede extenderse para obtener la descomposición de un elemento como producto de elementos de orden potencia de primo.

Lema 2.1 Sea $G$ un grupo finito, $g \in G$ y $p$ un número primo que divide $|G|$. Entonces existen dos elementos únicos, $g_{p}$ y $g_{p^{\prime}}$ tales que:

1. $g_{p}$ es un elemento potencia de $g$ y de orden potencia de $p$.

2. $g_{p^{\prime}}$ es un elemento potencia de $g$ y de orden no divisible por $p$.

3. $g=g_{p} g_{p^{\prime}}=g_{p^{\prime}} g_{p}$.

$\mathrm{Al}$ elemento $g_{p}$ se le conoce como la $p$-parte de $g$ y al elemento $g_{p^{\prime}}$ como la $p^{\prime}$-parte de $g$. Obsérvese que, al ser $g_{p}$ y $g_{p^{\prime}}$ potencias de $g$, se tiene que $\mathbf{C}_{G}(g) \subseteq \mathbf{C}_{G}\left(g_{p}\right)$ y $\mathbf{C}_{G}(g) \subseteq \mathbf{C}_{G}\left(g_{p^{\prime}}\right)$.

Considerando un conjunto arbitrario de primos y la unicidad de la descomposición del lema anterior se obtiene el siguiente resultado.

Corolario 2.2 Sea $G$ un grupo finito, $g \in G$ y $\pi$ un conjunto de primos. Entonces existen dos elementos únicos, $g_{\pi}$ y $g_{\pi^{\prime}}$ tales que: 
1. Todo primo divisor de $o\left(g_{\pi}\right)$ pertenece al conjunto $\pi$ y $g_{\pi}$ es potencia del elemento $g$.

2. Los primos divisores de $o\left(g_{\pi^{\prime}}\right)$ no pertenecen al conjunto $\pi$ y $g_{\pi^{\prime}}$ es potencia del elemento $g$.

3. $g=g_{\pi} g_{\pi^{\prime}}=g_{\pi^{\prime}} g_{\pi}$.

Al elemento $g_{\pi}$ se le conoce como la $\pi$-parte de $g$ y al elemento $g_{\pi^{\prime}}$ como la $\pi^{\prime}$-parte de $g$. De nuevo, al ser $g_{\pi}$ y $g_{\pi^{\prime}}$ potencias de $g$, se tiene que $\mathbf{C}_{G}(g) \subseteq \mathbf{C}_{G}\left(g_{\pi}\right)$ y $\mathbf{C}_{G}(g) \subseteq \mathbf{C}_{G}\left(g_{g_{\pi^{\prime}}}\right)$.

La siguiente propiedad general de los conmutadores de los elementos de un grupo nos facilitará la manipulación algebraica de los mismos en resultados posteriores. Obsérvese que el lema es aplicable cuando el conmutador de dos elementos es central.

Lema 2.3 Consideremos un grupo $G$ y dos elementos $x, y \in G$ tales que $[x, y] \in$ $\mathbf{C}_{G}(x) \cap \mathbf{C}_{G}(y)$. Entonces $\left[x^{n}, y\right]=[x, y]^{n}=\left[x, y^{n}\right]$, para todo $n \in \mathbb{Z}$.

Demostración. Procederemos por inducción sobre $n$. Recordemos la siguiente propiedad de los conmutadores

a) $[x, y z]=[x, z][x, y]^{z}$

b) $[x z, y]=[x, y]^{z}[z, y]$

Si $n=0$ ó $n=1$, el resultado se obtiene trivialmente. Si $n=2$, se tiene que

$$
\left[x^{2}, y\right]=[x, y]^{x}[x, y]=[x, y][x, y]=[x, y]^{2}
$$

y de manera análoga

$$
\left[x, y^{2}\right]=[x, y][x, y]^{y}=[x, y][x, y]=[x, y]^{2}
$$

Supongamos primero $n>0$. Por la hipótesis de inducción se tiene que $\left[x^{n-1}, y\right]=$ $[x, y]^{n-1}=\left[x, y^{n-1}\right]$. Entonces

$$
\left[x^{n}, y\right]=\left[x^{n-1} x, y\right]=\left[x^{n-1}, y\right]^{x}[x, y]=\left([x, y]^{n-1}\right)^{x}[x, y]=[x, y]^{n-1}[x, y]=[x, y]^{n}
$$

y, asimismo,

$$
\left[x, y^{n}\right]=\left[x, y^{n-1} y\right]=[x, y]\left[x, y^{n-1}\right]^{y}=[x, y]\left([x, y]^{n-1}\right)^{y}=[x, y][x, y]^{n-1}=[x, y]^{n}
$$


Supongamos ahora que $n<0$. Entonces

$$
1=\left[x^{-n} x^{n}, y\right]=\left[x^{-n}, y\right]^{x^{n}}\left[x^{n}, y\right]=\left([x, y]^{-n^{x^{n}}}\right)\left[x^{n}, y\right]=[x, y]^{-n}\left[x^{n}, y\right]
$$

de manera que $\left[x^{n}, y\right]=[x, y]^{n}$. Del mismo modo,

$$
1=\left[x, y^{-n} y^{n}\right]=\left[x, y^{n}\right]\left[x, y^{-n}\right]^{y^{n}}=\left[x, y^{n}\right]\left([x, y]^{-n y^{n}}\right)=\left[x, y^{n}\right][x, y]^{-n},
$$

y se obtiene que $\left[x, y^{n}\right]=[x, y]^{n}$.

El siguiente lema recoge algunos resultados que utilizaremos frecuentemente en capítulos posteriores y que relacionan los tamaños de clases de los elementos de un subgrupo normal del grupo con sus correspondientes tamaños de clases en el grupo. También muestra la relación entre los centralizadores de elementos del grupo de orden coprimo que conmutan. Recordemos que $x^{G}=\left\{g^{-1} x g: g \in G\right\}$ denota la clase de conjugación de un elemento $x$ de $G$.

Lema 2.4 Sea $G$ un grupo finito, $N \unlhd G, x \in N$ y $g \in G$. Entonces:

1. $\left|x^{N}\right|$ divide $a\left|x^{G}\right|$.

2. $\left|g N^{G / N}\right|$ divide $a\left|g^{G}\right|$.

3. Si $x, y \in G$ son de orden coprimo y conmutan, entonces $\mathbf{C}_{G}(x y)=\mathbf{C}_{G}(x) \cap$ $\mathbf{C}_{G}(y)$

Demostración. (1) Como $N \unlhd G$ y $\mathbf{C}_{G}(x) \leq G$ se tiene que $N \mathbf{C}_{G}(x) \leq G$. Aplicando el Teorema de Lagrange sobre la transitividad de índices se tiene que

$$
\left|G: \mathbf{C}_{N}(x)\right|=\left|G: \mathbf{C}_{G}(x)\right|\left|\mathbf{C}_{G}(x): \mathbf{C}_{N}(x)\right|
$$

Como además $\mathbf{C}_{N}(x) \leq N \leq N \mathbf{C}_{G}(x) \leq G$, aplicando de nuevo la transitividad de índices

$$
\left|G: \mathbf{C}_{N}(x)\right|=\left|G: N \mathbf{C}_{G}(x)\right|\left|N \mathbf{C}_{G}(x): N\right|\left|N: \mathbf{C}_{N}(x)\right|,
$$

y por el segundo Teorema de Isomorfía de grupos

$$
N \mathbf{C}_{G}(x) / N \cong \mathbf{C}_{G}(x) /\left(\mathbf{C}_{G}(x) \cap N\right)
$$

por lo que,

$$
\left|N \mathbf{C}_{G}(x) / N\right|=\left|\mathbf{C}_{G}(x) /\left(\mathbf{C}_{G}(x) \cap N\right)\right| .
$$


Es decir, $\left|N \mathbf{C}_{G}(x): N\right|=\left|\mathbf{C}_{G}(x): \mathbf{C}_{G}(x) \cap N\right|=\left|\mathbf{C}_{G}(x): \mathbf{C}_{N}(x)\right|$, y sustituyendo este resultado en la ecuación 2.1 , se tiene

$$
\left|G: \mathbf{C}_{N}(x)\right|=\left|G: N \mathbf{C}_{G}(x)\right|\left|\mathbf{C}_{G}(x): \mathbf{C}_{N}(x)\right|\left|N: \mathbf{C}_{N}(x)\right| .
$$

De manera análoga, $\left|G: \mathbf{C}_{N}(x)\right|=\left|G: \mathbf{C}_{G}(x)\right|\left|\mathbf{C}_{G}(x): \mathbf{C}_{N}(x)\right|$, de donde

$$
\left|G: \mathbf{C}_{G}(x)\right|=\left|G: \mathbf{C}_{N}(x)\right| /\left|\mathbf{C}_{G}(x): \mathbf{C}_{N}(x)\right|,
$$

Y sustituyendo ahora en la ecuación 2.2 y simplificando se obtiene que

$$
\left|G: \mathbf{C}_{G}(x)\right|=\left|G: N \mathbf{C}_{G}(x)\right|\left|N: \mathbf{C}_{N}(x)\right|
$$

y por tanto $\left|x^{N}\right|$ divide a $\left|x^{G}\right|$.

(2) Veamos en primer lugar que

$$
\mathbf{C}_{G}(g) N / N \leq \mathbf{C}_{G / N}(g N)
$$

Si $h N$ es un elemento de $\mathbf{C}_{G}(g) N / N$ entonces $h \in \mathbf{C}_{G}(g) N$ y, por tanto, existen $h_{1} \in \mathbf{C}_{G}(g)$ y $n_{1} \in N$ tales que $h=h_{1} n_{1}$. Es decir $h N=h_{1} n_{1} N=h_{1} N$. Como $(h N)(g N)=\left(h_{1} N\right)(g N)=\left(h_{1} g\right) N=\left(g h_{1}\right) N=(g N)\left(h_{1} N\right)=(g N)(h N)$, por ser $h_{1} \in \mathbf{C}_{G}(g)$, se tiene que $(h N) \in \mathbf{C}_{G / N}(g N)$.

Como $\mathbf{C}_{G}(g) N / N \leq \mathbf{C}_{G / N}(g N) \leq G / N$, aplicando el Teorema de transitividad de índices se obtiene que

$$
\left|G / N: \mathbf{C}_{G / N}(g N)\right|\left|\mathbf{C}_{G / N}(g N): \mathbf{C}_{G}(g) N / N\right|=\left|G / N: \mathbf{C}_{G}(g) N / N\right|,
$$

de manera que

$$
\frac{|G| /|N|}{\left|\mathbf{C}_{G}(g) N\right| /|N|}=\frac{|G|}{\left|\mathbf{C}_{G}(g) N\right|}=\left|G: \mathbf{C}_{G}(g) N\right|
$$

y como $\left|G: \mathbf{C}_{G}(g)\right|=\left|G: \mathbf{C}_{G}(g) N\right|\left|\mathbf{C}_{G}(g) N: \mathbf{C}_{G}(g)\right|$, despejando $\left|G: \mathbf{C}_{G}(g) N\right|$ y sustituyendo en la ecuación 3.1 , nos queda

$$
\left|G / N: \mathbf{C}_{G / N}(g N)\right|\left|\mathbf{C}_{G / N}(g N): \mathbf{C}_{G}(g) N / N\right|=\frac{\left|G: \mathbf{C}_{G}(g)\right|}{\left|\mathbf{C}_{G}(g) N: \mathbf{C}_{G}(g)\right|}
$$

Por tanto, 


$$
\begin{aligned}
\left|G: \mathbf{C}_{G}(g)\right| & =\left|\mathbf{C}_{G}(g) N: \mathbf{C}_{G}(g)\right|\left|G / N: \mathbf{C}_{G / N}(g N)\right|\left|\mathbf{C}_{G / N}(g N): \mathbf{C}_{G}(g) N / N\right| \\
& =\left|\mathbf{C}_{G}(g) N: \mathbf{C}_{G}(g)\right|\left|(g N)^{G / N}\right|\left|\mathbf{C}_{G / N}(g N): \mathbf{C}_{G}(g) N / N\right|
\end{aligned}
$$

y concluimos que $\left|g N^{G / N}\right|$ divide a $\left|g^{G}\right|$.

(3) Es fácil ver que $\mathbf{C}_{G}(x) \cap \mathbf{C}_{G}(y) \leq \mathbf{C}_{G}(x y)$. Demostraremos que el recíproco también se verifica.

Sea $g=x y$. Como $x$ e $y$ son de orden coprimo y conmutan, por el Corolario 2.2 se tiene que $x=g_{\pi}$ e $y=g_{\pi^{\prime}}$, para un cierto conjunto de primos $\pi$. Por tanto, $\mathbf{C}_{G}(g) \leq \mathbf{C}_{G}\left(g_{\pi}\right)$ y $\mathbf{C}_{G}(g) \leq \mathbf{C}_{G}\left(g_{\pi^{\prime}}\right)$. Es decir, $\mathbf{C}_{G}(x y) \leq \mathbf{C}_{G}(x) \cap \mathbf{C}_{G}(y)$.

La siguiente propiedad establece que un grupo finito no puede ser unión de conjugados de un subgrupo propio. Esta propiedad se utiliza mucho a lo largo de la memoria, por lo que obviaremos referenciarla.

Lema 2.5 Si $G$ es un grupo finito y $U \leq G$ es un subgrupo de $G$ tal que

$$
G=\bigcup_{g \in G} U^{g},
$$

entonces $G=U$.

Demostración. Véase 4.16 de [39].

El siguiente lema asocia la existencia de $p$-subgrupos de Sylow centrales en un grupo $G$ con el conjunto de primos divisores de $|G|$ que no dividen a ningún elemento de $\operatorname{cs}(G)$.

Lema 2.6 Sea $G$ un grupo finito y consideremos un cierto primo p. Entonces, $G$ tiene un p-subgrupo de Sylow $P$ tal que $P \subseteq \mathbf{Z}(G)$, para un cierto primo $p$, si y sólo si $p$ no divide $\left|x^{G}\right|$, para todo $x \in G$.

Demostración. Supongamos que $G$ tiene un $p$-subgrupo de Sylow $P$, para un cierto primo $p$, tal que $P \subseteq \mathbf{Z}(G)$. Entonces, para cada $x \in G$ se tiene que $|G|_{p}=\left|\mathbf{C}_{G}(x)\right|_{p}$, y por tanto, el primo $p$ no divide $\left|G: \mathbf{C}_{G}(x)\right|=\left|x^{G}\right|$.

Supongamos ahora que $p$ no divide a ningún elemento de $\operatorname{cs}(G)$. Trivialmente suponemos que $p$ divide a $|G|$. Para cada $g \in G$ existe $P \in \operatorname{Syl}_{p}(G)$ tal que $P \subseteq$ 
$\mathbf{C}_{G}(g)$. Por tanto $g \in \mathbf{C}_{G}(P)$ y $G=\bigcup_{g \in G} \mathbf{C}_{G}(P)^{g}$. Aplicando el Lema 2.5 se concluye que $G=\mathbf{C}_{G}(P)$.

El siguiente lema pone de manifiesto una propiedad fundamental del subgrupo Fitting $\mathbf{F}(G)$ de los grupos resolubles.

Lema 2.7 Sea $G$ es un grupo finito resoluble. Entonces $\mathbf{F}(G) \neq 1$ y

$$
\mathbf{C}_{G}(\mathbf{F}(G)) \subseteq \mathbf{F}(G) .
$$

Demostración. Véase 6.1.4 de [49].

En ocasiones, la acción coprima se puede utilizar para obtener una factorización del grupo o la estructura de determinados subgrupos del grupo. Supongamos que $A$ actúa como grupo de automorfismos de $G$. Se dice que $A$ actúa coprimamente sobre $G$ si $(|G|,|A|)=1$. En tal caso, si $S=A G$ es el producto semidirecto de $A$ con $G$, se tiene que $G \unlhd S$ y $A$ es un complemento de $G$ en $S$. En el siguiente teorema recogemos algunas de las propiedades de la acción coprima que utilizaremos en resultados posteriores.

Teorema 2.8 Sea $A$ un grupo que actúa sobre $G$.

a) Si $N$ es subgrupo normal $A$-invariante de $G$ y $(|N|,|A|)=1$, entonces $\mathbf{C}_{G / N}(A)=$ $\mathbf{C}_{G}(A) N / N$.

b) $S i(|G|,|A|)=1$ entonces $G=[G, A] \mathbf{C}_{G}(A)$.

c) (Lema de Fitting). Si $G$ es abeliano y $(|G|,|A|)=1$, entonces

$$
G=\mathbf{C}_{G}(A) \times[G, A] .
$$

Demostración. Véase 14.5 de [38].

Teorema 2.9 Supongamos que $H \leq A u t(G)$ actúa libre de puntos fijos, sobre $G$. Entonces:

1. Si $Q \in \operatorname{Syl}_{q}(H)$, entonces $Q$ es grupo cíclico o cuaternio generalizado. Si $P \in$ $\operatorname{Syl}_{p}(G)$ es invariante por $H$, entonces $H$ actúa libre de puntos fijos sobre $P / \Phi(P)$.

2. Si p y q son primos, entonces cada subgrupo de $H$ de orden pq es cíclico. 
Demostración. Véase Teorema 16.12 de [38].

El siguiente resultado es el conocido $P \times Q$-Lema de Thompson, que se obtiene como consecuencia de las propiedades de la acción coprima.

Teorema 2.10 Sea $P \times Q$ el producto directo de un p-grupo $P$ y un $p^{\prime}$-grupo $Q$ representado como grupo de automorfismos de un p-grupo $G$. Supongamos que $\boldsymbol{C}_{G}(P) \subseteq \boldsymbol{C}_{G}(Q)$. Entonces $Q$ actúa trivialmente sobre $G$.

Demostración. Véase 8.2.8 de [49].

Enunciamos finalmente algunos teoremas clásicos de la Teoría General de Grupos que hemos utilizado con cierta frecuencia. El primero de ellos es el famoso teorema de Kegel-Wielandt sobre la resolubilidad de un grupo que es producto de subgrupos nilpotentes.

Teorema 2.11 (Kegel - Wielandt). Si $G$ tiene subgrupos nilpotentes $G_{1}$ y $G_{2}$ tales que $G=G_{1} G_{2}$ entonces $G$ es resoluble.

Demostración. Véase VI.4.3 de [37].

Obsérvese que como consecuencia del teorema, si $G$ tiene subgrupos nilpotentes $G_{1}$ y $G_{2}$ tales que $\left(\left|G: G_{1}\right|,\left|G: G_{2}\right|\right)=1$, entonces $G$ es resoluble.

La existencia, conjugación y dominancia de los $\pi$-subgrupos de Hall está garantizada para grupos resolubles y también para la clase de grupos $\pi$-separables, para un conjunto arbitrario de primos $\pi$. En el siguiente resultado, Wielandt demuestra que la nilpotencia de los subgrupos de Hall de un grupo permite obtener propiedades similares en grupos finitos no necesariamente resolubles.

Teorema 2.12 (Wielandt). Sea $G$ un grupo finito con algún $\pi$-subgrupo $H$ de Hall, nilpotente. Entonces cada $\pi$-subgrupo de $G$ está contenido en un conjugado de $H$. En particular todos los $\pi$-subgrupos Hall de $G$ son conjugados.

Demostración. Véase 9.1.10 de [59].

También enunciamos dos resultados fundamentales de Burnside: la resolubilidad de los $\{p, q\}$-grupos y la no simplicidad de los grupos que poseen una clase de cardinal potencia de primo. 
Teorema 2.13 (Burnside). Si $|G|=p^{a} q^{b}$ con $p$ y $q$ dos primos distintos, entonces el grupo $G$ es resoluble.

Demostración. Véase 15.3 de [38].

Teorema 2.14 (Burnside). Si el grupo finito $G$ tiene una clase de conjugación con $p^{m}$ elementos $\left(p^{m}>1\right)$, para algún primo $p$, entonces $G$ no es simple.

Demostración. Véase 15.2 de [38].

En 1990, Kazarin extiende el Teorema 2.14 de Burnside, probando que el subgrupo generado por una clase de conjugación de cardinal potencia de primo es resoluble. En su demostración, Kazarin utiliza Teoría Modular de Representaciones.

Teorema 2.15 (Kazarin). Sea $G$ un grupo finito que tiene una clase de conjugación $x^{G}$ de tamaño potencia de un primo $p>1$, entonces $\left\langle x^{G}\right\rangle$ es subgrupo normal resoluble de $G$.

Demostración. Véase 15.7 de [38]. 


\section{Capítulo 3}

\section{Grupos con dos tamaños de clases de elementos $p$-regulares}

\subsection{Introducción}

Este capítulo está dedicado al estudio de la p-estructura del grupo a partir de la información obtenida sobre sus tamaños de clase de conjugación de sus elementos $p$-regulares, es decir, elementos cuyo orden no es divisible por el primo $p$. Esta es un área compleja y poco investigada, incluso bajo la hipótesis de $p$-resolubilidad del grupo, ya que si existe $p$-complemento, los tamaños de clases de los elementos en el $p$-complemento no dividen necesariamente a los tamaños de clases de dichos elementos en el grupo. Sin embargo, hemos podido comprobar que los resultados que se obtienen en este caso pueden aplicarse al estudio de la estructura del grupo a partir de sus tamaños de clase ordinarios.

En 1953, N. Itô estudia ([43]) la estructura de los grupos con dos tamaños de clases y obtiene que, salvo factores centrales, el grupo puede considerarse un $p$-grupo, para un cierto primo $p$.

Teorema 3.1 (Itô, 1953). Si G es un grupo finito con tamaños de clases de conjugación 1 y $m$, entonces $G=P \times A$, con $P$ un p-subgrupo de Sylow de $G$, para algún primo $p, y$ An subgrupo abeliano de $G$. Además, $m$ es potencia del primo $p$.

Posteriormente, en el año 2003, A. Beltrán y M.J. Felipe obtienen una generalización de este resultado, bajo la hipótesis de $p$-resolubilidad del grupo, para tamaños de clase de $p^{\prime}$-elementos, siendo $p$ un número primo, demostrando que en tales circunstancias el grupo tiene $p$-complemento abeliano ó factoriza como producto de un $\{p, q\}$-grupo por un grupo abeliano, con $q$ un primo distinto de $p$. 
Como hemos comentado en la introducción, en la demostración del citado resultado, Beltrán y Felipe se encuentran con una situación nueva, más compleja, en la que los centralizadores de los elementos $p$-regulares no centrales son todos conjugados, donde los autores hacen uso de la condición de $p$-resolubilidad del grupo. Obsérvese que para clases ordinarias esta situación no puede darse, mientras que, al trabajar con elementos $p$-regulares, es posible encontrar ejemplos de grupos en los que todos los centralizadores de los elementos $p$-regulares no centrales, para algún primo $p$, son conjugados, como veremos a continuación. Obsérvese que en este caso $G$ tiene exactamente dos tamaños de clase de conjugación de $p^{\prime}$-elementos.

Consideremos el producto semidirecto del grupo cuaternio $Q_{8}=\left\langle a, b: a^{4}=\right.$ $\left.1, a^{b}=a^{3}, a^{2}=b^{2}=(a b)^{2}\right\rangle$ y el grupo cíclico de orden $3, C_{3}=\langle\alpha\rangle$, dado por la acción de la siguiente permutación cíclica de orden 3 :

$$
\begin{gathered}
a^{\alpha}=b \\
b^{\alpha}=a b \\
(a b)^{\alpha}=a
\end{gathered}
$$

Claramente $G=Q_{8}\langle\alpha\rangle$ es grupo de orden 24 y tiene elementos de orden 2, 4, 3 y 6 . Si se considera $p=3$, los elementos $p$-regulares no centrales de $G$ son exactamente los elementos de $Q_{8} \backslash \mathbf{Z}\left(Q_{8}\right)=\left\{a, b, a b, a^{3}, b^{3},(a b)^{3}\right\}$. Es fácil ver que

$$
\begin{gathered}
\mathbf{C}_{G}(a)=\langle a\rangle=\mathbf{C}_{G}\left(a^{3}\right) \\
\mathbf{C}_{G}(b)=\langle b\rangle=\mathbf{C}_{G}\left(b^{3}\right) \\
\mathbf{C}_{G}(a b)=\langle a b\rangle=\mathbf{C}_{G}\left((a b)^{3}\right)
\end{gathered}
$$

por lo que sólo existen tres centralizadores distintos y todos ellos son de orden 4 . Por tanto, en este caso $m=6$ y $c s_{p^{\prime}}(G)=\{1,6\}$. Además, de la acción y de las propiedades de la conjugación, se deriva que $\mathbf{C}_{G}(a)=\mathbf{C}_{G}(a b)^{\alpha}=\mathbf{C}_{G}(b)^{\alpha^{2}}$.

En el año 1974, A.R. Camina prueba en ([22]) la resolubilidad de los grupos con dos tamaños de clases de conjugación de elementos $p$-regulares, para los que el orden del grupo cociente $G / \mathbf{Z}(G)$ es divisible al menos por dos primos distintos de $p$. En su trabajo Camina utiliza la clasificación de D. Gorenstein y J.H. Walter ([33]) sobre los grupos cuyos 2-subgrupos de Sylow son diédricos. En este capítulo se desarrolla una demostración alternativa, más simple, del resultado de Camina que permite eliminar la condición de $p$-resolubilidad del grupo en la hipótesis del teorema principal de [7], obteniéndose así una demostración conjunta más sencilla, que no hace uso de la Clasificación de Grupos Simples ó de resultados profundos de la Teoría de Grupos Finitos. 


\subsection{Resultados preliminares}

En el desarrollo del resultado principal del capítulo utilizaremos algunos lemas previos sobre tamaños de clases de $p^{\prime}$-elementos que presentamos a continuación. Empezamos con una caracterización de los grupos cuyos tamaños de clases de conjugación de $p^{\prime}$-elementos no centrales son potencias del primo $p$.

Lema 3.2 Sea $G$ un grupo finito. Entonces todos los tamaños de clases de conjugación en $G_{p^{\prime}}$ son potencias de $p$ si y sólo si $G$ tiene p-complemento abeliano, para un primo $p$.

Demostración. Mostraremos que en ambas direcciones de la demostración, el grupo $G$ de la hipótesis es resoluble. Supongamos primero que todos los tamaños de clases de conjugación de los $p^{\prime}$-elementos de $G$ son potencias de $p$ y apliquemos inducción sobre $|G|$. Por el Teorema 2.14 se tiene que $G$ es no simple y existe $N \triangleleft G$, con $N \neq 1$. Si $x \in N_{p^{\prime}}$, aplicando el Lema 2.4(a) obtenemos que $\left|x^{N}\right|$ divide a $\left|x^{G}\right|$ y por tanto $\left|x^{N}\right|$ es potencia de $p$. Por la hipótesis de inducción, $N$ es resoluble.

Sea $\bar{G}=G / N$ y sea $\bar{x} \in \bar{G}_{p^{\prime}}$. Aplicando ahora el Lema 2.4(b), se tiene que $\left|\bar{x}^{\bar{G}}\right|$ divide a $\left|x^{G}\right|$, y por tanto, $\left|\bar{x}^{G}\right|$ es potencia de $p$. De nuevo, por la hipótesis de inducción se tiene que $\bar{G}$ es resoluble. Como $N$ y $G / N$ son resolubles obtenemos que $G$ es resoluble.

Veamos ahora que si $G$ tiene $p$-complemento abeliano, entonces también es resoluble. Como $G$ tiene $p$-complemento abeliano, se puede escribir como producto de dos subgrupos nilpotentes, y por el Teorema 2.11 el grupo $G$ es resoluble también.

Demostraremos a continuación la doble implicación. Supongamos primero que $\left|x^{G}\right|$ es potencia de $p$ para todo $x \in G_{p^{\prime}}$. Trabajaremos por inducción sobre $|G|$ para mostrar que $G$ tiene $p$-complemento abeliano. Como $G$ es resoluble, en particular, es $p$-resoluble y por tanto $\mathbf{O}_{p}(G) \neq 1$ ó bien $\mathbf{O}_{p^{\prime}}(G) \neq 1$.

Supongamos que $\mathbf{O}_{p}(G) \neq 1$. Entonces $|\bar{G}|=\left|G / \mathbf{O}_{p}(G)\right|<|G|$ y por inducción obtenemos que $\bar{G}$ tiene $p$-complemento abeliano $\bar{H}$. Como $\bar{H}$ es $p$-resoluble, entonces $H$ puede escribirse como $H=H_{1} \mathbf{O}_{p}(G)$, siendo $H_{1}$ un $p$-complemento de $H$. Entonces

$$
\bar{H}=H / \mathbf{O}_{p}(G)=H_{1} \mathbf{O}_{p}(G) / \mathbf{O}_{p}(G) \cong H_{1} / H_{1} \cap \mathbf{O}_{p}(G)=H_{1}
$$

y obtenemos que $H_{1}$ es subgrupo abeliano de $G$ de orden un $p^{\prime}$-número. Como 


$$
|\bar{G}: \bar{H}|=\frac{|\bar{G}|}{|\bar{H}|}=\frac{\left|G / \mathbf{O}_{p}(G)\right|}{\left|H / \mathbf{O}_{p}(G)\right|}=\frac{|G|}{|H|}=|G: H|
$$

es potencia de $p$ también, se tiene que $\left|G: H_{1}\right|=|G: H|\left|H: H_{1}\right|$ es potencia de $p$ y $H_{1}$ es $p$-complemento abeliano de $G$. Podemos suponer, por tanto, que $\mathbf{O}_{p}(G)=1$ y $\mathbf{O}_{p^{\prime}}(G) \neq 1$. Consideremos el primer factor $N_{1}$ de la serie derivada de $G$

$$
1 \triangleleft N_{1} \triangleleft N_{2} \triangleleft \ldots \triangleleft N_{r}=G,
$$

donde $N_{i} \triangleleft G$, y $N_{i} / N_{i-1}$ es un factor abeliano. Entonces $N_{1}$ es subgrupo abeliano y por tanto nilpotente, por lo que $N_{1} \subseteq \mathbf{F}(G) \neq 1$. Sea $x$ un elemento no central de $G_{p^{\prime}}$. Como $G$ y $\mathbf{C}_{G}(x)$ son resolubles, y además $\left|G: \mathbf{C}_{G}(x)\right|$ es potencia del primo $p$, existe al menos un $p$-complemento $H$ de $G$ en $\mathbf{C}_{G}(x)$ con $x \in H \subseteq \mathbf{C}_{G}(x)$. Por otro lado, como $\mathbf{O}_{p}(G)=1$ se tiene que $\mathbf{F}(G)$ es un $p^{\prime}$-subgrupo normal de $G$ y $x \in \mathbf{C}_{G}(H) \subseteq \mathbf{C}_{G}(\mathbf{F}(G)) \subseteq \mathbf{F}(G) \subseteq \mathbf{O}_{p^{\prime}}(G)$.

Por tanto, todo elemento $p$-regular de $G$ está en $\mathbf{O}_{p^{\prime}}(G)$ y $H=\mathbf{O}_{p^{\prime}}(G)$ es $p$ complemento normal de $G$. Además, si $x \in H$, entonces $x \in \mathbf{C}_{G}(H) \subseteq H$. Es decir, $H=\mathbf{C}_{G}(H)$ y se tiene que $H$ es $p$-complemento abeliano.

Supongamos ahora que $G$ tiene $p$-complemento abeliano $H$. Entonces, por el Teorema 2.12, todo $p^{\prime}$-grupo está contenido en algún conjugado de $H$, que por hipótesis es abeliano. Sea $x \in G_{p^{\prime}}$. Entonces $\langle x\rangle \subseteq H^{g}$, para algún $g \in G$, por lo que $H^{g} \leq \mathbf{C}_{G}(x) \leq G$. Como $H^{g}$ es $p$-complemento de $G$ y por tanto de $\mathbf{C}_{G}(x)$, se tiene que $\left|x^{G}\right|$ es potencia de $p$ y se sigue que $\left|x^{G}\right|$ es potencia de $p$ para todo $x \in G_{p^{\prime}}$.

Recordemos que si $p$ es un primo fijo, el conjunto $G_{p^{\prime}}=\{g \in G: p$ no divide a $o(g)\}$ representa el conjunto de elementos $p$-regulares de $G$. El siguiente lema determina la estructura de los grupos cuyos tamaños de clases $p$-regulares no son divisibles por $p$, generalizando así el Lema 2.6 al caso $p$-regular.

Lema 3.3 Sea $G$ un grupo finito y $p$ un primo que no divide los tamaños de clase de conjugación de los elementos p-regulares de $G$. Entonces $G=P \times H$ donde $P$ es un p-subgrupo de Sylow de $G$ y $H$ es un p-complemento de $G$.

Demostración. Sea $P$ un $p$-subgrupo de Sylow de $G$ y $g \in G$. Consideremos la descomposición de $g$ en su $p$-parte y en su $p^{\prime}$-parte, $g=g_{p} g_{p^{\prime}}$. Como $\left|g_{p^{\prime}}{ }^{G}\right|$ es $p^{\prime}$-número, existe un $p$-subgrupo de Sylow de $G$, digamos $P^{t}$, tal que $P^{t} \subseteq \mathbf{C}_{G}\left(g_{p^{\prime}}\right)$. 
Al ser $g_{p}$ un $p$-elemento de $\mathbf{C}_{G}\left(g_{p^{\prime}}\right)$, se tiene que $g_{p}{ }^{c} \in P^{t}$, para algún $c \in \mathbf{C}_{G}\left(g_{p^{\prime}}\right)$, y por tanto $g^{c}=g_{p}{ }^{c} g_{p^{\prime}} \in P^{t} \mathbf{C}_{G}\left(P^{t}\right)$, de donde

$$
G=\bigcup_{t \in G} P^{t} \mathbf{C}_{G}\left(P^{t}\right)=\bigcup_{t \in G}\left(P \mathbf{C}_{G}(P)\right)^{t} .
$$

Concluimos que $G=P \mathbf{C}_{G}(P)$. Como $P$ y $\mathbf{C}_{G}(P)$ conmutan, se tiene que $P \unlhd G$ y, por tanto $G$ tiene una serie, $1 \unlhd P \unlhd G$, cuyos factores son, alternadamente, un $p$-grupo y un $p^{\prime}$-grupo. Por tanto $G$ es $p$-resoluble. En particular, se tiene que el $\mathbf{C}_{G}(P)$ es $p$-resoluble y tiene $p$-complemento $H$ que también es $p$-complemento de $G$. Por tanto, $G=P H$ donde $P$ y $H$ conmutan y $P \cap H=1$. Es decir, $G=P \times H$, con $H$ un $p$-complemento de $G$.

El siguiente resultado establece la forma de los centralizadores de los elementos $p$-regulares de un grupo $G$, que verifica que el centralizador de un $p^{\prime}$-elemento no puede contener al centralizador de otro $p^{\prime}$-elemento, a menos que los centralizadores coincidan ó uno de los $p^{\prime}$-elementos sea central.

Lema 3.4 Sea $G$ un grupo finito, $x \in G_{p^{\prime}}$ y $\mathbf{C}_{G}(x)<G$. Supongamos que para todo par de elementos $a, b \in G_{p^{\prime}}$ se verifica que si $\mathbf{C}_{G}(a) \leq \mathbf{C}_{G}(b)$ entonces $\mathbf{C}_{G}(a)=$ $\mathbf{C}_{G}(b)$ ób $\in \mathbf{Z}(G)$. Entonces $\boldsymbol{C}_{G}(x)=P \times L$, con $P$ un p-subgrupo de Sylow de $\boldsymbol{C}_{G}(x)$ y $L \leq \boldsymbol{Z}\left(\boldsymbol{C}_{G}(x)\right)$, ó $\boldsymbol{C}_{G}(x)=P Q \times A$, con $P$ un p-subgrupo de Sylow de $\boldsymbol{C}_{G}(x), Q$ un $q$-subgrupo de Sylow de $\boldsymbol{C}_{G}(x)$, para un cierto primo $q \neq p, y A \leq \boldsymbol{Z}(G)$.

Demostración. Por el Lema 2.1, el elemento $x$ se puede descomponer en producto de elementos de orden potencia de primos distintos de $p, x=x_{1} x_{2} \ldots x_{s}$, y las componentes conmutan dos a dos. Como $x \notin \mathbf{Z}(G)$, existe alguna componente $x_{i} \notin \mathbf{Z}(G)$. Además, $\mathbf{C}_{G}(x) \leq \mathbf{C}_{G}\left(x_{i}\right)$, y por hipótesis se tiene que $\mathbf{C}_{G}(x)=\mathbf{C}_{G}\left(x_{i}\right)$. Podemos por tanto asumir que $x$ es $q$-elemento, para un cierto primo $q \neq p$.

Si $\left|\mathbf{C}_{G}(x)\right|=p^{a} q^{b}$ el resultado es trivial. Supongamos que existe un primo $r$ distinto de $p$ y $q$ que divide a $\left|\mathbf{C}_{G}(x)\right|$ y sea $R$ un $r$-subgrupo de Sylow de $\mathbf{C}_{G}(x)$.

Si $y$ es un elemento de $R$, al ser $x$ e $y$ de orden coprimo y conmutar, se tiene que $\mathbf{C}_{G}(x y)=\mathbf{C}_{G}(x) \cap \mathbf{C}_{G}(y)$. Luego $\mathbf{C}_{G}(x y) \leq \mathbf{C}_{G}(x)$, y además $x y \in G_{p^{\prime}}$. Como $x$ es no central en $G$, se tiene que $\mathbf{C}_{G}(x)=\mathbf{C}_{G}(x y) \subseteq \mathbf{C}_{G}(y)$. Por tanto $y \in \mathbf{Z}\left(\mathbf{C}_{G}(x)\right)$ y se sigue que $R \leq \mathbf{Z}\left(\mathbf{C}_{G}(x)\right)$. Es decir $\mathbf{C}_{G}(x)=P Q \times A$, para algún $P \in \operatorname{Syl}_{p}\left(\mathbf{C}_{G}(x)\right)$, $Q \in \operatorname{Syl}_{q}\left(\mathbf{C}_{G}(x)\right)$ y $A \leq \mathbf{Z}\left(\mathbf{C}_{G}(x)\right)$.

Si $A \subseteq \mathbf{Z}(G)$ la prueba termina. Supongamos por tanto que existe un elemento no central $u \in A$. Como $u$ es un $\{p, q\}^{\prime}$-elemento, $u$ y $x$ son de orden coprimo y 
conmutan, por lo que $\mathbf{C}_{G}(u x)=\mathbf{C}_{G}(u) \cap \mathbf{C}_{G}(x)$. Luego $\mathbf{C}_{G}(u x) \leq \mathbf{C}_{G}(x)$ y de nuevo se tiene por hipótesis que $\mathbf{C}_{G}(u x)=\mathbf{C}_{G}(x) \subseteq \mathbf{C}_{G}(u)$. De manera análoga se obtiene que $\mathbf{C}_{G}(u x)=\mathbf{C}_{G}(x)=\mathbf{C}_{G}(u)$.

Tomemos ahora $z \in Q$. Entonces $z$ y $u$ conmutan y son de orden coprimo, por lo que $\mathbf{C}_{G}(u z)=\mathbf{C}_{G}(u) \cap \mathbf{C}_{G}(z)$. Es decir, $\mathbf{C}_{G}(u z) \leq \mathbf{C}_{G}(u)=\mathbf{C}_{G}(x)$ y por hipótesis $\mathbf{C}_{G}(u z)=\mathbf{C}_{G}(x) \leq \mathbf{C}_{G}(z)$, de donde $z \in \mathbf{Z}\left(\mathbf{C}_{G}(x)\right)$. Por tanto $Q \leq \mathbf{Z}\left(\mathbf{C}_{G}(x)\right)$. Podemos escribir $L=Q \times A$, de forma que $\mathbf{C}_{G}(x)=P \times L$ con $L \leq \mathbf{Z}\left(\mathbf{C}_{G}(x)\right)$.

Vamos a trabajar con subgrupos de un grupo de orden potencia de dos primos. Con este fin, demostraremos que los grupos de orden divisible por al menos dos primos distintos, $r$ y $s$, y cuyos subgrupos de Sylow son todos cíclicos, poseen subgrupos de orden rs. La demostración se apoya en el hecho de que tales subgrupos son metacíclicos.

Lema 3.5 (Hölder, Burnside, Zassenhaus). Sea $G$ un grupo finito con todos sus subgrupos de Sylow cíclicos, entonces $G$ es resoluble. Además, $G / G^{\prime}$ y $G^{\prime}$ son ambos cíclicos (de forma que $G$ es metacíclico), $G$ escinde sobre $G^{\prime}$ y $G^{\prime}$ es subgrupo de Hall de $G$.

Demostración. Véase 10.26 de [58].

Lema 3.6 Sea $G$ un grupo finito cuyos subgrupos de Sylow son todos cíclicos. Si $r$ y s son dos primos distintos que dividen a $|G|$, entonces existe un subgrupo $U$ de $G$ tal que $|U|=r s$.

Demostración. Sean $r$ y $s$ dos primos distintos que dividen a $|G|$. Veamos que $G$ tiene subgrupos de orden $r s$. Por el lema 3.5 se tiene que $G$ es metacíclico y $G^{\prime}$ es subgrupo normal cíclico de $G$ tal que $G / G^{\prime}$ es cíclico. Si $r$ y $s$ dividen a $\left|G^{\prime}\right|$, entonces $G^{\prime}$ contiene elementos de orden $r s$. Si $r$ y $s$ dividen a $\left|G / G^{\prime}\right|$ entonces $G / G^{\prime}$ contiene tales elementos, y por tanto $G$ también. Por último, si $r$ divide a $\left|G^{\prime}\right|$ y $s$ no, entonces existe un subgrupo normal $A$ de $G$ contenido en $G^{\prime}$ de orden $r$ y tomando un elemento $g \in G$ de orden $s$, se tiene que $A\langle g\rangle$ es subgrupo de $G$ de orden $r s$.

El siguiente resultado establece condiciones para que un grupo $K$ de orden divisible por un cierto primo $p$, que actúa como grupo de automorfismos de un $p$-grupo abeliano $P$, tenga $p^{\prime}$-radical trivial. 
Lema 3.7 Sea $P$ un p-grupo abeliano para un cierto primo $p$. Sea $K$ un grupo de automorfismos de $P$ tal que $|K|$ es divisible por $p$. Supongamos que $\mathbf{C}_{P}(x)=\mathbf{C}_{P}(y)$ para todo $x, y \in K \backslash\{1\}$. Entonces $\mathbf{O}_{p^{\prime}}(K)=1$.

Demostración. Sea $H=\mathbf{O}_{p^{\prime}}(K)$. Entonces $H$ actúa como grupo de automorfismos sobre $P$, que es grupo abeliano. Aplicando el Lema de Fitting obtenemos que $P=[P, H] \times \mathbf{C}_{P}(H)$. La hipótesis sobre los centralizadores puede escribirse equivalentemente en la forma $\mathbf{C}_{P}(x)=\mathbf{C}_{P}(L)$, para todo $x \in K$, no trivial, y $L \leq K$, subgrupo no trivial. Si $H \neq 1$, tenemos que $\mathbf{C}_{P}(x)=\mathbf{C}_{P}(H)$, para todo $x \in K$ no trivial. En particular, si $x$ es un $p$-elemento no trivial de $K$ entonces $x$ actúa sin puntos fijos sobre $[P, H]$, que es $p$-grupo, y esto sólo puede darse si $[P, H]=1$, es decir, si $H=\mathbf{O}_{p^{\prime}}(K)=1$.

A continuación enunciamos un resultado clásico de R. Brauer y M. Suzuki que recoge algunas propiedades de los grupos que poseen un 2-subgrupo de Sylow cuaternio generalizado.

Teorema 3.8 Sea $Q$ un 2-subgrupo de Sylow de $G$ y supongamos que $Q$ es un grupo cuaternio generalizado con única involución $j$. Entonces $\mathbf{O}_{2^{\prime}}(G)\langle j\rangle \triangleleft G$. En particular, $G$ no es simple.

Demostración. Véase 45.1 de [38].

\subsection{Resultado Principal}

En el desarrollo del resultado principal del capítulo seguiremos el esquema de la demostración dada por Beltrán y Felipe en [7]. Trabajaremos por pasos y asumiremos que existen al menos tres primos distintos que dividen el orden del grupo cociente $G / \mathbf{Z}(G)$, separando el desarrollo en dos partes. Por una parte se estudia el caso en que los centralizadores de los elementos $p$-regulares no centrales no son todos conjugados, y por otra, el caso en que sí lo son. Como ya hemos comentado en la introducción esta situación es factible cuando se trabaja con $p^{\prime}$-elementos. Nuestro resultado principal es el siguiente.

Teorema 3.9 Sea $G$ un grupo finito con tamaños de clase de conjugación de elementos p-regulares 1 y $m$. Entonces $m=p^{a} q^{b}$, con $q$ un primo distinto de $p$ y $a, b \geq 0$. Si $b=0$ entonces $G$ tiene $p$-complemento abeliano. Si $b \neq 0$, entonces $G=P Q \times A$, con $P \in \operatorname{Syl}_{p}(G), Q \in \operatorname{Syl}_{q}(G)$ y $A \leq \mathbf{Z}(G)$. Si $a=0$ entonces $G=P \times Q \times A$, con $A \leq \mathbf{Z}(G)$. En particular, se obtiene que $G$ es resoluble. 
Demostración. Por el Lema 3.2, si $m=p^{a}$ entonces $G$ tiene $p$-complemento abeliano y este caso está demostrado. Por lo tanto asumiremos que $b \neq 0$ y veremos que $m=p^{a} q^{b}$, con $a \geq 0$. Si $a=0$, la afirmación en la tesis se seguirá como consecuencia trivial del Lema 3.3.

Procederemos por pasos y por inducción sobre el orden de $G$. En los pasos 1 y 2 se estudia la forma que tienen los centralizadores de los elementos $p$-regulares no centrales de $G$ y cómo se relacionan entre sí.

Paso 1. Podemos asumir que $\mathbf{C}_{G}(x)=P_{x} \times L_{x}$, con $P_{x}$ un $p$-subgrupo de Sylow de $\mathbf{C}_{G}(x)$ y $L_{x} \leq \mathbf{Z}\left(\mathbf{C}_{G}(x)\right)$, para todo $x \in G_{p^{\prime}}$ no central.

Por el Lema 3.4 sabemos que para cualquier $x \in G_{p^{\prime}} \backslash \mathbf{Z}(G)$ se tiene que $\mathbf{C}_{G}(x)=P_{x} \times L_{x}$, con $P_{x}$ un $p$-subgrupo de Sylow de $\mathbf{C}_{G}(x)$ y $L_{x} \leq \mathbf{Z}\left(\mathbf{C}_{G}(x)\right)$, o $\mathbf{C}_{G}(x)=P_{x} Q_{x} \times A$, con $P_{x}$ y $Q_{x}$ un $p$-subgrupo y un $q$-subgrupo de Sylow de $\mathbf{C}_{G}(x)$, respectivamente, y $A \leq \mathbf{Z}(G)$.

Supongamos que existe un elemento $x \in G_{p^{\prime}} \backslash \mathbf{Z}(G)$ tal que $\mathbf{C}_{G}(x)=P_{x} Q_{x} \times A$. Veremos que en este caso el teorema se verifica. Procederemos por reducción al absurdo. Supongamos que $G \neq P Q \times A$. Entonces podemos asumir que existe un $r$-elemento $z$ no central en $G$, para algún primo $r$ distinto de $p$ y de $q$, tal que:

$$
A<\langle A, z\rangle \leq \mathbf{C}_{G}(z)
$$

Comparando el orden de los subgrupos se tiene que:

$$
|A|<|\langle A, z\rangle| \leq\left|\mathbf{C}_{G}(z)\right|_{\{p, q\}^{\prime}}=\left|\mathbf{C}_{G}(x)\right|_{\{p, q\}^{\prime}}=|A|
$$

obteniéndose una contradicción, por lo que $G=P Q \times A$ para algún $P \in \operatorname{Syl}_{p}(G)$ y $Q \in \operatorname{Syl}_{q}(G)$, y se tiene el resultado.

Paso 2. Sean $x$ e $y$ dos elementos $p$-regulares no centrales de $G$. Si $\mathbf{C}_{G}(x) \neq$ $\mathbf{C}_{G}(y)$, entonces $\left(\mathbf{C}_{G}(x) \cap \mathbf{C}_{G}(y)\right)_{p^{\prime}}=\mathbf{Z}(G)_{p^{\prime}}$.

Supongamos que existe un elemento no central $a \in\left(\mathbf{C}_{G}(x) \cap \mathbf{C}_{G}(y)\right)_{p^{\prime}}$. Como $a$ es un elemento $p$-regular de ambos centralizadores, por el Paso 1, tiene que estar en el $p$-complemento de cada uno de ellos. Es decir, $a \in L_{x} \leq \mathbf{Z}\left(\mathbf{C}_{G}(x)\right)$, y de manera análoga, $a \in L_{y} \leq \mathbf{Z}\left(\mathbf{C}_{G}(y)\right)$. Por tanto $\mathbf{C}_{G}(x) \subseteq \mathbf{C}_{G}(a)$ y $\mathbf{C}_{G}(y) \subseteq \mathbf{C}_{G}(a)$, y por órdenes se tiene que $\mathbf{C}_{G}(x)=\mathbf{C}_{G}(y)=\mathbf{C}_{G}(a)$, llegándose a una contradicción.

Para el resto de la demostración distinguiremos dos casos, según que los centralizadores de los elementos $p$-regulares no centrales de $G$ sean todos conjugados o no. 
CASO 1. Supongamos que los centralizadores de los elementos no centrales de $G_{p^{\prime}}$ no son todos conjugados en $G$.

Denotaremos por $\bar{G}=G / \mathbf{Z}(G)_{p^{\prime}}$ y utilizaremos barras para trabajar en el grupo cociente. En los pasos 3, 4 y 5 se demuestra que cada elemento $p$-regular del grupo cociente es de orden un primo distinto de $p$.

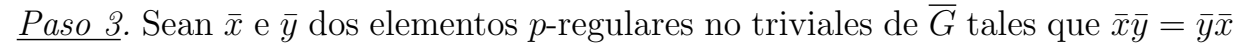
y $\mathbf{C}_{G}(x) \neq \mathbf{C}_{G}(y)$. Entonces $o(\bar{x})=o(\bar{y})$ es primo.

Nótese que $x, y$ y $x y$ son también elementos $p$-regulares de $G$. Supongamos que $o(\bar{x})<o(\bar{y})$, entonces $(\bar{x} \bar{y})^{o(\bar{x})}=(\bar{y})^{o(\bar{x})} \neq 1$. Por lo tanto, $1 \neq(\bar{x} \bar{y})^{o(\bar{x})}=(\overline{x y})^{o(\bar{x})} \in$ $\overline{\mathbf{C}_{G}(x y)} \cap \overline{\mathbf{C}_{G}(y)}$, y por el Paso 2 se tiene que $\mathbf{C}_{G}(y)=\mathbf{C}_{G}(x y)$. Luego, $x \in \mathbf{C}_{G}(y)$, y aplicando de nuevo el Paso 2 obtenemos que $\mathbf{C}_{G}(x)=\mathbf{C}_{G}(y)$, llegándose a una contradicción. Concluimos que $o(\bar{x})=o(\bar{y})$.

Veamos ahora que $o(\bar{x})=o(\bar{y})$ es primo. Si $s$ es un primo divisor de $o(\bar{x}) \mathrm{y}$ $\bar{x}^{s} \neq 1$, entonces $\mathbf{C}_{G}(x) \subseteq \mathbf{C}_{G}\left(x^{s}\right)<G$ y como los centralizadores de los elementos $p$-regulares no centrales son del mismo orden, obtenemos que $\mathbf{C}_{G}(x)=\mathbf{C}_{G}\left(x^{s}\right)$. Por otro lado, $\bar{x}^{s} \bar{y}=\bar{y} \bar{x}^{s}$ y como $\mathbf{C}_{G}\left(x^{s}\right)=\mathbf{C}_{G}(x) \neq \mathbf{C}_{G}(y)$ se obtiene que $o\left(\bar{x}^{s}\right)=$ $o(\bar{y})=o(\bar{x})$ y llegamos a una contradicción.

Paso 4. Sea $g$ un elemento no central de $G_{p^{\prime}}$ y consideremos la clase de conjugación $\bar{g} \bar{G}$. Entonces existe algún elemento no central $x \in G_{p^{\prime}}$ tal que $\bar{g}^{\bar{G}} \cap \overline{\mathbf{C}_{G}(x)}=\emptyset$.

Supongamos que $\bar{g}^{\bar{G}} \cap \overline{\mathbf{C}_{G}(x)} \neq \emptyset$. Entonces, para cada elemento no central $x \in G_{p^{\prime}}$, se tiene que $\overline{\mathbf{C}_{G}(x)}$ contiene algún conjugado $\bar{g}^{\bar{n}}$ de $\bar{g}$, para algún $\bar{n} \in \bar{G}$. Por tanto $\overline{g^{n}} \in \overline{\mathbf{C}_{G}(x)}$, y como $g$ es $p^{\prime}$-elemento de $G, g^{n} \in \mathbf{C}_{G}(x)_{p^{\prime}}=L_{x}$. Por el Paso 1 , se sigue que $\left.g^{n} \in \mathbf{Z}\left(\mathbf{C}_{G}(x)\right)\right)$ y por tanto $\mathbf{C}_{G}(x) \leq \mathbf{C}_{G}\left(g^{n}\right)$, y por órdenes, la igualdad se verifica. Es decir $\mathbf{C}_{G}(x)=\mathbf{C}_{G}\left(g^{n}\right)=\mathbf{C}_{G}(g)^{n}$, por lo que dos centralizadores cualesquiera son conjugados en $G$, obteniéndose una contradicción.

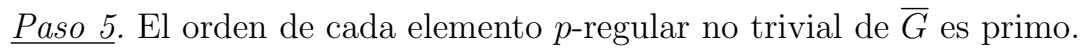

Supongamos que existe algún elemento $p$-regular $\bar{g} \neq 1$ tal que $o(\bar{g})$ no es primo. Obsérvese que $g^{n}$ y $g$ son elementos $p$-regulares de $G$ también. Por el Paso 4 existe algún elemento no central $x \in G_{p^{\prime}}$ tal que $\bar{g}^{\bar{G}} \cap \overline{\mathbf{C}_{G}(x)}=\emptyset$. Supongamos que existe algún elemento no trivial $\bar{y} \in \overline{\mathbf{C}_{G}(x)_{p^{\prime}}}$ que centraliza a algún conjugado $\bar{g}^{\bar{n}}$ de $\bar{g}$. 
Como $x$ es no central, por el Paso 2 se tiene que $\mathbf{C}_{G}(x)=\mathbf{C}_{G}(y)$. Entonces, o bien se verifica que

$$
\overline{\mathbf{C}_{G}(x)}=\overline{\mathbf{C}_{G}(y)}=\overline{\mathbf{C}_{G}\left(g^{n}\right)},
$$

lo que nos lleva a que $\overline{g^{n}} \in \bar{g}^{\bar{G}} \cap \overline{\mathbf{C}_{G}(x)}$, contradiciendo el Paso 4, o bien se verifica que

$$
\overline{\mathbf{C}_{G}(x)}=\overline{\mathbf{C}_{G}(y)} \neq \overline{\mathbf{C}_{G}\left(g^{n}\right)},
$$

lo que, por el Paso 3, implica que $\bar{x}, \overline{g^{n}}$ y por tanto $\bar{g}$ son de orden primo, y obtenemos de nuevo una contradicción. Concluimos que no existen elementos no triviales en $\overline{\mathbf{C}_{G}(x)_{p^{\prime}}}$ que centralicen a conjugados de $\bar{g}$.

Denotaremos por $\overline{C_{p^{\prime}}}$ a $\overline{\mathbf{C}_{G}(x)_{p^{\prime}}}$. Entonces $\overline{C_{p^{\prime}}}$ actúa por conjugación como grupo de permutaciones sobre $\bar{g}$, de manera que el estabilizador en cada punto $\bar{g}^{\bar{h}} \in \bar{g}^{\bar{G}}$ viene dado por

$$
\operatorname{Stab}_{\overline{C_{p^{\prime}}}}\left(\bar{g}^{\bar{h}}\right)=\left\{\bar{w} \in \overline{C_{p^{\prime}}}:\left(\bar{g}^{\bar{h}}\right)^{\bar{w}}=\bar{g}^{\bar{h}}\right\}=1
$$

obteniéndose que la acción es semirregular, por lo que las órbitas son todas del mismo orden y $\left|\overline{C_{p^{\prime}}}\right|$ divide a $\left|\bar{g}^{\bar{G}}\right|$.

Veamos ahora que la acción de $\overline{\mathbf{C}_{G}(g)_{p^{\prime}}}$ sobre el conjunto $X=\bar{g}^{\bar{G}} \backslash \bar{g}^{\bar{G}} \cap \overline{\mathbf{C}_{G}(g)}$ es también semirregular. Si $\bar{z} \in \overline{\mathbf{C}_{G}(g)_{p^{\prime}}}$ fija algún conjugado $\bar{g}^{\bar{n}} \in \bar{g}^{\bar{G}}$, como $\bar{g}^{\bar{n}}$ no es de orden primo, por el Paso 3 se sigue que $\mathbf{C}_{G}(z)=\mathbf{C}_{G}\left(g^{n}\right)$. Y como $z$ y $g$ conmutan, obtenemos que $g \in \mathbf{C}_{G}\left(g^{n}\right)$, es decir $\bar{g}^{\bar{n}} \in \bar{g}^{\bar{G}} \cap \overline{\mathbf{C}_{G}(g)}$, por lo que el estabilizador de cada elemento de $X$ es 1 . Por tanto $\left|\overline{\mathbf{C}_{G}(g)_{p^{\prime}}}\right|=\left|\overline{C_{p^{\prime}}}\right|$ divide a $|X|$, de donde $\left|\overline{\mathbf{C}_{G}(g)_{p^{\prime}}}\right|$ divide a $\left|\bar{g}^{\bar{G}} \cap \overline{\mathbf{C}_{G}(g)}\right|$, y, como $\bar{g}^{\bar{G}} \subseteq \bar{G}_{p^{\prime}}$, se obtiene la contradicción

$$
\left|\overline{\mathbf{C}_{G}(g)_{p^{\prime}}}\right| \leq\left|\bar{g} \bar{G} \cap \overline{\mathbf{C}_{G}(g)_{p^{\prime}}}\right|<\left|\overline{\mathbf{C}_{G}(g)_{p^{\prime}}}\right| .
$$

En el siguiente paso, utilizamos los tres últimos resultados para comprobar que el grupo cociente es $\{p, q\}$-grupo, y así, obtener la conclusión en el Caso 1.

$\underline{\text { Paso 6. }}$. Conclusión en el Caso 1.

Sea $q$ un primo distinto de $p$ divisor de $\left|G / \mathbf{Z}(G)_{p^{\prime}}\right|$ y sea $x$ un $q$-elemento no central de $G$. Como todos los elementos $p$-regulares no triviales de $G / \mathbf{Z}(G)_{p^{\prime}}$ son de orden primo, se tiene que $\overline{\mathbf{C}_{G}(x)}$ es un $\{p, q\}$-grupo. Como todos los elementos $p$-regulares no centrales de $G$ tienen centralizadores del mismo orden, las imágenes de estos centralizadores en $G / \mathbf{Z}(G)_{p^{\prime}}$ son $\{p, q\}$-grupos y, en particular, no puede 
haber primos divisores de $\left|G / \mathbf{Z}(G)_{p^{\prime}}\right|$ distintos de $p$ y $q$. Por tanto $G$ puede escribirse en la forma $G=P Q \times A$, con $P \in \operatorname{Syl}_{p}(G), Q \in \operatorname{Syl}_{q}(G)$ y $A \leq \mathbf{Z}(G)$.

En este punto iniciamos la demostración del resultado para el Caso 2.

CASO 2. Supongamos que para cualquier par de elementos no centrales $x, y \in G_{p^{\prime}}$, $\mathbf{C}_{G}(x)$ y $\mathbf{C}_{G}(y)$ son conjugados en $G$.

En los pasos 7, 8 y 9 se demuestra que si el grupo es resoluble el teorema se verifica.

Paso 7. Podemos asumir que $\mathbf{O}^{p}(G)=G$.

Aplicaremos inducción para demostrar que si $\mathbf{O}^{p}(G)<G$ el teorema se cumple. Sea $x$ un elemento $p$-regular no central de $\mathbf{O}^{p}(G)$, y por tanto no central en $G$. Por el Paso 1 se tiene que $\mathbf{C}_{G}(x)=P_{x} \times L_{x}$, con $P_{x}$ un $p$-subgrupo de Sylow de $\mathbf{C}_{G}(x)$ y $L_{x} \leq \mathbf{Z}\left(\mathbf{C}_{G}(x)\right)$. Al ser $p^{\prime}$-grupo, $L_{x} \subseteq \mathbf{O}^{p}(G)$, y aplicando la igualdad de Dedekind a $\mathbf{O}^{p}(G) \cap \mathbf{C}_{G}(x)$ se tiene que

$$
\mathbf{O}^{p}(G) \cap \mathbf{C}_{G}(x)=L_{x}\left(P_{x} \cap \mathbf{O}^{p}(G)\right),
$$

de donde

$$
\frac{|G|}{\left|\mathbf{O}^{p}(G)\right|} \frac{\left|\mathbf{O}^{p}(G)\right|}{\left|\mathbf{O}^{p}(G) \cap \mathbf{C}_{G}(x)\right|}=\frac{|G|}{\left|\mathbf{C}_{G}(x)\right|} \frac{\left|\mathbf{C}_{G}(x)\right|}{\left|\mathbf{O}^{p}(G) \cap \mathbf{C}_{G}(x)\right|}=m \frac{\left|P_{x}\right|}{\left|P_{x} \cap \mathbf{O}^{p}(G)\right|},
$$

por lo que

$$
\frac{\left|\mathbf{O}^{p}(G)\right|}{\left|\mathbf{O}^{p}(G) \cap \mathbf{C}_{G}(x)\right|}=\frac{m\left|P_{x}\right|}{\left|G: \mathbf{O}^{p}(G)\right|\left|P_{x} \cap \mathbf{O}^{p}(G)\right|} .
$$

Es decir,

$$
\left|x^{\mathbf{O}^{p}(G)}\right|=\frac{m\left|P_{x}\right|}{l\left|P_{x} \cap \mathbf{O}^{p}(G)\right|},
$$

donde $l=\left|G: \mathbf{O}^{p}(G)\right|$ es un entero positivo. Sea $y$ otro elemento $p$-regular no central de $\mathbf{O}^{p}(G)$, con centralizador $\mathbf{C}_{G}(y)=P_{y} \times L_{y}$, siendo $P_{y} \in \operatorname{Syl}_{p}\left(\mathbf{C}_{G}(y)\right)$ y $L_{y} \in \mathbf{Z}\left(\mathbf{C}_{G}(y)\right)$. Como los centralizadores de los $p^{\prime}$-elementos no centrales de $G$ tienen la misma forma y son del mismo orden, se sigue que $\left|P_{y}\right|=\left|P_{x}\right|$ y $\left|L_{y}\right|=\left|L_{x}\right|$, de manera que 


$$
\left|P_{y} \cap \mathbf{O}^{p}(G)\right|=\left|\left(P_{x}\right)^{h} \cap\left(\mathbf{O}^{p}(G)\right)^{h}\right|=\left|\left(P_{x} \cap \mathbf{O}^{p}(G)\right)^{h}\right|=\left|P_{x} \cap \mathbf{O}^{p}(G)\right| .
$$

Por tanto,

$$
\left|y^{\mathbf{O}^{p}(G)}\right|=\frac{m\left|P_{y}\right|}{l\left|P_{y} \cap \mathbf{O}^{p}(G)\right|}=\left|x^{\mathbf{O}^{p}(G)}\right|=k
$$

y se tiene que $\mathbf{O}^{p}(G)$ tiene dos tamaños de clases de conjugación de $p^{\prime}$-elementos. Al ser $\left|\mathbf{O}^{p}(G)\right|<|G|$, aplicando la hipótesis inductiva obtenemos que $\mathbf{O}^{p}(G)$ tiene $p$-complemento abeliano o bien $\mathbf{O}^{p}(G)=P_{0} Q \times A_{0}$, con $P_{0}$ un $p$-subgrupo de Sylow de $\mathbf{O}^{p}(G), Q$ un $q$-subgrupo de Sylow de $\mathbf{O}^{p}(G)$, para algún primo $q \neq p$, y $A_{0} \leq \mathbf{Z}\left(\mathbf{O}^{p}(G)\right)$.

Si $H$ es un $p$-complemento abeliano de $\mathbf{O}^{p}(G)$, entonces los índices $\left|G: \mathbf{O}^{p}(G)\right|$ y $\left|\mathbf{O}^{p}(G): H\right|$ son potencias de $p$, por lo que $|G: H|$ también es potencia de $p$, y por tanto $H$ es $p$-complemento de $G$.

Si $\mathbf{O}^{p}(G)=P_{0} Q \times A_{0}$ con $Q \leq \mathbf{O}^{p}(G) \leq G$, entonces $\left|G: \mathbf{O}^{p}(G)\right|$ es potencia de $p$, por lo que $|G|_{q}=\left|\mathbf{O}^{p}(G)\right|_{q}=|Q|$ y se concluye que $Q \in \operatorname{Syl}_{q}(G)$. Veamos que $A_{0} \leq \mathbf{Z}(G)_{p^{\prime}}$. Si existen dos elementos no centrales $x$ e $y$ de $G_{p^{\prime}}$ tales que $\mathbf{C}_{G}(x) \neq \mathbf{C}_{G}(y)$, entonces por el Paso 2 se tiene que $\left(\mathbf{C}_{G}(x) \cap \mathbf{C}_{G}(y)\right)_{p^{\prime}}=$ $\mathbf{Z}(G)_{p^{\prime}}$. Como $x, y \in \mathbf{O}^{p}(G)$ y $A_{0}$ es $p^{\prime}$-grupo central de $\mathbf{O}^{p}(G)$ se sigue que $A_{0} \subseteq\left(\mathbf{C}_{G}(x) \cap \mathbf{C}_{G}(y)\right)_{p^{\prime}}=\mathbf{Z}(G)_{p^{\prime}} \leq \mathbf{Z}(G)$. Por tanto, el grupo $T=Q \times A_{0}$ es p-complemento de $\mathbf{O}^{p}(G)$ y también de $G$ por lo que podemos escribir $G=P Q \times A_{0}$, con $P \in \operatorname{Syl}_{p}(G), Q \in \operatorname{Syl}_{q}(G)$ y $A_{0} \leq \mathbf{Z}(G)$.

Si no existen dos elementos no centrales en $G_{p^{\prime}}$ tales que $\mathbf{C}_{G}(x) \neq \mathbf{C}_{G}(y)$, entonces los centralizadores de los elementos $p$-regulares no centrales son iguales, por lo que $|G|_{q}=\left|\mathbf{C}_{G}(x)\right|_{q}$, para cada $x \in G_{p^{\prime}}$, no central, y cada $q \neq p$. Se sigue que $\left|x^{G}\right|$ es potencia de $p$ y por el Lema 3.2 se concluye que $G$ tiene $p$-complemento abeliano.

Paso 8. $\mathbf{C}_{G}(x)<\mathbf{N}_{G}\left(\mathbf{C}_{G}(x)\right)$ para todo $x \in G_{p^{\prime}}$ no central.

Supongamos que $\mathbf{N}_{G}\left(\mathbf{C}_{G}(x)\right)=\mathbf{C}_{G}(x)$, para algún $x \in G_{p^{\prime}}$ no central. Como $m$ no es potencia de $p$, existe algún primo $q \neq p$ que divide a $m$. Por el Paso 1 , $\mathbf{C}_{G}(x)=P_{x} \times L_{x}$, con $P_{x} \in \operatorname{Syl}_{p}\left(\mathbf{C}_{G}(x)\right)$ y $L_{x}$ abeliano. Veamos que $L_{x}$ contiene $q$-elementos no centrales en $G$, es decir que $q$ también divide a $\left|L_{x} / \mathbf{Z}(G)_{p^{\prime}}\right|$. Supongamos que $q$ no divide a $\left|L_{x} / \mathbf{Z}(G)_{p^{\prime}}\right|$ y sea $g$ un $q$-elemento no central de $G$. Como $\mathbf{C}_{G}(g)=P_{g} \times L_{g}$ y los centralizadores de elementos $p$-regulares no centrales son del mismo orden, se tiene que $\left|L_{g}\right|=\left|L_{x}\right|$, y por tanto, $q$ no divide a $\left|L_{g} / \mathbf{Z}(G)_{p^{\prime}}\right|$, por lo que $L_{g} / \mathbf{Z}(G)_{p^{\prime}}$ no tiene $q$-elementos no triviales. Pero $g \mathbf{Z}(G)_{p^{\prime}}$ es un $q$-elemento no 
trivial del grupo cociente, obteniéndose una contradicción. Concluimos que $q$ divide a $\left|L_{x} / \mathbf{Z}(G)_{p^{\prime}}\right|$.

Sea $L_{i}$ el $q$-subgrupo de Sylow de $\mathbf{C}_{G}(x)$, no central en $G$, y sea $Q_{i}$ un $q$-subgrupo de Sylow de $G$, tal que $L_{i} \subseteq Q_{i}$. Entonces $L_{i}<\mathbf{N}_{Q_{i}}\left(L_{i}\right) \subseteq \mathbf{N}_{G}\left(L_{i}\right)$. Sea $y$ un elemento de $\mathbf{N}_{Q_{i}}\left(L_{i}\right) \backslash L_{i} \subseteq \mathbf{N}_{G}\left(L_{i}\right) \backslash L_{i}$ y sea $\mathbf{C}_{G}\left(x^{y}\right)=\mathbf{C}_{G}(x)^{y}=\left(P_{x}\right)^{y} \times\left(L_{x}\right)^{y}$. Si $\left(L_{x}\right)^{y} \neq L_{x}$ entonces $\mathbf{C}_{G}\left(x^{y}\right) \neq \mathbf{C}_{G}(x)$, y por el Paso 2 se tiene $\mathbf{C}_{G}(x)_{p^{\prime}} \cap \mathbf{C}_{G}\left(x^{y}\right)_{p^{\prime}}=$ $L_{x} \cap\left(L_{x}\right)^{y}=\mathbf{Z}(G)_{p^{\prime}}$. Como $L_{i}^{y} \leq L_{x}^{y}$ entonces $L_{i} \leq\left(L_{x}\right)^{y} \cap L_{x}=\mathbf{Z}(G)_{p^{\prime}}, \mathrm{y}$ se obtiene una contradicción. Concluimos que $\left(L_{x}\right)^{y}=L_{x}$. Por otro lado, $L_{x}<$ $\mathbf{Z}\left(\mathbf{C}_{G}(x)\right)$, de donde

$$
P_{x} \subseteq \mathbf{C}_{G}\left(L_{x}\right)=\mathbf{C}_{G}\left(\left(L_{x}\right)^{y}\right) \subseteq \mathbf{C}_{G}\left(x^{y}\right) .
$$

Por tanto $P_{x}=P_{x}^{y}$ y se tiene que $\mathbf{C}_{G}(x)=\mathbf{C}_{G}(x)^{y}$, por lo que $y \in \mathbf{N}_{G}\left(\mathbf{C}_{G}(x)\right)=$ $\mathbf{C}_{G}(x)$. Como $y$ es $q$-elemento de $\mathbf{C}_{G}(x)$, que es nilpotente, se tiene que $y \in L_{i}, \mathrm{y}$ obtenemos de nuevo una contradicción. Concluimos que $\mathbf{C}_{G}(x)<\mathbf{N}_{G}\left(\mathbf{C}_{G}(x)\right)$.

Paso 9. Si el grupo es resoluble el teorema se verifica.

Por el Paso 7, podemos suponer $\mathbf{O}^{p^{\prime}}(G)<G$. Sea $x \in G_{p^{\prime}}$ un elemento fijo no central y sea $g \in G$. Podemos escribir $g=g_{p} g_{p^{\prime}}$, donde $g_{p}$ y $g_{p^{\prime}}$ son la $p$-parte y la $p^{\prime}$-parte de $g$, respectivamente. Si $g_{p^{\prime}} \in \mathbf{Z}(G)$, entonces $g \in \mathbf{Z}(G)_{p^{\prime}} \mathbf{O}^{p^{\prime}}(G)$. Si $g_{p^{\prime}} \notin \mathbf{Z}(G)$, como los centralizadores de los elementos $p$-regulares no centrales son todos conjugados, existe un $n \in G$ tal que $g \in \mathbf{C}_{G}(g) \subseteq \mathbf{C}_{G}\left(g_{p^{\prime}}\right)=\mathbf{C}_{G}(x)^{n}$. Por tanto,

$$
G=\bigcup_{n \in G} \mathbf{C}_{G}(x)^{n} \bigcup_{n \in G}\left(\mathbf{O}^{p^{\prime}}(G)\right) \mathbf{Z}(G)_{p^{\prime}} .
$$

Como el número de conjugados distintos de $\mathbf{C}_{G}(x)$ es $\left|G: \mathbf{N}_{G}\left(\mathbf{C}_{G}(x)\right)\right|$, se tiene que

$$
\left.|G| \leq\left|G: \mathbf{N}_{G}\left(\mathbf{C}_{G}(x)\right)\right|\left(\left|\mathbf{C}_{G}(x)\right|-1\right)+\mid \mathbf{O}^{p^{\prime}}(G)\right) \mathbf{Z}(G)_{p^{\prime}} \mid
$$

y dividiendo por $|G|$ se tiene que

$$
1 \leq \frac{\left|\mathbf{C}_{G}(x)\right|-1}{\left|\mathbf{N}_{G}\left(\mathbf{C}_{G}(x)\right)\right|}+\frac{\left|\mathbf{O}^{p^{\prime}}(G) \mathbf{Z}(G)_{p^{\prime}}\right|}{|G|} .
$$

Por el Paso 8, tenemos que $\mathbf{C}_{G}(x)<N_{G}\left(\mathbf{C}_{G}(x)\right)$, por lo que existen al menos 2 coclases de $\mathbf{C}_{G}(x)$ en $\mathbf{N}_{G}\left(\mathbf{C}_{G}(x)\right)$. Aplicando el teorema de la transitividad de índices se tiene que 


$$
\left|\mathbf{N}_{G}\left(\mathbf{C}_{G}(x)\right)\right|=\left|\mathbf{N}_{G}\left(\mathbf{C}_{G}(x)\right): \mathbf{C}_{G}(x)\right|\left|\mathbf{C}_{G}(x)\right| \geq 2\left|\mathbf{C}_{G}(x)\right| .
$$

Sea $\left|\mathbf{N}_{G}\left(\mathbf{C}_{G}(x)\right)\right|=n_{1}$. Si $\mathbf{O}^{p^{\prime}}(G) \mathbf{Z}(G)_{p^{\prime}}<G$, entonces se obtiene que $|G| \geq$ $2\left|\mathbf{O}^{p^{\prime}}(G) \mathbf{Z}(G)_{p^{\prime}}\right|$ y sustituyendo en la ecuación 3.1 se sigue que

$$
1 \leq \frac{\left|\mathbf{C}_{G}(x)\right|}{2\left|\mathbf{C}_{G}(x)\right|}-\frac{1}{n_{1}}+\frac{\left|\mathbf{O}^{p^{\prime}}(G) \mathbf{Z}(G)_{p^{\prime}}\right|}{2\left|\mathbf{O}^{p^{\prime}}(G) \mathbf{Z}(G)_{p^{\prime}}\right|}=1-\frac{1}{n_{1}}
$$

y llegamos a una contradicción. Concluimos que $\mathbf{O}^{p^{\prime}}(G) \mathbf{Z}(G)_{p^{\prime}}=G$.

Supongamos que $\mathbf{O}^{p^{\prime}}(G)$ no es $p$-grupo, ya que en tal caso $G$ tiene $p$-complemento abeliano, y sea $y$ un elemento $p$-regular de $\mathbf{O}^{p^{\prime}}(G)$, no central en $G$. Entonces $\left|y^{G}\right|=$ $\left|y^{\mathbf{O}^{p^{\prime}}(G)}\right|=m$ y podemos aplicar inducción y concluir que $\mathbf{O}^{p^{\prime}}(G)=P Q_{0} \times S_{0}$, con $P \in \operatorname{Syl}_{p}(G), Q_{0} \in \operatorname{Syl}_{q}\left(\mathbf{O}^{p^{\prime}}(G)\right)$, para algún primo $q \neq p$, y $S_{0} \leq \mathbf{Z}\left(\mathbf{O}^{p^{\prime}}(G)\right) \leq \mathbf{Z}(G)$. Por tanto, $G=\mathbf{O}^{p^{\prime}}(G) \mathbf{Z}(G)_{p^{\prime}}=P Q \times A$, con $A \leq \mathbf{Z}(G)$ y $Q \in \operatorname{Syl}_{q}(G)$.

En los pasos 10 y 11 demostraremos que el índice $\left|\mathbf{N}_{G}\left(\mathbf{C}_{G}(x)\right) / \mathbf{C}_{G}(x)\right|$ es un primo $q$ distinto de $p$ para cada $x \in G_{p^{\prime}}$.

Paso 10. Si $x$ es un $p^{\prime}$-elemento no central de $G$, cada subgrupo de Sylow de $\mathbf{N}_{G}\left(\mathbf{C}_{G}(x)\right) / \mathbf{C}_{G}(x)$ es cíclico o cuaternio generalizado. Es más, si $q \neq p$ es un primo divisor del orden de este grupo cociente entonces el $q$-subgrupo de Sylow tiene orden $q$.

Sea $x \in G_{p^{\prime}} \backslash \mathbf{Z}(G)$ y sea $W=\mathbf{N}_{G}\left(\mathbf{C}_{G}(x)\right) / \mathbf{C}_{G}(x)$. Usaremos barras para trabajar en el grupo cociente. Sea $Q$ un $q$-subgrupo de Sylow de $W$ para algún primo $q$ que divide a $|W|$ (posiblemente $q=p$ ). Por los supuestos iniciales del teorema existe algún primo $r$, distinto de $p$ y $q$, que divide a $|G / \mathbf{Z}(G)|$. Es decir, existe un elemento $g_{r}$ de orden potencia de $r$ en $G \backslash \mathbf{Z}(G)$, y $r$ divide a $\left|\mathbf{C}_{G}\left(g_{r}\right)\right|=\left|\mathbf{C}_{G}(x)\right|$.

Por tanto existe un $r$-subgrupo de Sylow $R_{x}$ de $\mathbf{C}_{G}(x)$ no trivial. Como $\mathbf{C}_{G}(x)$ es nilpotente, $R_{x}$ es $W$-invariante, y así $Q$ actúa sobre $R_{x}$. Sea $\psi$ el automorfismo representación de $Q$ correspondiente a dicha acción. Entonces

$$
\begin{aligned}
\operatorname{Ker}(\psi) & =\left\{\bar{g} \in Q: w^{g}=w, \text { para todo } w \in R_{x}, g \in \mathbf{N}_{G}\left(\mathbf{C}_{G}(x)\right)\right\} \\
& =\left\{\bar{g} \in Q: g \in \mathbf{C}_{\mathbf{N}_{G}\left(\mathbf{C}_{G}(x)\right)}\left(R_{x}\right)\right\} \subseteq\left\{\bar{g} \in Q: g \in \mathbf{C}_{G}(z), z \in R_{x}\right\} \\
& =\left\{\bar{g} \in Q: g \in \mathbf{C}_{G}(x)\right\}=\overline{\mathbf{C}_{G}(x)}=1,
\end{aligned}
$$

por lo que $\psi$ es inyectiva y $Q$ actúa como grupo de automorfismos sobre $R_{x}$. 
Obsérvese que si $\bar{g} \in Q, \bar{g} \neq 1$, entonces $\mathbf{C}_{R_{x}}(\bar{g})=R_{x} \cap \mathbf{Z}(G)$. Basta comprobar que si $z \in \mathbf{C}_{R_{x}}(\bar{g})$, entonces $z \in \mathbf{Z}(G)$. Sea $g \in \mathbf{N}_{G}\left(\mathbf{C}_{G}(x)\right)$ tal que $\bar{g}$ es un elemento no trivial de $Q$. Supongamos que existe $z \in \mathbf{C}_{R_{x}}(\bar{g}) \backslash \mathbf{Z}(G)$. Entonces $z \in R_{x} \leq L_{x} \leq \mathbf{Z}\left(\mathbf{C}_{G}(x)\right)$, y por el Paso 1 se tiene que $\mathbf{C}_{G}(z)=\mathbf{C}_{G}(x)$. Como $z \in \mathbf{C}_{R_{x}}(\bar{g})$, entonces $g \in \mathbf{C}_{G}(z)=\mathbf{C}_{G}(x)$, por lo que $\bar{g}=1$ y llegamos a una contradicción.

$\mathrm{Al}$ ser $\left(|Q|,\left|R_{x}\right|\right)=1$, la acción es coprima, y como $R_{x}$ es abeliano podemos aplicar el Teorema 2.8 y concluir que $R_{x}=\left[R_{x}, Q\right] \times \mathbf{C}_{R_{x}}(Q)$. Además $\left[R_{x}, Q\right] \neq 1$, ya que de lo contrario $R_{x} \subseteq \mathbf{C}_{R_{x}}(\bar{g}) \subseteq \mathbf{Z}(G)$ y $R_{x}$ sería $r$-subgrupo de Sylow de $\mathbf{C}_{G}\left(g_{r}\right)$, por lo que $g_{r} \in R_{x} \subseteq \mathbf{Z}(G)$, lo que es falso.

Veamos ahora que la acción de $Q$ sobre $\left[R_{x}, Q\right]$ no tiene puntos fijos. Basta comprobar que si $t$ es un elemento no trivial de $\left[R_{x}, Q\right]$ y $\bar{g}$ es un elemento no trivial de $Q$, entonces $t^{\bar{g}} \neq t$. Supongamos que $t^{\bar{g}}=t$, entonces $t \in \mathbf{C}_{\left[R_{x}, Q\right]}(\bar{g}) \leq \mathbf{C}_{R_{x}}(\bar{g})=$ $R_{x} \cap \mathbf{Z}(G)$. Luego $t \in \mathbf{Z}(G)$ y se tiene que $t^{g}=t$, para todo $g \in G$. Se sigue que $t^{\bar{g}}=t$, para todo $\bar{g} \in Q$, y por tanto, $t \in \mathbf{C}_{R_{x}}(Q)$. Pero $\left[R_{x}, Q\right] \cap \mathbf{C}_{R_{x}}(Q)=1$, por lo que $t=1$, y obtenemos una contradicción. Aplicando el Teorema 2.9 se concluye que $Q$ es cíclico o cuaternio generalizado.

Supongamos ahora que $q \neq p$ y consideremos el $q$-subgrupo de Sylow $Q_{x}$ de $\mathbf{C}_{G}(x)$. Sea $\bar{Q}_{x}=Q_{x} / \mathbf{Z}(G)_{q}$. Como $Q_{x}$ es característico en $\mathbf{C}_{G}(x)$, se tiene que $\bar{Q}_{x}$ es $W$-invariante, y podemos considerar la acción por conjugación del grupo $Q$ sobre $\bar{Q}_{x}$. Sea $M=\overline{Q_{x}} Q$ el producto semidirecto definido por dicha acción. Por construcción $M$ es $q$-grupo y $\bar{Q}_{x} \unlhd M$, por lo que $\bar{Q}_{x} \cap \mathbf{Z}(M)$ es un $q$-subgrupo no trivial de $M$ y tiene elementos de orden $q$. Sea $\bar{t}$ uno de tales elementos y consideremos el subgrupo $T=\langle t\rangle \mathbf{Z}(G)_{q} \leq Q_{x} \leq \mathbf{C}_{G}(x)$. Entonces $Q$ actúa sobre $T$ por conjugación. Veamos que $\mathbf{C}_{Q}(T)=1$. Como $t$ es un $q$-elemento de $Q_{x}$ no central en $G$, se tiene que $\mathbf{C}_{G}(t)=$ $\mathbf{C}_{G}(x)$, por lo que, si $\bar{g} \in \mathbf{C}_{Q}(T)$ y $h \in T$, entonces $h^{\bar{g}}=h^{g}=h$ y obtenemos que $g \in \mathbf{C}_{G}(h)=\mathbf{C}_{G}(x)$, de donde $\bar{g} \in \overline{\mathbf{C}_{G}(x)}=1$, como se quería demostrar. Obsérvese que $[T, Q] \subseteq \mathbf{Z}(G)_{q}$, ya que, como $t \in \mathbf{Z}(M)$ y $\bar{g} \in Q \leq M$, entonces $t^{\bar{g}}=\overline{t^{g}}=\bar{t}$ y podemos escribir $t^{g}=t a$, con $a \in \mathbf{Z}(G)_{q}$, por lo que $t^{-1} t^{g} \in \mathbf{Z}(G)_{q}$. Por tanto, $[t, g]$ y $t^{q}$ son elementos de $\mathbf{Z}(G)_{q}$, y se tiene que $[t, g]^{q}=\left[t^{q}, g\right]=1=\left[t, g^{q}\right]$. Luego $\bar{g}^{q} \in \mathbf{C}_{Q}(T)=1$ y así $Q$ tiene exponente $q$. Como además es cíclico o cuaternio, $Q$ debe tener orden exactamente $q$.

Paso 11. Para todo $x \in G_{p^{\prime}}$, se tiene que $\left|\mathbf{N}_{G}\left(\mathbf{C}_{G}(x)\right) / \mathbf{C}_{G}(x)\right|=q$, para algún primo $q$ distinto de $p$.

Primero probaremos que $W=\mathbf{N}_{G}\left(\mathbf{C}_{G}(x)\right) / \mathbf{C}_{G}(x)$ es un $q$-grupo para algún primo $q$ (posiblemente $q=p$ ). Supongamos que $|W|$ es divisible por al menos dos 
primos distintos. Demostraremos que existe un subgrupo $U$ de $W$ tal que $|U|$ es producto de dos números primos.

Por el Paso 10, todo subgrupo de Sylow de $W$ es cíclico o cuaternio generalizado. Si todos los Sylows de $W$ son cíclicos entonces por el Lema 3.6 podemos concluir que existe tal subgrupo $U$. Si algún 2-subgrupo de Sylow de $W$ es cuaternio generalizado podemos aplicar el Teorema 3.8 para concluir que $\mathbf{O}_{2^{\prime}}(W)\langle\tau\rangle \unlhd W$, donde $\tau$ es la única involución de $W$. Como todos los $2^{\prime}$-subgrupos de Sylow de $W$ son cíclicos, los de $\mathbf{O}_{2^{\prime}}(W)$ también lo son. Por tanto, si $\left|\mathbf{O}_{2^{\prime}}(W)\right|$ es divisible, como mínimo, por dos primos distintos, aplicando el Lema 3.6 podemos afirmar que tal subgrupo $U$ existe. Si $\left|\mathbf{O}_{2^{\prime}}(W)\right|$ es divisible por un único primo, es decir $\mathbf{O}_{2^{\prime}}(W)$ es $r$-grupo cíclico para algún primo $r \neq 2$, entonces existe un elemento $\alpha \in \mathbf{O}_{2^{\prime}}(W)$ de orden $r$ y podemos construir el subgrupo $U=\langle\alpha\rangle\langle\tau\rangle$ de orden $2 r$. Concluimos que en todos los casos existe un subgrupo $U \leq W$ de orden $r s$, con $r$ y $s$ dos primos distintos.

Veamos ahora que esto nos lleva a una contradicción. Si ambos primos son distintos de $p$, el orden de $U$ es producto de dos primos y por tanto $U$ tiene $r$-complemento o $s$-complemento. Notar que, por los teoremas de Sylow, uno de los complementos es normal en $U$. Concretamente si $r<s$, el $s$-subgrupo de Sylow es normal, y si $s<r$ entonces, el $r$-subgrupo de Sylow es normal. Asumiremos sin pérdida de generalidad, que el $r$-complemento es normal. Si uno de los primos es $p$, entonces existe un primo $s$ distinto de $p$ y $r$ tal que $s$ divide a $|G / \mathbf{Z}(G)|$ y por tanto $G$ tiene un $s$-elemento $g$ no central y $s$ divide $\left|\mathbf{C}_{G}(g)\right|=\left|\mathbf{C}_{G}(x)\right|$.

Sea $S_{x}$ el $s$-subgrupo de Sylow de $\mathbf{C}_{G}(x)$. Entonces $S_{x}=\left[S_{x}, U\right] \times \mathbf{C}_{S_{x}}(U)$. Afirmamos que si $\bar{y} \in U$ es no trivial, entonces $\mathbf{C}_{S_{x}}(y) \subseteq \mathbf{Z}(G)$. En efecto, ya que en caso contrario existe algún elemento no central $z \in S_{x}$ que conmuta con $y$, y por el Paso 1, se tiene que $\mathbf{C}_{G}(x) \subseteq \mathbf{C}_{G}(z)$ y, como ambos centralizadores tienen el mismo orden, se sigue que $\mathbf{C}_{G}(x)=\mathbf{C}_{G}(z)$, resultando la contradicción $y \in \mathbf{C}_{G}(x)$. Entonces, si $\left[S_{x}, U\right]=1$ se tiene que $S_{x} \subseteq \mathbf{Z}(G)$, y por tanto, $S_{x}$ también sería $s$-subgrupo de Sylow de $\mathbf{C}_{G}(g)$, de donde $g \in S_{x} \subseteq \mathbf{Z}(G)$, lo que es falso. Por tanto $\left[S_{x}, U\right] \neq 1$ y de hecho $U$ actúa sin puntos fijos sobre este grupo dado que, si $\bar{y} \in U$ es no trivial,

$$
\mathbf{C}_{\left[S_{x}, U\right]}(y) \subseteq\left[S_{x}, U\right] \cap \mathbf{C}_{S_{x}}(y) \subseteq\left[S_{x}, U\right] \cap \mathbf{Z}(G) \subseteq\left[S_{x}, U\right] \cap \mathbf{C}_{S_{x}}(U)=1 .
$$

Si aplicamos ahora, por ejemplo, el Lema 16.12 de [38], obtenemos que $U$ es cíclico, y en particular $U$ tiene $r$-complemento normal no trivial. Es decir, en ambos casos se concluye que $U$ tiene $r$-complemento normal para algún primo $r$ distinto de $p$. 
Consideremos la acción de $U$ por conjugación, como grupo de automorfismos de $R_{x}$, siendo $R_{x}$ un $r$-subgrupo de Sylow abeliano de $\mathbf{C}_{G}(x)$. Obsérvese que si $\bar{u}$ y $\bar{v}$ son elementos no triviales de $U$, entonces

$$
\mathbf{C}_{R_{x}}(\bar{u})=\mathbf{C}_{R_{x}}(\bar{v})=R_{x} \cap \mathbf{Z}(G)=\mathbf{Z}(G)_{r}
$$

y aplicando el Lema 3.7 obtenemos que $\mathbf{O}_{r^{\prime}}(U)=1$, llegándose a una contradicción. Concluimos que $\left|\mathbf{N}_{G}\left(\mathbf{C}_{G}(x)\right) / \mathbf{C}_{G}(x)\right|$ es potencia de un primo.

Veamos ahora que este primo no es $p$. Si $p$ es el único primo que divide a $m$, aplicando el Lema 3.2 se concluye que $G$ tiene $p$-complemento abeliano. Sea $r$ un primo distinto de $p$ que divide a $m$. Entonces $r$ divide a $|G / \mathbf{Z}(G)|$, y por tanto $G$ tiene $r$-elementos no centrales y los centralizadores de los $p^{\prime}$-elementos no centrales también los tienen. Sea $R_{x}$ el $r$-subgrupo de Sylow de $\mathbf{C}_{G}(x)$. Nótese que $R_{x}$ no es $r$-subgrupo de Sylow de $G$, pues de lo contrario $r$ no divide a $m$. Si $t \in R_{x}$ es un elemento no central de $G$, entonces $\mathbf{C}_{G}(x)=\mathbf{C}_{G}(t)$. Si $w \in \mathbf{N}_{G}\left(R_{x}\right)$ entonces $\mathbf{C}_{G}\left(t^{w}\right)=\mathbf{C}_{G}(t)=\mathbf{C}_{G}(x)$, de forma que $\mathbf{C}_{G}(x)=\mathbf{C}_{G}(t)^{w}=\mathbf{C}_{G}(x)^{w}$ y $w \in$ $\mathbf{N}_{G}\left(\mathbf{C}_{G}(x)\right)$. Por consiguiente, $\mathbf{N}_{G}\left(R_{x}\right) \leq \mathbf{N}_{G}\left(\mathbf{C}_{G}(x)\right)$. Como $R_{x}$ no es subgrupo de Sylow de $G$, existe un $r$-subgrupo de Sylow $R$ de $G$ tal que $R_{x} \leq R$, de forma que $R_{x}<\mathbf{N}_{R}\left(R_{x}\right)$ y se tiene

$$
\mathbf{C}_{G}(x)_{r}=R_{x}<\mathbf{N}_{R}\left(R_{x}\right) \leq \mathbf{N}_{G}\left(R_{x}\right) \leq \mathbf{N}_{G}\left(\mathbf{C}_{G}(x)\right),
$$

lo que implica que $r$ divide a $|W|$. Por tanto, $W$ es un $q$-grupo para un cierto primo $q$ distinto de $p$ y por el Paso 10, el orden del $q$-grupo es exactamente $q$.

Como resultado del Paso 11 y del hecho de que todos los centralizadores de los elementos $p$-regulares no centrales de $G$ son conjugados, podemos asumir que

$$
\left|\mathbf{N}_{G}\left(\mathbf{C}_{G}(x)\right) / \mathbf{C}_{G}(x)\right|=q
$$

para un primo fijo $q$ distinto de $p$ y para todo $x \in G_{p^{\prime}}$ no central. Por lo tanto, los normalizadores de los centralizadores de los elementos $p$-regulares no centrales de $G$ son todos del mismo orden.

En los pasos 12 y 13 se demuestra que el $r$-radical de $G$ es trivial si $r=p$ y es central si $r \neq p$. Estos resultados se utilizan en el paso 14, donde se presenta la conclusión del caso 2 .

Paso 12. Podemos asumir que $\mathbf{O}_{p}(G)=1$ y que $\left|G: \mathbf{N}_{G}\left(\mathbf{C}_{G}(x)\right)\right|$ es potencia de $p$ para cada $x \in G_{p^{\prime}}$ no central. 
Sea $x \in G_{p^{\prime}}$ no central y sea $R$ un $r$-subgrupo de Sylow de $G$, para un primo $r$ distinto de $p$. Si $R$ es central en $G$ entonces $R \leq \mathbf{C}_{G}(x)<\mathbf{N}_{G}\left(\mathbf{C}_{G}(x)\right)$ y $r$ no divide a $\left|G: \mathbf{N}_{G}\left(\mathbf{C}_{G}(x)\right)\right|$. Si $R$ es subgrupo abeliano no central de $G$ entonces existe un elemento no central $y \in R$ tal que $R \leq \mathbf{C}_{G}(y) \leq G$. Como $\left|\mathbf{C}_{G}(y)\right|_{r}=\left|\mathbf{C}_{G}(x)\right|_{r}=|G|_{r}$, se tiene que $r$ no divide a $\left|G: \mathbf{C}_{G}(x)\right|$. Y por el Paso 8, el primo $r$ tampoco divide a $\mid G: \mathbf{N}_{G}\left(\mathbf{C}_{G}(x) \mid\right.$.

Si $R$ no es abeliano entonces $R \backslash \mathbf{Z}(R) \neq 1$, por lo que $R / \mathbf{Z}(R)$ es un $r$-grupo con centro no trivial. Sea $\bar{t}$ un elemento no trivial de $\mathbf{Z}(R / \mathbf{Z}(R))$ y sea $w \in R$. Veamos que $\mathbf{C}_{R}(t)=\mathbf{C}_{R}\left(t^{w}\right)=\mathbf{C}_{R}(t)^{w}$. Como $\bar{t} \in \mathbf{Z}(R / \mathbf{Z}(R))$ y $\bar{w} \in R / \mathbf{Z}(R)$, se sigue que $[\bar{t}, \bar{w}]=1$ y por tanto $[t, w] \in \mathbf{Z}(R)$. Entonces, si $g \in \mathbf{C}_{R}(t)$ entonces $t^{g}=t$, de manera que $\left(t^{w}\right)^{g}=t^{w}$ y concluimos que $g \in \mathbf{C}_{R}\left(t^{w}\right)$. Por otro lado, si $g \in \mathbf{C}_{R}\left(t^{w}\right)$ entonces $\left(t^{w}\right)^{g}=t^{g} t^{-1} t^{w}=t^{w}$, por lo que $t^{g}=t$ y $g \in \mathbf{C}_{R}(t)$. Por consiguiente, $\mathbf{C}_{R}(t)=\mathbf{C}_{R}\left(t^{w}\right)=\mathbf{C}_{R}(t)^{w}$, y concluimos que $\mathbf{C}_{R}(t) \unlhd R$.

Como los centralizadores de los elementos p-regulares no centrales son todos conjugados, existe $h \in G$ tal que $\mathbf{C}_{G}(t)=\mathbf{C}_{G}(x)^{h}=\mathbf{C}_{G}\left(x^{h}\right)$, con $x^{h} \in G_{p^{\prime}}$ no central.

Dado un elemento $g \in \mathbf{N}_{G}\left(\mathbf{C}_{R}(t)\right)$, se tiene que $t^{g} \in \mathbf{C}_{R}(t) \leq \mathbf{C}_{G}(t)=\mathbf{C}_{G}\left(x^{h}\right)$. Luego $t^{g}$ es $r$-elemento del $\mathbf{C}_{G}\left(x^{h}\right)$, y aplicando el Paso 1, se tiene que $t^{g} \in R_{x} \leq$ $\mathbf{Z}\left(\mathbf{C}_{G}\left(x^{h}\right)\right)$. Por tanto, $\mathbf{C}_{G}\left(x^{h}\right) \leq \mathbf{C}_{G}\left(t^{g}\right)$, y por órdenes coinciden. Es decir,

$$
\mathbf{C}_{G}(t)=\mathbf{C}_{G}\left(x^{h}\right)=\mathbf{C}_{G}\left(t^{g}\right)=\mathbf{C}_{G}(t)^{g}=\mathbf{C}_{G}\left(x^{h}\right)^{g},
$$

obteniéndose que $g \in \mathbf{N}_{G}\left(\mathbf{C}_{G}\left(x^{h}\right)\right)$. Concluimos que $R \leq \mathbf{N}_{G}\left(\mathbf{C}_{R}(t)\right) \leq \mathbf{N}_{G}\left(\mathbf{C}_{G}\left(x^{h}\right)\right)$ y $r$ no divide a $\left|G: \mathbf{N}_{G}\left(\mathbf{C}_{G}\left(x^{h}\right)\right)\right|$. Como los normalizadores de los elementos $p$ regulares no centrales de $G$ son del mismo orden, sus índices en $G$ coinciden, por lo que $r$ tampoco divide a $\left|G: \mathbf{N}_{G}\left(\mathbf{C}_{G}(x)\right)\right|$. Obtenemos así que en todos los casos $\left|G: \mathbf{N}_{G}\left(\mathbf{C}_{G}(x)\right)\right|$ es potencia de $p$.

Asumiremos ahora que $\mathbf{O}_{p}(G) \neq 1$, y aplicando inducción, veremos que en este caso el teorema se verifica. Utilizaremos barras para trabajar en el grupo cociente $\bar{G}=G / \mathbf{O}_{p}(G)$. Sea $x \in G_{p^{\prime}}$ y sea $\bar{y} \in \mathbf{C}_{\bar{G}}(\bar{x})$. Entonces $[x, y] \in \mathbf{O}_{p}(G)$, por lo que $x^{y}=x k$, con $x$ un elemento de $\mathbf{C}_{G}(x)$ y $k$ un elemento de $\mathbf{O}_{p}(G)$, de manera que $x^{y}$ es un $p^{\prime}$-elemento de $\mathbf{C}_{G}(x) \mathbf{O}_{p}(G)$.

Como $\mathbf{C}_{G}(x)=P_{x} \times L_{x}$, con $L_{x}$ un $p^{\prime}$-subgrupo del centralizador, y por tanto, $L_{x}$ es un $p$-complemento de $\mathbf{C}_{G}(x) \mathbf{O}_{p}(G)$, se tiene que

$$
x^{y} \in L_{x}{ }^{h}=L_{x}^{a b}=L_{x}^{b}
$$


donde $h=a b$ es un elemento de $\mathbf{C}_{G}(x) \mathbf{O}_{p}(G)$, con $a \in \mathbf{C}_{G}(x)$ y $b \in \mathbf{O}_{p}(G)$. Obtenemos que $x^{y b^{-1}} \in L_{x}$, y por el Paso 1 ,

$$
\mathbf{C}_{G}(x)=\mathbf{C}_{G}\left(x^{y b^{-1}}\right)=\mathbf{C}_{G}(x)^{y b^{-1}} .
$$

Por tanto, $y b^{-1} \in \mathbf{N}_{G}\left(\mathbf{C}_{G}(x)\right)$ y podemos poner $y=w b$, con $w \in \mathbf{N}_{G}\left(\mathbf{C}_{G}(x)\right)$. Como $b \in \mathbf{O}_{p}(G)$ se tiene que $\bar{y}=\bar{w}$, y por tanto $\bar{w} \in \mathbf{C}_{\bar{G}}(\bar{x})$. Es decir $\bar{w} \bar{x}=\bar{x} \bar{w} \mathrm{y}$ $[w, x] \in \mathbf{O}_{p}(G)$ es $p$-elemento de $G$.

Como $w \in \mathbf{N}_{G}\left(\mathbf{C}_{G}(x)\right)$, se tiene que $x^{w} \in L_{x} \leq \mathbf{C}_{G}(x)$ y $x^{-1} x^{w} \in L_{x}$, por ser producto de $p^{\prime}$-elementos que conmutan. Es decir, $[w, x]$ es $p^{\prime}$-elemento también. Por consiguiente, $[w, x]=1$ y se tiene que $w \in \mathbf{C}_{G}(x)$ y $\bar{w} \in \overline{\mathbf{C}_{G}(x)}$. Pero $\bar{w}=$ $\bar{y} \in \overline{\mathbf{C}_{G}(x)}$, por lo que $\mathbf{C}_{\bar{G}}(\bar{x}) \subseteq \overline{\mathbf{C}_{G}(x)}$ y se tiene la igualdad, $\mathbf{C}_{\bar{G}}(\bar{x})=\overline{\mathbf{C}_{G}(x)}$. Concluimos que $\left|\bar{G}: \mathbf{C}_{\bar{G}}(\bar{x})\right|=\left|\bar{G}: \overline{\mathbf{C}_{G}(x)}\right|=\left|G: \mathbf{C}_{G}(x) \mathbf{O}_{p}(G)\right|$ y, como los centralizadores son todos conjugados y $\mathbf{O}_{p}(G)$ es normal en $G$, este último índice es el mismo para todo $x \in G_{p^{\prime}}$. Concluimos que $\bar{G}$ tiene dos tamaños de clase de conjugación de elementos $p$-regulares. Aplicando inducción obtenemos que $\bar{G}$ tiene $p$-complemento abeliano, de manera que $G$ tiene $p$-complemento abeliano también, o $\bar{G}=\overline{P Q} \times \bar{A}$, con $\bar{P} \in \operatorname{Syl}_{p}(\bar{G}), \bar{Q} \in \operatorname{Syl}_{q}(\bar{G})$ y $\bar{A} \leq \mathbf{Z}(\bar{G})$. En el primer caso la demostración concluye. En el segundo, se obtiene que $G$ es resoluble, por serlo $\bar{G}$ y $\mathrm{O}_{p}(G)$ y, por el Paso 9, el resultado se tiene.

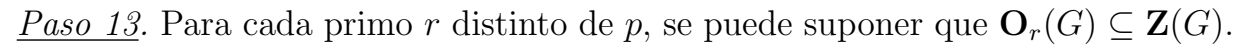

Sea $r$ un primo distinto de $p$ y supongamos que $K=\mathbf{O}_{r}(G)$ es subgrupo no central de $G$. Por el Paso 12 se tiene que $\left|G: N\left(\mathbf{C}_{G}(x)\right)\right|$ es potencia de $p$, y por tanto $K \subseteq \mathbf{N}_{G}\left(\mathbf{C}_{G}(x)\right)$, para todo $p^{\prime}$-elemento $x$ de $G$. Por hipótesis, existen al menos tres primos distintos que dividen a $|G / \mathbf{Z}(G)|$. Si $s$ es un primo distinto de $p$ y $r$ que divide a $|G / \mathbf{Z}(G)|$, entonces $s$ también divide a $\left|\mathbf{C}_{G}(x) / \mathbf{Z}(G)\right|$.

Sea $S_{x}$ el $s$-subgrupo de Sylow de $\mathbf{C}_{G}(x)$, no central en $G$. Nótese que si $y \in S_{x} \subseteq$ $\mathbf{C}_{G}(x)$ y $g \in K \leq \mathbf{N}_{G}\left(\mathbf{C}_{G}(x)\right)$, entonces $y^{g}$ es $s$-elemento de $\mathbf{C}_{G}(x)$ y por tanto $K$ normaliza $S_{x}$ y se tiene que $\left[S_{x}, K\right] \subseteq S_{x} \cap K=1$. Es decir, $K \subseteq \mathbf{C}_{G}\left(S_{x}\right)=\mathbf{C}_{G}(x)$, donde la última igualdad es consecuencia del Paso 1. Si $t \in K \backslash \mathbf{Z}(G)$ entonces $\mathbf{C}_{G}(t)=\mathbf{C}_{G}(x)$. Es más, si $w \in \mathbf{N}_{G}(K)$ entonces $t^{w}$ es $r$-elemento no central de $G$, por lo que $\mathbf{C}_{G}\left(t^{w}\right)=\mathbf{C}_{G}(x)=\mathbf{C}_{G}(t)=\mathbf{C}_{G}(t)^{w}=\mathbf{C}_{G}(x)^{w}$ y se tiene que $w \in$ $\mathbf{N}_{G}\left(\mathbf{C}_{G}(x)\right)$. Esto implica que $\mathbf{N}_{G}(K)=G \subseteq \mathbf{N}_{G}\left(\mathbf{C}_{G}(x)\right)$ y por tanto $\mathbf{C}_{G}(x) \unlhd G$, concluyéndose que los centralizadores de los elementos $p$-regulares no centrales de $G$ coinciden, por lo que todos los $p^{\prime}$-elementos conmutan y $G$ tiene $p$-complemento abeliano. 


\section{Paso 14. Conclusión.}

Por el Paso 11 se tiene que $\left|\mathbf{N}_{G}\left(\mathbf{C}_{G}(x)\right) / \mathbf{C}_{G}(x)\right|=q$, para un primo $q \neq p$ divisor de $|G|$ y, por tanto, $q$ divide a $m$. Obsérvese que si $\mathbf{Z}(G)_{q}=1$, entonces $G$ tiene un $q$-subgrupo de Sylow $Q$ no central en $G$, y si $g$ es un elemento no trivial de $\mathbf{Z}(Q)$, entonces $Q \leq \mathbf{C}_{G}(g)$ y $|Q|=\left|\mathbf{C}_{G}(g)\right|_{q}=|G|_{q}$, por lo que $q$ no divide a $m$ y llegamos a una contradicción. Por lo tanto, $\mathbf{Z}(G)_{q} \neq 1$ y podemos considerar el grupo cociente $\bar{G}=G / \mathbf{Z}(G)_{q}$. Probaremos que $\bar{G}$ tiene dos tamaños de clase de conjugación de elementos $p$-regulares.

Asumiremos que $\bar{G}$ no es abeliano, ya que de serlo, $G$ es resoluble y la demostración termina. Si $\bar{a} \in \bar{G}_{p^{\prime}} \backslash \mathbf{Z}(\bar{G})$, trivialmente se tiene que $\overline{\mathbf{C}_{G}(a)} \subseteq \mathbf{C}_{\bar{G}}(\bar{a})$. Veamos que $\overline{\mathbf{C}_{G}(a)}=\mathbf{C}_{\bar{G}}(\bar{a})$, por lo que $\left|\bar{G}: \mathbf{C}_{\bar{G}}(\bar{a})\right|=m$ y el grupo cociente $\bar{G}$ tiene dos tamaños de clases de $p^{\prime}$-elementos.

Supongamos que existe un elemento $p$-regular $\bar{a} \in \bar{G}$ tal que $\overline{\mathbf{C}_{G}(a)} \neq \mathbf{C}_{\bar{G}}(\bar{a})$. Si $\bar{w} \in \mathbf{C}_{\bar{G}}(\bar{a})$ entonces $w \in \mathbf{N}_{G}\left(\mathbf{C}_{G}(a)\right)$, por lo que $\mathbf{C}_{\bar{G}}(\bar{a}) \subseteq \overline{\mathbf{N}_{G}\left(\mathbf{C}_{G}(a)\right)}$. Aplicando el Paso 11 se tiene que $\left|\overline{\mathbf{N}_{G}\left(\mathbf{C}_{G}(a)\right)}: \overline{\mathbf{C}_{G}(a)}\right|=\left|\mathbf{N}_{G}\left(\mathbf{C}_{G}(a)\right): \mathbf{C}_{G}(a)\right|=q$. Como $\overline{\mathbf{C}_{G}(a)}<\mathbf{C}_{\bar{G}}(\bar{a}) \leq \overline{\mathbf{N}_{G}\left(\mathbf{C}_{G}(a)\right)}$, obtenemos que

$$
\left|\overline{\mathbf{N}_{G}\left(\mathbf{C}_{G}(a)\right)}: \overline{\mathbf{C}_{G}(a)}\right|=\left|\overline{\mathbf{N}_{G}\left(\mathbf{C}_{G}(a)\right)}: \mathbf{C}_{\bar{G}}(\bar{a})\right|\left|\mathbf{C}_{\bar{G}}(\bar{a}): \overline{\mathbf{C}_{G}(a)}\right|=q
$$

donde $\left|\mathbf{C}_{\bar{G}}(\bar{a}): \overline{\mathbf{C}_{G}(a)}\right| \neq 1$. Por tanto, $\left|\overline{\mathbf{N}_{G}\left(\mathbf{C}_{G}(a)\right)}: \mathbf{C}_{\bar{G}}(\bar{a})\right|=1$, y así, se tiene que $\left|\overline{\mathbf{N}_{G}\left(\mathbf{C}_{G}(a)\right)}\right|=\left|\mathbf{C}_{\bar{G}}(\bar{a})\right|$. Aplicando ahora el Paso 12 se concluye que $\left|\bar{G}: \mathbf{C}_{\bar{G}}(\bar{a})\right|=\left|G: \mathbf{N}_{G}\left(\mathbf{C}_{G}(a)\right)\right|$ es potencia de $p$. Entonces, por el Teorema 2.15, el grupo $\left\langle\bar{a}^{\bar{G}}\right\rangle$ es subgrupo normal resoluble de $\bar{G}$, y por consiguiente, existe algún primo $r$ tal que $\mathbf{O}_{r}(\bar{G}) \neq 1$. Pero si $r=p$, llegamos a una contradicción con el Paso 12, y si $r \neq p$, llegamos a una contradicción con el Paso 13.

Concluimos por tanto que $\bar{G}$ tiene dos tamaños de clases de conjugación de elementos $p$-regulares, y por inducción $\bar{G}$ tiene $p$-complemento abeliano o bien $\bar{G}=\overline{P Q} \times \bar{A}$, con $\bar{P} \in \operatorname{Syl}_{p}(\bar{G}), \bar{Q} \in \operatorname{Syl}_{q}(\bar{G})$ y $\bar{A} \subseteq \mathbf{Z}(\bar{G})$. En el primer caso, aplicando el Lema 3.2 se obtiene que todos los tamaños de clase de conjugación en $\overline{G_{p^{\prime}}}$ son potencias de $p$, y como $\left|\overline{\mathbf{C}_{G}(a)}\right|=\left|\mathbf{C}_{\bar{G}}(\bar{a})\right|$, todos los tamaños de clase de conjugación en $G_{p^{\prime}}$ son potencias de $p$ también, concluyéndose que $G$ tiene $p$ complemento abeliano. En el segundo caso se obtiene que $\bar{G}$ es resoluble y por tanto $G$ es resoluble, por lo que el teorema está demostrado.

Como consecuencia del resultado se tiene que si $\{1, m\}$ son los tamaños de clase de conjugación de elementos $p$-regulares de $G$ y $a \in G_{p^{\prime}}$, entonces 


$$
m=\left|G: \mathbf{C}_{G}(a)\right|=\left|G: \mathbf{N}_{G}\left(\mathbf{C}_{G}(a)\right)\right|\left|\mathbf{N}_{G}\left(\mathbf{C}_{G}(a)\right): \mathbf{C}_{G}(a)\right|=p^{a} q^{b} .
$$

En particular, si $b=0$ entonces $m=p^{a}$, y por el Lema 3.2, $G$ tiene $p$-complemento abeliano. Si $a=0$ entonces $m=q^{b}$, y aplicando el Lema 3.3 se concluye que $G=P \times H=P \times Q \times A$, siendo $H=Q \times A$ un $p$-complemento de $G$ y $A \in \mathbf{Z}(\bar{G})$.

Obsérvese que en todos los casos se concluye que el grupo $G$ es resoluble. Si se obtiene que $G$ es producto de un $p$-grupo por un $p$-complemento abeliano, la resolubilidad se tiene por el Teorema $p^{a} q^{b}$ de Burnside. Igualmente, si se obtiene que $G$ es producto directo de un $\{p, q\}$-grupo por un subgrupo abeliano, la resolubilidad resulta de aplicar el Teorema $p^{a} q^{b}$ de Burnside. 



\section{Capítulo 4}

\section{Grupos con dos tamaños de clases de elementos de orden potencia de primo}

\subsection{Introducción}

En este capítulo se estudia la relación entre la estructura de los grupos finitos y los tamaños de clases de conjugación de sus elementos de orden potencia de primo, o primarios. Esta relación nace del hecho de que los tamaños de clases de los elementos compuestos de un grupo son múltiplos de los tamaños de clases de los elementos de orden potencia de primo. Sin embargo, el conjunto de tamaños de clases de elementos de orden potencia de primo no coincide necesariamente con el conjunto de tamaños de clases del grupo. Es fácil comprobar que existen grupos para los que estos dos conjuntos difieren.

En el año 1996, L. Shirong demuestra que si el grupo finito $G$ tiene exactamente dos tamaños de clases de elementos de orden potencia de primo, entonces es resoluble (véase el teorema principal de [62]). Su demostración se apoya en la clasificación de grupos simples finitos y en el teorema de Feit-Thompson sobre la resolubilidad de los grupos de orden impar. En este capítulo obtenemos una mejora del resultado de Shirong, al tiempo que generalizamos el Teorema 3.1, considerando los tamaños de clases de conjugación de los elementos del grupo de orden potencia de primo, que es, probablemente, el conjunto más reducido que se puede considerar. Obsérvese que si debilitamos la hipótesis del teorema, trabajando sólo con los elementos de orden primo, entonces la tesis del Teorema 3.1 es falsa. Considérese, por ejemplo, un grupo $G$ definido como producto semidirecto del grupo cuaternio de orden 8, sobre el que actúa no trivialmente, un grupo cíclico de orden 3 . En este caso, todos los elementos 
de orden primo, es decir, de orden 2 ó 3, tienen tamaño de clase 1 ó 6 . Sin embargo, trivialmente $G$ no es nilpotente.

En el resultado principal del capítulo se demuestra que si $G$ es un grupo resoluble con dos tamaños de clases de conjugación de $p^{\prime}$-elementos de orden potencia de primo, entonces $G$ tiene $p$-complemento abeliano o el $p$-complemento, salvo factores centrales, es un $q$-grupo, con $q$ un primo distinto de $p$. Como corolario, y aplicando un reciente resultado de X. Zhao y X. Guo ([66]), que establece la resolubilidad de los grupos $p$-resolubles con dichos tamaños de clases, se obtiene la estrutura de los grupos $p$-resolubles con dos tamaños de clases de $p^{\prime}$-elementos de orden potencia de primo.

Aplicando la caracterización obtenida, hemos podido mejorar el resultado de Shirong sobre la resolubilidad de los grupos con dos tamaños de clases de elementos de orden potencia de primo. Partiendo de la resolubilidad, se demuestra que tales grupos son $p$-grupos, salvo factores centrales, y por tanto, nilpotentes, obteniéndose así una nueva extensión del resultado de Itô, al considerar los tamaños de clase de los elementos primarios.

\subsection{Resultados preliminares}

Para la obtención del resultado principal de este capítulo necesitaremos algunos resultados que exponemos a continuación. El primero de ellos es un resultado sencillo, poco conocido.

Lema 4.1 Sea $G$ un grupo finito tal que $G=A B, A \unlhd G, B \leq G$ y $A \cap B=1$. Si $x \in B$ entonces $\mathbf{C}_{G}(x)=\mathbf{C}_{A}(x) \mathbf{C}_{B}(x)$.

Demostración. Como $\mathbf{C}_{A}(x) \leq G_{G}(x)$ y $\mathbf{C}_{B}(x) \leq G_{G}(x)$, es trivial ver que $\mathbf{C}_{A}(x) \mathbf{C}_{B}(x) \subseteq G_{G}(x)$. Demostraremos por tanto, la otra inclusión.

Por las hipótesis del lema, para todo $g \in \mathbf{C}_{G}(x)$ se tiene que $g=a b$, con $a \in A \mathrm{y}$ $b \in B$. Entonces $g^{x}=(a b)^{x}=a^{x} b^{x}=a b$. Esto implica que $a^{-1} a^{x}=b\left(b^{x}\right)^{-1}$. Ahora bien, $a^{-1} a^{x} \in A$ y $b\left(b^{x}\right)^{-1} \in B$, por lo que $a^{-1} a^{x}=b\left(b^{x}\right)^{-1}=1$, por ser $A \cap B=1$. Es decir, $a^{x}=a$ y $b^{x}=b$, luego $a \in \mathbf{C}_{A}(x)$ y $b \in \mathbf{C}_{B}(x)$.

El siguiente lema proporciona dos resultados sobre tamaños de clases de conjugación en grupos $\pi$-separables. El primer resultado es una extensión de un teorema de A. Camina y R. Camina (consecuencia inmediata de la Proposición 1 de [23]) 
que establece que en un grupo $G$, cualquier elemento con índice $p^{a}$ se encuentra en $\mathbf{O}_{p, p^{\prime}}(G)$. En el apartado $a$ del lema se obtiene una generalización de este resultado a un conjunto arbitrario de primos $\pi$, y para un grupo $\pi$-separable. El apartado $b$ del lema es, a su vez, una generalización del Lema 3.2, para un conjunto de primos $\pi$ y un grupo $\pi$-separable. Queremos hacer notar que en ambos casos, si $\pi=\{p\}$, no se necesita la condición de $p$-resolubilidad del grupo.

Lema 4.2 Sea $G$ un grupo finito $\pi$-separable.

a) Si $x \in G$ y $\left|x^{G}\right|$ es un $\pi$-número, entonces $\left[x^{G}, x^{G}\right] \subseteq \mathbf{O}_{\pi}(G)$ y por tanto $x \in$ $\mathrm{O}_{\pi, \pi^{\prime}}(G)$.

b) El tamaño de clase de conjugación de cualquier $\pi^{\prime}$-elemento de $G$ es un $\pi$ número si y sólo si $G$ tiene $\pi^{\prime}$-subgrupos de Hall abelianos.

Demostración. a) Veamos que si $\left|x^{G}\right|$ es $\pi$-número entonces $\left[x^{G}, x^{G}\right] \subseteq \mathbf{O}_{\pi}(G)$.

Veremos primero que si $\mathbf{O}_{\pi}(G) \neq 1$ se tiene el resultado. Sea $\bar{G}=G / \mathbf{O}_{\pi}(G)$. Si $x \in G$ y $\left|x^{G}\right|$ es un $\pi$-número entonces $\left|\bar{x}^{\bar{G}}\right|$ es un $\pi$-número y $|\bar{G}|<|G|$, procediendo por inducción sobre el orden del grupo se tiene que:

$$
\left[\bar{x}^{\bar{G}}, \bar{x}^{\bar{G}}\right] \subseteq \mathbf{O}_{\pi}(\bar{G})=1 .
$$

Por lo tanto $\left[x^{G}, x^{G}\right] \subseteq \mathbf{O}_{\pi}(G)$. Como además, por inducción, $\bar{x} \in \mathbf{O}_{\pi, \pi^{\prime}}(\bar{G})$, entonces

$$
\bar{x} \in \mathbf{O}_{\pi^{\prime}}\left(\frac{\bar{G}}{\mathbf{O}_{\pi}(\bar{G})}\right)=\mathbf{O}_{\pi^{\prime}}(\bar{G})=\frac{\mathbf{O}_{\pi, \pi^{\prime}}(G)}{\mathbf{O}_{\pi}(G)}=\overline{\mathbf{O}_{\pi, \pi^{\prime}}(G)}
$$

y por tanto $x \in \mathbf{O}_{\pi, \pi^{\prime}}(G)$.

Podemos suponer por tanto que $\mathbf{O}_{\pi}(G)=1$ y demostraremos que $\left[x^{G}, x^{G}\right]=1$. Sea $N=\left\langle x^{G}\right\rangle$ y nótese que $\mathbf{O}_{\pi}(N) \subseteq \mathbf{O}_{\pi}(G)=1$. Supongamos que $N<G$. Como $N$ es subgrupo $\pi$-separable de $G$, podemos aplicar inducción y concluir que $\left[x^{N}, x^{N}\right] \subseteq \mathbf{O}_{\pi}(N)=1$, es decir $\left[x^{N}, x^{N}\right]=1$. Por tanto $\left\langle x^{N}\right\rangle$ es un subgrupo subnormal abeliano de $G$, y por tanto nilpotente. Concluimos que $x \in\left\langle x^{N}\right\rangle \subseteq \mathbf{F}(G)$, luego $\left\langle x^{G}\right\rangle \subseteq \mathbf{F}(G)$. Pero $\mathbf{F}(G) \subseteq \mathbf{O}_{\pi}(G) \mathbf{O}_{\pi^{\prime}}(G)=\mathbf{O}_{\pi^{\prime}}(G)$ es $\pi^{\prime}$-grupo, por lo que $\mathbf{F}(G) \subseteq \mathbf{C}_{G}(x)$ y por tanto $\left\langle x^{G}\right\rangle \subseteq \mathbf{Z}(\mathbf{F}(G))$. Como $\left\langle x^{G}\right\rangle$ es central en $\mathbf{F}(G)$, el conmutador $\left[x^{G}, x^{G}\right]=1$.

Podemos asumir por tanto que $N=G$. Consideremos un subgrupo $K$ normal minimal de $G$. Como $K$ es $\pi$-separable y $\mathbf{O}_{\pi}(K)=1$, será $\mathbf{O}_{\pi^{\prime}}(K) \neq 1$, y por ser 
subgrupo normal minimal de $G$ se tiene que $\mathbf{O}_{\pi^{\prime}}(K)=K$ y así $K$ es $\pi^{\prime}$-grupo. Por tanto $K \subseteq \mathbf{C}_{G}(x)$, y así $K \subseteq \mathbf{C}_{G}\left(x^{g}\right)$, para todo $g \in G$, obteniéndose que $K \subseteq \mathbf{Z}(G)$. Veremos que $\mathbf{O}_{\pi}(G / K)=1$. Escribimos $\mathbf{O}_{\pi}(G / K)=H / K$, con $K \leq H \leq G$. Se sigue que $H / K$ es $\pi$-subgrupo normal del cociente, por lo que $|H: K|$ es un $\pi$ número y $|K|$ es $\pi^{\prime}$-número, donde $K$ es central en $G$ y, por tanto, subgrupo normal abeliano de $H$. En consecuencia, existe complemento normal $L$ de $K$ en $H$. Podemos poner, por tanto, $H=L \times K$, con $L$ un $\pi$-subgrupo normal de $H$. Pero entonces $L \leq \mathbf{O}_{\pi}(H) \leq \mathbf{O}_{\pi}(G)=1$ y se obtiene que $L=\mathbf{O}_{\pi}(G)$ y por tanto $\mathbf{O}_{\pi}(G / K)=1$.

Sea $\bar{G}=G / K$. Como $\bar{x} \in \bar{G}$ verifica que $\left|\bar{x}^{\bar{G}}\right|$ es un $\pi$-número, por inducción se tiene que $\left[\bar{x}^{\bar{G}}, \bar{x}^{\bar{G}}\right]=1$. Es decir $\left[x^{G}, x^{G}\right] \subseteq K \leq \mathbf{Z}(G)$, por lo que $\left\langle x^{G}\right\rangle^{\prime}=G^{\prime} \subseteq \mathbf{Z}(G)$ y $G$ es nilpotente. Por consiguiente, $G=\mathbf{O}_{\pi}(G) \times \mathbf{O}_{\pi^{\prime}}(G)=\mathbf{O}_{\pi^{\prime}}(G)$ es $\pi^{\prime}$-grupo. Como $\left|x^{G}\right|$ es $\pi$-número, entonces $\left|x^{G}\right|=1$. Es decir, $x$ es central en $G$ y $\left[x^{G}, x^{G}\right]=1$.

Veamos ahora que $x \in \mathbf{O}_{\pi, \pi^{\prime}}(G)$. Notar que

$$
\frac{\left\langle x^{G}\right\rangle \mathbf{O}_{\pi}(G)}{\mathbf{O}_{\pi}(G)} \subseteq F\left(\frac{G}{\mathbf{O}_{\pi}(G)}\right) \subseteq \mathbf{O}_{\pi^{\prime}}\left(\frac{G}{\mathbf{O}_{\pi}(G)}\right)=\frac{\mathbf{O}_{\pi, \pi^{\prime}}(G)}{\mathbf{O}_{\pi}(G)} .
$$

Por tanto $\left\langle x^{G}\right\rangle \mathbf{O}_{\pi}(G) \subseteq \mathbf{O}_{\pi, \pi^{\prime}}(G)$ y $x \in \mathbf{O}_{\pi, \pi^{\prime}}(G)$, como se quería demostrar.

b) Es fácil ver que si $G$ tiene $\pi$-complementos abelianos, entonces el tamaño de clase de conjugación de cada $\pi^{\prime}$-elemento $x$ de $G$ es $\pi$-número. Obsérvese que, como $G$ es $\pi$-separable, si $H$ es un $\pi$-complemento de $G$ entonces $\langle x\rangle \subseteq H^{g}$, para un cierto elemento $g \in G$, por lo que $H^{g} \leq \mathbf{C}_{G}(x)$ y el índice de $\mathbf{C}_{G}(x)$ en $G$ es $\pi$-número.

Supongamos por tanto que $\left|x^{G}\right|$ es $\pi$-número para todo elemento $x \in G_{\pi^{\prime}}$. Trabajaremos por inducción sobre $|G|$ para obtener el resultado. Si $\mathbf{O}_{\pi}(G) \neq 1$, consideramos $\bar{G}=G / \mathbf{O}_{\pi}(G)$. Como el grupo cociente verifica las hipótesis del teorema, se concluye por inducción que $\bar{G}$ tiene $\pi$-complementos abelianos de la forma $\bar{H}=H / \mathbf{O}_{\pi}(G)$, con $\mathbf{O}_{\pi}(G) \leq H \leq G$. Por lo tanto, podemos poner $H=H_{1} \mathbf{O}_{\pi}(G)$, siendo $H_{1}$ un $\pi$-complemento de $H$. Entonces

$$
\bar{H}=\frac{H}{\mathbf{O}_{\pi}(G)}=\frac{H_{1} \mathbf{O}_{\pi}(G)}{\mathbf{O}_{\pi}(G)} \cong \frac{H_{1}}{H_{1} \cap \mathbf{O}_{\pi}(G)}=H_{1}
$$

y se tiene que $H_{1}$ es $\pi$-complemento abeliano de $G$.

Supongamos ahora que $\mathbf{O}_{\pi}(G)=1$. De las hipótesis del teorema se deduce que para todo $x \in G_{\pi^{\prime}}$ existe al menos un $\pi$-complemento $H$ de $G$ contenido en $\mathbf{C}_{G}(x)$, por lo que $\mathbf{O}_{\pi^{\prime}}(G) \subseteq H$. Como $\mathbf{O}_{\pi}(G)=1$, entonces $\mathbf{C}_{G}\left(\mathbf{O}_{\pi^{\prime}}(G)\right) \subseteq \mathbf{O}_{\pi^{\prime}}(G)$ y, por 
tanto,

$$
x \in \mathbf{C}_{G}(H) \subseteq \mathbf{C}_{G}\left(\mathbf{O}_{\pi^{\prime}}(G)\right) \subseteq \mathbf{O}_{\pi^{\prime}}(G) \subseteq H
$$

obteniéndose que $H=\mathbf{O}_{\pi^{\prime}}(G)$ y que $H$ es abeliano.

Enunciamos a continuación el resultado de Shirong sobre la resolubilidad de los grupos finitos con dos tamaños de clases de elementos de orden potencia de primo.

Teorema 4.3 Si el grupo finito $G$ tiene exactamente dos tamaños de clases de elementos de orden potencia de primo, entonces $G$ es resoluble.

Demostración. Véase el resultado principal de [62].

Siguiendo un esquema similar al de la demostración del teorema anterior, Zhao y Guo hacen un primer intento por determinar la estructura de un grupo a partir de los tamaños de clases de sus $p^{\prime}$-elementos de orden potencia de primo, obteniendo el resultado que enunciamos a continuación.

Teorema 4.4 Sea $G$ un grupo finito p-resoluble y $m$ un entero positivo. Si $G$ tiene dos tamaños de clase de conjugación, 1 ó $m$, de p'-elementos de orden potencia de primo, entonces $G$ es resoluble.

Demostración. Véase el resultado principal de [66].

\subsection{Resultados Principales}

A continuación presentamos los resultados principales del capítulo. El primer resultado es una generalización del Teorema de Itô para grupos resolubles con dos tamaños de clases de conjugación de $p^{\prime}$-elementos de orden potencia de primo. La dificultad de la demostración se debe a que sólo se trabaja con los $p^{\prime}$-elementos del grupo, y ello produce una pérdida de información sobre la $p$-estructura del grupo.

Teorema 4.5 Sea $G$ un grupo p-resoluble y supongamos que $G$ tiene exactamente dos tamaños de clases de conjugación de $p^{\prime}$-elementos de orden potencia de primo, 1 y $m$. Entonces $m=p^{a} q^{b}$, con $q$ un primo distinto de $p$, y $a, b \geq 0$. Si $b=0$ entonces $G$ tiene $p$-complementos abelianos. Si $b \neq 0$ entonces $G=P Q \times A$, con $P$ un p-subgrupo de Sylow de $G, Q$ un q-subgrupo de Sylow de $G$ y $A \subseteq \mathbf{Z}(G)$. 
Demostración. Aplicando el Teorema 4.4 el grupo es resoluble. Procederemos por inducción sobre el orden del grupo. Veamos a continuación que podemos suponer que todo primo $r \neq p$ divisor de $|G|$ también divide a $m$.

Supongamos que existe un primo $r \neq p$ divisor de $|G|$ que no divide a $m$. Entonces para cada $x \in G_{p^{\prime}}$ de orden potencia de primo, $r$ no divide a $\left|x^{G}\right|=\left|G: \mathbf{C}_{G}(x)\right| \mathrm{y}$ por tanto $\left|G: \mathbf{C}_{G}(x)\right|$ es $r^{\prime}$-número. Aplicando el Lema 4.2 se tiene que $x \in \mathbf{O}_{r^{\prime}, r}(G)$. Si $x \in G_{p^{\prime}}$ tiene orden compuesto, consideramos su descomposición como producto de elementos de orden potencia de primo, $x=x_{q_{1}} x_{q_{2}} \ldots x_{q_{t}}$, con cada $x_{q_{i}} \in \mathbf{O}_{r^{\prime}, r}(G)$. Luego $x \in \mathbf{O}_{r^{\prime}, r}(G)$ y $G_{p^{\prime}} \subseteq \mathbf{O}_{r^{\prime}, r}(G)$, es $\operatorname{decir}, G / \mathbf{O}_{r^{\prime}, r}(G)$ es $p$-grupo.

Sea $P$ un $p$-subgrupo de Sylow de $G$. Como $\left(\left|G: \mathbf{O}_{r^{\prime}, r}(G)\right|,|G: P|\right)=1$ se tiene que $G=P \mathbf{O}_{r^{\prime}, r}(G)$. Si $\mathbf{O}_{r^{\prime}}(G) \subseteq \mathbf{Z}(G)$ entonces $\mathbf{O}_{r^{\prime}, r}(G)=R \times \mathbf{Z}(G)_{r^{\prime}}$, y por tanto $G=P R \times \mathbf{Z}(G)_{\{p, r\}^{\prime}}$.

Sea $K=P \mathbf{O}_{r^{\prime}}(G)$, con $\mathbf{O}_{r^{\prime}}(G)$ no central. Si $y \in K$ es un elemento $p$-regular, no central de $G$, de orden potencia de primo, entonces $\left|G: \mathbf{C}_{G}(y)\right|$ es $r^{\prime}$-número. Por otro lado, $|G: K|$ es potencia de $r$ y se tiene que $\left(\left|G: \mathbf{C}_{G}(y)\right|,|G: K|\right)=1$ luego $G=\mathbf{C}_{G}(y) K$. Entonces

$$
\left|y^{G}\right|=\frac{|G|}{\left|\mathbf{C}_{G}(y)\right|}=\frac{\left|\mathbf{C}_{G}(y) K\right|}{\left|\mathbf{C}_{G}(y)\right|}=\frac{|K|}{\left|\mathbf{C}_{K}(y)\right|}=\left|y^{K}\right|=m .
$$

Podemos aplicar inducción sobre el orden del grupo y concluir que $m=p^{a}$ y $K$ tiene $p$-complementos abelianos ó $m=p^{a} q^{b}$, para algún primo $q \neq p, b>0 \mathrm{y}$ $K=P Q \times A$, con $P$ un $p$-subgrupo de Sylow de $K, Q$ un $q$-subgrupo de Sylow de $K$ y $A$ abeliano.

Supongamos primero que $m=p^{a}$ y que $K$ tiene $p$-complementos abelianos. Veamos que los $p$-complementos de $G$ son abelianos también. Si $z$ es un elemento no central de $G_{p^{\prime}} \backslash K$ de orden potencia de primo, entonces $\left|z^{G}\right|=m=p^{a}$, y por el Lema 4.2, se tiene que $z \in \mathbf{O}_{p, p^{\prime}}(G)$. Si $x \in G_{p^{\prime}}$ es de orden compuesto, considerando su factorización en producto de elementos $p$-regulares de orden potencia de primo, $x=$ $x_{q_{1}} x_{q_{2}} \ldots x_{q_{t}}$, se concluye que los factores están en $\mathbf{O}_{p, p^{\prime}}(G)$, por lo que $x \in \mathbf{O}_{p, p^{\prime}}(G)$ y $G_{p^{\prime}} \subseteq \mathbf{O}_{p, p^{\prime}}(G)$. Sea $N=\mathbf{O}_{p, p^{\prime}}(G)$, entonces si $H$ es un $p$-complemento de $G$ se sigue que $N=\mathbf{O}_{p}(G) H$. Sea $x \in H$ un elemento de orden potencia de primo. Como $N=\mathbf{O}_{p}(G) H$, por el Lema 4.1 se tiene que $\mathbf{C}_{N}(x)=\mathbf{C}_{\mathbf{O}_{p}(G)}(x) \mathbf{C}_{H}(x)$, de donde

$$
\begin{aligned}
\left|N: \mathbf{C}_{N}(x)\right| & =\frac{|N|}{\left|\mathbf{C}_{N}(x)\right|}=\frac{\left|\mathbf{O}_{p}(G) H\right|}{\left|\mathbf{C}_{\mathbf{O}_{p}(G)}(x)\right|\left|\mathbf{C}_{H}(x)\right|} \\
& =\left|\mathbf{O}_{p}(G): \mathbf{C}_{\mathbf{O}_{p}(G)}(x)\right|\left|H: \mathbf{C}_{H}(x)\right| .
\end{aligned}
$$


Por tanto,

$$
\begin{aligned}
\mid G & : \mathbf{C}_{G}(x)|| \mathbf{C}_{G}(x): \mathbf{C}_{N}(x)|=| G: N|| N: \mathbf{C}_{N}(x) \mid \\
& =|G: N|\left|\mathbf{O}_{p}(G): \mathbf{C}_{\mathbf{O}_{p}(G)}(x)\right|\left|H: \mathbf{C}_{H}(x)\right| .
\end{aligned}
$$

Obsérvese que $\left|G: \mathbf{C}_{G}(x)\right|\left|\mathbf{C}_{G}(x): \mathbf{C}_{N}(x)\right|$ es producto de potencias de $p$, ya que $\left|G: \mathbf{C}_{G}(x)\right|=p^{a}$ y, por otro lado, $\left|\mathbf{C}_{G}(x): \mathbf{C}_{N}(x)\right|=\left|\mathbf{C}_{G}(x) N: N\right|$ divide a $|G: N|$, siendo este último índice una potencia de $p$. Por tanto $\left|H: \mathbf{C}_{H}(x)\right|=1$. Es decir, $x \in \mathbf{Z}(H)$. Por consiguiente, todo elemento de $H$ está en $\mathbf{Z}(H)$ y concluimos que $H$ es abeliano.

Supongamos ahora que $K=P Q \times A$ y $m=p^{a} q^{b}$, con $P$ un $p$-subgrupo de Sylow de $K, Q$ un $q$-subgrupo de Sylow de $K$ y $A$ abeliano. Como $|G: K|$ es potencia de $r$ se tiene que $P$ es $p$-subgrupo de Sylow de $G$. Además $\left|\mathbf{O}_{r^{\prime}, r}(G): \mathbf{O}_{r^{\prime}}(G)\right|$ es potencia de $r$ también, por lo que

$$
|G|_{r^{\prime}}=\left|\mathbf{O}_{r^{\prime}, r}(G)\right|_{r^{\prime}}=\left|\mathbf{O}_{r^{\prime}}(G)\right|=|K|_{r^{\prime}}
$$

y en particular $|G|_{q}=|K|_{q}$, y por tanto $Q$ es $q$-subgrupo de Sylow de $G$. Veamos ahora que $A \subseteq \mathbf{Z}(G)$. Supongamos que existe un elemento de $A$, no central en $G$. Considerando su descomposición en producto de elementos de orden potencia de primo se concluye que existe algún elemento $w \in A \backslash \mathbf{Z}(G)$, de orden potencia de primo. Como $A$ centraliza $Q$, se tiene que $Q \subseteq \mathbf{C}_{K}(w)$ y el primo $q$ no divide a $\left|G: \mathbf{C}_{G}(w)\right|$. Pero, por la hipótesis de inducción se tiene que $m=\left|K: \mathbf{C}_{K}(w)\right|=p^{a} q^{b}=\left|G: \mathbf{C}_{G}(w)\right|, \mathrm{y}$ obtenemos una contradicción. Concluimos que los únicos elementos de $K$ no centrales en $G$ son $\{p, q\}$-elementos y los únicos elementos no centrales de $G$ son $\{p, q, r\}$ elementos, por lo que podemos asumir que $K$ es un $\{p, q\}$-grupo y $G$ un $\{p, q, r\}$ grupo. Por tanto, $K=P Q$, con $Q$ un $q$-subgrupo de Sylow de $G$ y $\mathbf{O}_{r^{\prime}}(G)=P_{0} Q$, con $P_{0}$ un $p$-subgrupo de $G$. Obsérvese que si $H$ es un $p$-complemento de $G$, de forma que $H=R Q$ se tiene que $Q=H \cap \mathbf{O}_{r^{\prime}}(G) \unlhd H$. Y concluimos que todo $p$-complemento de $G$ tiene un $q$-subgrupo de Sylow normal.

Por la hipótesis inicial, podemos suponer que existe un $r$-elemento no central $x$ de $G$. Sea $Q_{0}$ un $q$-subgrupo de Sylow de $\mathbf{C}_{G}(x)$, entonces $\left\langle Q_{0}, x\right\rangle$ es un $p^{\prime}$-grupo de $G$ y por tanto está contenido en algún $p$-complemento $H \subseteq G$. Sea $Q$ el $q$-subgrupo de Sylow de $G$ que es normal en $H$. Se tiene que $B=Q_{0} \times\langle x\rangle$ normaliza a $Q$ y por tanto $B$ actúa sobre $Q$. Veamos que $\mathbf{C}_{Q}\left(Q_{0}\right) \subseteq Q_{0}$. Si $z \in \mathbf{C}_{Q}\left(Q_{0}\right)$ es central en $G$ entonces, trivialmente, $z \in Q_{0}$. Si $z$ no es central en $G$, como $Q_{0} \subseteq \mathbf{C}_{G}(z)$, se tiene que $\left\langle Q_{0}, z\right\rangle \subseteq \mathbf{C}_{G}(z)$, y como $\left|\mathbf{C}_{G}(z)\right|_{q}=\left|\mathbf{C}_{G}(x)\right|_{q}=\left|Q_{0}\right|$, se sigue que $\left|\left\langle Q_{0}, z\right\rangle\right|=\left|Q_{0}\right|$ y $z \in Q_{0}$. Podemos, por tanto, aplicar el Teorema 2.10 y concluir que $Q \subseteq \mathbf{C}_{G}(x)$, lo que contradice el hecho de que $q$ divide a $m$. Por tanto, podemos suponer que todo primo $r \neq p$ divisor de $|G|$ también divide a $m$, como se quería 
probar.

Denotemos por $\bar{G}=G / \mathbf{O}_{p}(G)$ y usaremos barras para trabajar en el grupo cociente. Asumiremos que $\bar{G}$ no es abeliano, ya que en tal caso $G$ tiene $p$-complemento abeliano y el teorema se verifica. Sea $x$ un $p^{\prime}$-elemento no central en $G$ y de orden potencia de primo. Tenemos que $\mathbf{C}_{\bar{G}}(\bar{x})=H_{0} P_{0} / \mathbf{O}_{p}(G)$, con $H_{0}$ un $p$-complemento de $H_{0} P_{0}$ y $x \in H_{0}$. Si $y \in H_{0}$, entonces $\bar{y} \in \mathbf{C}_{\bar{G}}(\bar{x})$. Luego $[x, y] \in H_{0} \cap \mathbf{O}_{p}(G)=1$, es decir $y \in \mathbf{C}_{G}(x)$. Esto implica que

$$
H_{0} \subseteq \mathbf{C}_{G}(x) \subseteq H_{0} P_{0} \subseteq G .
$$

Luego $\left|\bar{G}: \mathbf{C}_{\bar{G}}(\bar{x})\right|_{p^{\prime}}=\left|G: H_{0} P_{0}\right|_{p^{\prime}}=m_{p^{\prime}}$ y así $\left|\mathbf{C}_{\bar{G}}(\bar{x})\right|_{p^{\prime}}=|\bar{G}|_{p^{\prime}} / m_{p^{\prime}}$ es constante. Veamos que $\overline{\mathbf{Z}(G)_{p^{\prime}}}=\mathbf{Z}(\bar{G})_{p^{\prime}}$. La inclusión directa es evidente. Supongamos pues que $\bar{x} \in \mathbf{Z}(\bar{G})_{p^{\prime}}$ es un elemento de orden potencia de un cierto primo $s$. Considerando la descomposición de $x=x_{s} x_{s^{\prime}}$, en su $s$-parte y $s^{\prime}$-parte respectivamente, entonces $\bar{x}=\overline{x_{s}}$; es decir, que podemos suponer que $x$ es un $s$-elemento. Entonces si $x \notin \mathbf{Z}(G)$, podemos aplicar la igualdad anterior y se obtiene que $1=\left|\bar{G}: \mathbf{C}_{\bar{G}}(\bar{x})\right|_{p^{\prime}}=m_{p^{\prime}}, \mathrm{y}$ por tanto, $m$ sería una potencia de $p$, lo cual es una contradicción. Luego $x \in \mathbf{Z}(G)_{p^{\prime}}$ y se tiene la otra inclusión.

Ya que $\bar{G}$ es resoluble no abeliano, debe existir un primo $q \neq p$ tal que $\mathbf{Z}(\bar{G})_{q}<$ $\mathrm{O}_{q}(\bar{G})$. Si no existe ningún $r$-elemento, con $r$ distinto de $p$ y $q$, no central en $\bar{G}$, entonces el índice $|\bar{G}: \mathbf{Z}(\bar{G})|$ es un $\{p, q\}$-número y, por el párrafo anterior, se sigue que el índice $|G: \mathbf{Z}(G)|$ es también un $\{p, q\}$-número. En consecuencia, $G=P Q \times A$, con $P$ un $p$-subgrupo de Sylow de $G, Q$ un $q$-subgrupo de Sylow de $G$ y $A$ un $\{p, q\}^{\prime}$ subgrupo abeliano, y el teorema está probado.

Suponemos pues que existe un $r$-elemento $\bar{w}$, con $r$ distinto de $p$ y $q$, no central en $\bar{G}$. Veremos que esta situación no puede darse. Como antes, se puede asumir que $w$ es un $r$-elemento no central en $G$. Sea $\overline{Q_{0}}$ un $q$-subgrupo de Sylow de $\mathbf{C}_{\bar{G}}(\bar{w})$. Consideramos la acción de $\overline{Q_{0}} \times\langle\bar{w}\rangle$ sobre $\bar{Q}:=\mathbf{O}_{q}(\bar{G}) \overline{Q_{0}}$. Veamos que $\mathbf{C}_{\bar{Q}}\left(\overline{Q_{0}}\right) \subseteq \overline{Q_{0}}$. Sea $\bar{z} \in \mathbf{C}_{\bar{Q}}\left(\overline{Q_{0}}\right)$. Si $\bar{z}$ es un $q$-elemento central en $\bar{G}$, entonces trivialmente está en $\overline{Q_{0}}$. Luego podemos considerar que $\bar{z}$ es no central en $\bar{G}$ y, por tanto, podemos suponer que $z$ es un $q$-elemento no central en $G$. Tenemos que $\left\langle\overline{Q_{0}}, \bar{z}\right\rangle \subseteq \mathbf{C}_{\bar{G}}(\bar{z})$; $\sin$ embargo, como hemos visto en el párrafo anterior,

$$
\left|\mathbf{C}_{\bar{G}}(\bar{z})\right|_{q}=|\bar{G}|_{q} / m_{q}=\left|\mathbf{C}_{\bar{G}}(\bar{w})\right|_{q}=\left|\overline{Q_{0}}\right|,
$$

de donde se sigue que $\bar{z} \in \overline{Q_{0}}$. Aplicando el Teorema 2.10 tenemos que $\bar{w} \in \mathbf{C}_{\bar{G}}(\bar{Q})$. En particular, $\bar{w} \in \mathbf{C}_{\bar{G}}\left(\mathbf{O}_{q}(\bar{G})\right)$ para todo $r$-elemento $w$ de $G$. Por tanto, si $R$ es un $r$-subgrupo de Sylow de $G$, entonces $\overline{R \mathbf{O}_{p}(G)} \subseteq \mathbf{C}_{\bar{G}}\left(\mathbf{O}_{q}(\bar{G})\right)$. Por otro lado, sabemos 
que existe $\bar{t} \in \mathbf{O}_{q}(\bar{G}) \backslash \mathbf{Z}(\bar{G})$, con $t$ un $q$-elemento no central en $G$, luego $\overline{R \mathbf{O}_{p}(G)} \subseteq$ $\mathbf{C}_{\bar{G}}(\bar{t}) \subset \bar{G}$, lo cual es una contradicción pues $r$ divide a $m_{p^{\prime}}=\left|\bar{G}: \mathbf{C}_{\bar{G}}(\bar{t})\right|_{p^{\prime}}$.

Como caso particular, cuando el primo $p$ no divide al orden del grupo obtenemos una nueva extensión del Teorema 3.1 para grupos con dos tamaños de clases de elementos de orden potencia de primo. Obsérvese que en este caso la resolubilidad está garantizada por el Teorema 4.3.

Corolario 4.6 Sea $G$ un grupo finito y supongamos que $G$ tiene exactamente dos tamaños de clases de conjugación de elementos de orden potencia de primo, 1 y $m$. Entonces $m$ es potencia de un primo $p, y G$ es nilpotente. En consecuencia, $G=P \times A$, con $P \in \operatorname{Syl}_{p}(G)$ y $A \leq \mathbf{Z}(G)$.

Demostración. Por el Teorema 4.3, el grupo $G$ es resoluble. Consideremos un primo $p$ que no divida a $|G|$. Entonces todos los $p^{\prime}$-elementos de orden potencia de primo de $G$ tienen tamaños de clase 1 ó $m$. Aplicando el Teorema 4.5 se concluye que $m=q^{b}$, con $q$ un primo distinto de $p$, y $G=Q \times A$, siendo $Q$ un $q$-subgrupo de Sylow de $G$ y $A \leq \mathbf{Z}(G)$.

Queremos hacer notar, como consecuencia importante del Teorema 4.5, que si $G$ es un grupo $p$-resoluble con tamaños de clase de $p^{\prime}$-elementos de orden potencia de primo 1 ó $m$, entonces todos los $p^{\prime}$-elementos $G$ tienen tamaños de clase 1 ó $m$. De manera análoga, por el Corolario 4.6, si un grupo $G$ tiene dos tamaños de clase de elementos de orden potencia de primo, entonces $G$ tiene sólo dos tamaños de clases de todos sus elementos. 



\section{Capítulo 5}

\section{Subgrupos normales con dos tamaños de $G$-clases de conjugación}

\subsection{Introducción}

En este último capítulo extendemos y desarrollamos las metodologías y técnicas comentadas en capítulos anteriores sobre el análisis de la estructura de los grupos finitos a partir de los tamaños de clases, con el fin de obtener información sobre la estructura de los subgrupos normales del grupo a partir de los tamaños de sus $G$-clases de conjugación. Ya que los subgrupos normales son unión de clases de conjugación en $G$, es normal cuestionarse este tipo de información. Hemos de señalar que este es un campo poco investigado, siendo la bibliografía sobre el tema escasa en resultados.

Queremos hacer notar que las extensiones de resultados a subgrupos normales del grupo no son sencillas, ya que el cardinal del conjunto de tamaños de clases del subgrupo normal $N$ de $G$ no está necesariamente acotado por el cardinal del conjunto de tamaños de sus $G$-clases. Un ejemplo de ello es el siguiente: considérese el grupo pequeño $G$, con número $\operatorname{Id}(64,241)$, que se obtiene de la librería de grupos pequeños del programa GAP ([64]). El grupo $G$ tiene un subgrupo normal $N$ con número $\operatorname{Id}(32,33)$, tal que $c s(N)=\{1,2,4\}$. Sin embargo, los tamaños de $G$-clases contenidas en $N$ son $c s_{G}(N)=\{1,4\}$.

Como ya hemos comentado, Itô demuestra en 1953 que un grupo finito con todas sus clases de conjugación de elementos no centrales del mismo tamaño es nilpotente. Resulta natural preguntarse si los subgrupos normales de $G$ cuyos tamaños de $G$ clases son 1 ó $m$, para algún entero $m$, son a su vez nilpotentes, es decir, si tales subgrupos estan contenidos en el Fitting de $G$. Recientemente, X. Guo y X. Zhao ([34]) demuestran la nilpotencia de los subgrupos normales de $G$ con dos tamaños de $G$-clases que verifican la condición adicional de contener algún $p$-subgrupo de Sylow 
de $G$, para un cierto primo $p$. Sin embargo, esta hipótesis es muy restrictiva. El objetivo principal del capítulo es eliminar dicha condición de la hipótesis y demostrar que todo subgrupo normal $N$ de $G$, con dos tamaños de $G$-clases de conjugación, es nilpotente. Concretamente, demostraremos que $N$ es abeliano o $N$ es producto de un $p$-grupo por un subgrupo central en $G$.

La clave de la demostración es la utilización de propiedades estructurales de los CP-grupos o grupos cuyos elementos son todos de orden potencia de primo. La estructura de los CP-grupos resolubles fue investigada inicialmente por G. Higman en el año 1957 ([36]). Posteriormente, tras el desarrollo de los grupos simples finitos, H. Heineken ([35]) obtiene la clasificación los CP-grupos simples y estudia la estructura de los CP-grupos no resolubles. Nosotros haremos uso de la caracterización de los CP-grupos simples no abelianos y de las propiedades de los CP-grupos no resolubles, dadas por Heineken para concluir nuestra tesis.

Finalizaremos el capítulo extendiendo un conocido resultado sobre $p$-grupos que establece que si $G$ es un $p$-grupo con dos tamaños de clases de conjugación, entonces $G / \mathbf{Z}(G)$ tiene exponente $p$ (véase [41], [51] y [65])). Nosotros demostramos este resultado para un $p$-subgrupo normal $P$ de un grupo $G$, que contiene exactamente dos tamaños de $G$-clases.

\subsection{Resultados preliminares}

Con el fin de demostrar los resultados principales del capítulo hemos necesitado desarrollar algunos resultados preliminares que exponemos a continuación. El primero de ellos hace referencia al conjunto $c s_{\pi}(G)$ que es el conjunto de tamaños de clases de conjugación de los $\pi$-elementos de $G$.

Lema 5.1 Si $G$ es un grupo $\pi$-separable, entonces todo elemento de $\operatorname{cs}_{\pi}(G)$ es un $\pi$-número si y sólo si $G=H \times K$, siendo $H$ un $\pi$-subgrupo Hall de $G$ y $K$ un $\pi$-complemento de $G$.

Demostración. Como $G$ es $\pi$-separable existe un $\pi$-subgrupo Hall $H$ de $G$ y un $\pi^{\prime}$-subgrupo Hall $K$ de $G$. Además, $(|G: H|,|G: K|)=1$, por lo que $G=H K$, y $H \cap K=1$.

Veamos primero que si el tamaño de clase de conjugación de cada $\pi$-elemento de $G$ es un $\pi$-número entonces $G=H \times K$. Es suficiente demostrar que $[H, K]=1$. Sea $x \in G_{\pi}$. Como $\left|x^{G}\right|$ es $\pi$-número, se tiene que $|G|_{\pi^{\prime}}=\left|\mathbf{C}_{G}(x)\right|_{\pi^{\prime}}$, y por tanto, existe 
un $\pi$-complemento de $G$ contenido en $\mathbf{C}_{G}(x)$. Como los $\pi$-complementos son todos conjugados, existe un elemento $g \in G$ tal que $K^{g} \subseteq \mathbf{C}_{G}(x)$. Es decir, $x \in \mathbf{C}_{G}\left(K^{g}\right)$, lo que implica que

$$
H \subseteq \bigcup_{g \in G} \mathbf{C}_{G}\left(K^{g}\right) .
$$

Como $g=h k$, con $h \in H$ y $k \in K$, se tiene que

$$
H \subseteq \bigcup_{h \in H} \mathbf{C}_{G}\left(K^{h}\right) \cap H \subseteq \bigcup_{h \in H} \mathbf{C}_{H}(K)^{h} \subseteq H
$$

Se concluye que $H=\mathbf{C}_{H}(K)$, y por tanto, $K$ y $H$ conmutan.

El recíproco se verifica trivialmente, ya que si $G=K \times H$, entonces $K \subseteq \mathbf{C}_{G}(H)$ y $H \subseteq \mathbf{C}_{G}(K)$, por lo que si $x$ es un $\pi$-elemento de $G$, entonces $x \in H$ y $K \subseteq$ $\mathbf{C}_{G}(H) \subseteq \mathbf{C}_{G}(x)$, luego en particular $\left|G: \mathbf{C}_{G}(x)\right|$ es $\pi$-número.

En el siguiente lema demostramos que un grupo resoluble $G$, tal que todas las $G$-clases del $p$-radical son de orden potencia de $p$, para cada primo $p$, es nilpotente.

Lema 5.2 Sea $G$ un grupo resoluble finito tal que para cada primo $q$ y cada $x \in$ $\mathbf{O}_{q}(G)$ el indice $\left|G: \mathbf{C}_{G}(x)\right|$ es potencia de $q$. Entonces $G$ es nilpotente.

Demostración. Trabajaremos por inducción sobre el orden de $G$. Obsérvese que, por el Lema 2.4, cualquier subgrupo $N$ normal de $G$ verifica también las hipótesis del lema, y aplicando inducción se obtiene que cada subgrupo normal propio de $G$ es nilpotente.

Supongamos que $G$ no es nilpotente. Entonces $\mathbf{F}(G)$ es subgrupo normal maximal de $G$, por lo que $G / \mathbf{F}(G)$ es simple resoluble, y por tanto cíclico de orden primo. Se concluye que $|G: \mathbf{F}(G)|=p$, para algún primo $p$. Si $q \neq p$ es un primo divisor de $|\mathbf{F}(G)|$, entonces todo $q$-elemento $x \in G$ está en $\mathbf{F}(G)$, que, por ser nilpotente, es producto directo de sus radicales. Es decir, $x \in \mathbf{O}_{q}(\mathbf{F}(G)) \subseteq \mathbf{O}_{q}(G)$, y por tanto $\left|x^{G}\right|$ es potencia de $q$. Podemos aplicar ahora el Lema 5.1 y concluir que $G$ factoriza como producto de un $q$-subgrupo $Q$ de Sylow de $G$ por un $q$-complemento $H$ de $G$, esto es, $G=Q \times H$. Por tanto $Q, H \unlhd G$, y por la hipótesis de inducción se tiene que $Q$ y $H$ son nilpotentes, y por consiguiente $G$ también lo es, obteniéndose una contradicción.

Presentamos a continuación un bonito y reciente resultado debido a I. M. Isaacs ([40]) sobre los tamaños de clases de los elementos de un grupo $G$ que posee un 
subgrupo normal abeliano. Este resultado sirve además para buscar elementos del grupo con clases de menor tamaño a partir de elementos de los subgrupos normales abelianos. Lo utilizaremos para obtener la conclusion final en el último teorema del capítulo.

Lema 5.3 Sea $G$ un grupo finito y $K$ un subgrupo normal abeliano de $G$. Sea $x$ un elemento no central de $G$ y $t$ un elemento de $K$. Si $y=[t, x]$, entonces $\left|\mathbf{C}_{G}(y)\right|>$ $\left|\mathbf{C}_{G}(x)\right|$, es decir, la $G$-clase de y es más pequeña que la G-clase de $x$.

Demostración. Como $x$ es no central en $G$, el resultado es trivial para $y=1$, por lo que asumiremos que $y \neq 1$.

$\mathrm{Al}$ ser $K \unlhd G$, podemos considerar el subgrupo $H=K \mathbf{C}_{G}(x)$ de $G$. Obsérvese que $y \in K \subseteq H$. Además, $\mathbf{C}_{G}(x) \leq H$, por lo que $\mathbf{C}_{G}(x)=\mathbf{C}_{H}(x)$. Por lo tanto, es suficiente mostrar que $\left|\mathbf{C}_{H}(y)\right|>\left|\mathbf{C}_{H}(x)\right|$ para obtener el resultado.

Aplicando las propiedades de los conmutadores se obtiene que si $u, v \in K$, entonces $[u v, x]=[u, x]^{v}[v, x]=[u, x][v, x]$, donde la última igualdad se verifica porque $[u, x] \in K$, que es abeliano.

Por consiguiente, la aplicación $\theta: K \rightarrow K$ tal que $\theta(k)=[k, x]$, para todo $k \in K$, es homomorfismo cuya imagen, $\theta(K)$, es subgrupo de $K$ de orden

$$
|\theta(K)|=|K: \operatorname{ker} \theta|=\frac{|K|}{\left|\mathbf{C}_{K}(x)\right|}=\frac{|K|}{\left|K \cap \mathbf{C}_{H}(x)\right|}=\frac{\left|K \mathbf{C}_{H}(x)\right|}{\left|\mathbf{C}_{H}(x)\right|}=\left|H: \mathbf{C}_{H}(x)\right| .
$$

Obsérvese que $\theta(K) \unlhd K$. Asimismo, $\mathbf{C}_{G}(x)$ normaliza a $\theta(K)$, ya que si $t_{1} \in K$ y $g \in \mathbf{C}_{G}(x)$ se tiene que $\theta\left(t_{1}\right)^{g}=\left[t_{1}, x\right]^{g}=\left[t_{1}{ }^{g}, x^{g}\right] \in \theta(K)$. Por tanto, $\theta(K) \unlhd$ $K \mathbf{C}_{G}(x)=H$. Como $1 \neq y=\theta(t)$, se tiene que $y^{H}$ es $H$-clase no trivial contenida en $\theta(K)$. Por tanto, $\left|y^{H}\right|<|\theta(K)|=\left|H: \mathbf{C}_{H}(x)\right|$.

Como hemos comentado en la introducción, la estructura de los CP-grupos resolubles fue investigada por Higman en el año 1957 ([36]). Estos grupos poseen un $p$-subgrupo normal $P \neq 1$ para un único primo $p$, tal que el grupo cociente $G / P$ es $p$-grupo ó $\{p, q\}$-grupo, siendo $q$ un primo distinto de $p$. Además, si $G \neq P$ entonces $P=\mathbf{O}_{p}(G)$ y tiene un automorfismo sin puntos fijos de orden primo $q$. En consecuencia, los CP-grupos resolubles no nilpotentes son $\{p, q\}$-grupos con longitud derivada acotada por una constante que depende sólo de $p$ y $q$.

Nosotros utilizaremos concretamente los resultados de Heineken sobre la estructura de los CP-grupos no resolubles y CP-grupos simples que enunciamos a continuación. 
Teorema 5.4 Si $G$ es un CP-grupo finito no resoluble, entonces existen subgrupos normales $B$ y $C$ de $G$ tales que $1 \subseteq B \subseteq C \subseteq G$ y $B$ es 2-grupo, $C / B$ es grupo simple no abeliano y $G / C$ es p-grupo, para algún primo $p$, o cíclico o cuaternio generalizado.

Demostración. Véase la Proposición 2 de ([35]).

A continuación, vamos a introducir el multiplicador de Schur y sus propiedades básicas. Un grupo $G$ se dice que es cuasisimple si es perfecto, es decir $G=G^{\prime}$, y además, $G / \mathbf{Z}(G)$ es simple. Para cada grupo cuasisimple $G$, existe un único grupo simple no abeliano, $S \cong G / \mathbf{Z}(G)$, llamado grupo simple asociado al cuasisimple. Es más, el mayor grupo cuasisimple $\Gamma$ asociado a $S$ es único y se conoce como grupo representación de Schur de $S$. Cualquier otro grupo cuasisimple asociado a $S$ es de la forma $\Gamma / Y$, donde $Y \subseteq \mathbf{Z}(\Gamma)$. Por tanto, el grupo abeliano $M(S)=\mathbf{Z}(\Gamma)$, que se conoce como multiplicador de Schur de $S$, contiene (salvo isomorfía) al centro de cada grupo cuasisimple asociado a $S$. Como ejemplos de grupos cuasisimples podemos citar todos los grupos simples no abelianos, ó también los grupos $\operatorname{SL}(n, q)$, con $n \geq 3$ ó $n=2$ y $q>3$ que, en su mayoría, son grupos no simples.

El segundo resultado de Heineken que vamos a utilizar es una caracterización por isomorfía de los CP-grupos simples no abelianos. El autor obtiene este resultado como conclusión de un análisis exhaustivo de todos los posibles grupos utilizando el Atlas de Grupos Finitos ([25]) y el libro de Gorestein ([32]).

Teorema 5.5 Si $G$ es un CP-grupo finito simple no abeliano, entonces $G$ es isomorfo a uno de los siguientes grupos: $L_{2}(q)$, para $q=5,7,8,9,17, L_{3}(4), S z(8)$ ó $S z(32)$.

Demostración. Véase la Proposición 3 de ([35]).

\subsection{Resultados Principales}

Presentamos ahora los resultados principales del capítulo, en los que se determina la estructura de los subgrupos normales de un grupo $G$ que contienen exactamente dos tamaños de $G$-clases. El primero de ellos muestra que, bajo las hipotesis dadas, el subgrupo normal $N$ de $G$ cuya estructura queremos determinar, es abeliano ó bien todos sus elementos módulo $N \cap \mathbf{Z}(G)$ tienen orden potencia de primo. 
Teorema 5.6 Sea $N$ un subgrupo normal de un grupo finito $G$ y sean 1 y $m$ los únicos tamaños de $G$-clases en $N$, para algún entero $m$. Entonces $N$ es abeliano ó $N /(N \cap \mathbf{Z}(G))$ es un CP-grupo.

Demostración. Denotaremos por $\bar{G}=G /(N \cap \mathbf{Z}(G))$ y utilizaremos barras para trabajar en el grupo cociente. Supongamos que existe $g \in N$ tal que el orden de $\bar{g}$ no es potencia de primo. Entonces existen al menos dos primos distintos $p$ y $q$ tales que la $p$-parte $g_{p}$ de $g$ y la $q$-parte $g_{q}$ de $g$, son elementos de $N$ no centrales en $G$. Por las hipótesis del teorema $\mathbf{C}_{G}(g)=\mathbf{C}_{G}\left(g_{p}\right)=\mathbf{C}_{G}\left(g_{q}\right)$. Dividiremos la demostración en cuatro pasos para llegar a una contradicción.

Paso 1. Se verifica que $\mathbf{C}_{N}(g) \leq \mathbf{Z}\left(\mathbf{C}_{G}(g)\right)$.

Sea $w$ un $q^{\prime}$-elemento de $\mathbf{C}_{N}(g)=\mathbf{C}_{N}\left(g_{q}\right)$ y supongamos que $w$ es no central en $G$. Como $w$ y $g_{q}$ conmutan y son de orden coprimo, se tiene que

$$
\mathbf{C}_{G}\left(w g_{q}\right)=\mathbf{C}_{G}(w) \cap \mathbf{C}_{G}\left(g_{q}\right)=\mathbf{C}_{G}\left(g_{q}\right)=\mathbf{C}_{G}(w)
$$

ya que $w, g_{q}$, y $w g_{q} \in N$, y son no centrales en $G$. Por tanto, $w \in \mathbf{Z}\left(\mathbf{C}_{G}\left(g_{q}\right)\right)=$ $\mathbf{Z}\left(\mathbf{C}_{G}(g)\right)$. De manera análoga se obtiene que si $t$ es un $p^{\prime}$-elemento de $\mathbf{C}_{N}(g)=$ $\mathbf{C}_{N}\left(g_{p}\right)$, entonces $t \in \mathbf{Z}\left(\mathbf{C}_{G}(g)\right)$. Como consecuencia, se tiene que cualquier elemento $z$ del $\mathbf{C}_{N}(g)$ pertenece a $\mathbf{Z}\left(\mathbf{C}_{G}(g)\right)$.

Paso 2. Si $z$ es un elemento de $N \backslash \mathbf{Z}(G)$ tal que $\mathbf{C}_{G}(g) \neq \mathbf{C}_{G}(z)$, entonces $\mathbf{C}_{N}(g) \cap \mathbf{C}_{N}(z)=\mathbf{Z}(G) \cap N$. Es más, $N$ es abeliano ó $\mathbf{Z}(N)=\mathbf{Z}(G) \cap N$.

Sea $z \in N \backslash \mathbf{Z}(G)$ un elemento tal que $\mathbf{C}_{G}(g) \neq \mathbf{C}_{G}(z)$. Supongamos que existe $a \in \mathbf{C}_{N}(g) \cap \mathbf{C}_{N}(z)$, no central en $G$. Entonces, por el Paso $1, a \in \mathbf{Z}\left(\mathbf{C}_{G}(g)\right)$ y $\mathbf{C}_{G}(g) \leq \mathbf{C}_{G}(a)$. Como $a, g \in N$, por hipótesis se tiene que $\mathbf{C}_{G}(a)=\mathbf{C}_{G}(g)$. Aplicando de nuevo el Paso 1 se tiene que $z \in \mathbf{C}_{N}(a)=\mathbf{C}_{N}(g) \leq \mathbf{Z}\left(\mathbf{C}_{G}(g)\right)$. Es decir, $\mathbf{C}_{G}(g) \leq \mathbf{C}_{G}(z)$, y por hipótesis, se da la igualdad, obteniéndose una contradicción. Por tanto $\mathbf{C}_{N}(g) \cap \mathbf{C}_{N}(z)=\mathbf{Z}(G) \cap N$.

Si para todo $z \in N \backslash \mathbf{Z}(G)$ se verifica que $\mathbf{C}_{G}(g)=\mathbf{C}_{G}(z)$, entonces para todo $z \in N \backslash \mathbf{Z}(G)$ se tiene que $\mathbf{C}_{N}(z)=\mathbf{C}_{N}(g)$, y por tanto, $N=\mathbf{C}_{N}(z)$ para todo $z \in N$, es decir, $N$ es abeliano.

Podemos suponer que existe $z \in N \backslash \mathbf{Z}(G)$ tal que $\mathbf{C}_{G}(g) \neq \mathbf{C}_{G}(z)$. Es claro que $\mathbf{Z}(G) \cap N \subseteq \mathbf{Z}(N)$. Por otro lado, de lo demostrado anteriormente se deduce que

$$
\mathbf{Z}(N) \leq \mathbf{C}_{N}(g) \cap \mathbf{C}_{N}(z)=\mathbf{Z}(G) \cap N,
$$


por lo que $\mathbf{Z}(N)=\mathbf{Z}(G) \cap N$.

Para el resto de la demostración supondremos que $N$ es no abeliano, ya que en este caso la prueba termina. Por tanto, asumiremos que $\mathbf{Z}(N)=\mathbf{Z}(G) \cap N$.

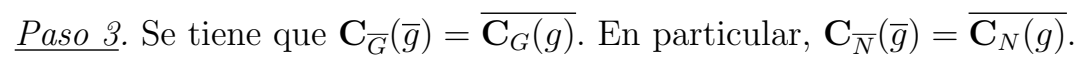

Sabemos que $\overline{\mathbf{C}_{G}(g)} \leq \mathbf{C}_{\bar{G}}(\bar{g})$, para todo $\bar{g} \in \bar{G}$, y que si $\bar{g}=\overline{g_{q}} \overline{g_{q^{\prime}}}$, entonces $\mathbf{C}_{\bar{G}}(\bar{g}) \leq \mathbf{C}_{\bar{G}}\left(\overline{g_{q}}\right)$. Por lo tanto,

$$
\overline{\mathbf{C}_{G}(g)} \leq \mathbf{C}_{\bar{G}}(\bar{g}) \leq \mathbf{C}_{\bar{G}}\left(\overline{g_{q}}\right) .
$$

Como $\mathbf{C}_{\bar{G}}(\bar{g}) \leq \mathbf{C}_{\bar{G}}\left(\overline{g_{q}}\right)$, entonces $\left|\mathbf{C}_{\bar{G}}(\bar{g})\right|$ divide a $\left|\mathbf{C}_{\bar{G}}\left(\overline{g_{q}}\right)\right|$.

Sea $r$ un primo distinto de $q$ y sea $\bar{y} \in \mathbf{C}_{\bar{G}}\left(\overline{g_{q}}\right)$ un elemento de orden potencia de $r$. Entonces $\left[\bar{y}, \overline{g_{q}}\right]=1$, por lo que $\left[y, g_{q}\right] \in \mathbf{Z}(G) \cap N \leq \mathbf{Z}(G)$. Si $o(y)=k$, aplicando el Lema 2.3, se tiene que $1=\left[y^{k}, g_{q}\right]=\left[y, g_{q}\right]^{k}=\left[y, g_{q}{ }^{k}\right]$, es decir $y \in \mathbf{C}_{G}\left(g_{q}{ }^{k}\right)=\mathbf{C}_{G}\left(g_{q}\right)=\mathbf{C}_{G}(g)$, donde la última igualdad se deriva de las hipótesis del teorema. Concluimos que $\bar{y} \in \overline{\mathbf{C}_{G}(g)}$. Por consiguiente, $\left|\mathbf{C}_{\bar{G}}\left(\overline{g_{q}}\right)\right|_{r} \leq\left|\overline{\mathbf{C}_{G}(g)}\right|_{r}$. Como $\left|\overline{\mathbf{C}_{G}(g)}\right|_{r}$ divide a $\left|\mathbf{C}_{\bar{G}}(\bar{g})\right|_{r}$, que a su vez divide a $\left|\mathbf{C}_{\bar{G}}\left(\overline{g_{q}}\right)\right|_{r}$, se tiene que $\left|\overline{\mathbf{C}}_{G}(g)\right|_{r}=\left|\mathbf{C}_{\bar{G}}(\bar{g})\right|_{r}$, para cada primo $r \neq q$. Procediendo de manera análoga con la $p$-parte $g_{p}$ de $g$, se obtiene que $\left|\mathbf{C}_{\bar{G}}(\bar{g})\right|_{r}=\left|\overline{\mathbf{C}_{G}(g)}\right|_{r}$ para cada primo $r \neq p$, y por tanto, $\left|\mathbf{C}_{\bar{G}}(\bar{g})\right|=\left|\overline{\mathbf{C}_{G}(g)}\right|$, de donde $\overline{\mathbf{C}_{G}(g)}=\mathbf{C}_{\bar{G}}(\bar{g})$. G.

Obsérvese que los tres pasos anteriores se verifican para cada conjugado de $g$ en

\section{Paso 4. Conclusión.}

Veamos primero que existe algún $x \in N$, tal que $\bar{g}^{\bar{N}} \cap \overline{\mathbf{C}_{N}(x)}=\emptyset$. Supongamos que para cada $x \in N \backslash \mathbf{Z}(G)$ existe algún $n \in N$ tal que $g^{n} \in \mathbf{C}_{N}(x)$. Entonces $x \in \mathbf{C}_{N}\left(g^{n}\right)=\mathbf{C}_{N}(g)^{n}$ y se tiene que $N=\mathbf{C}_{N}(g)$, por lo que $g \in \mathbf{Z}(N)=\mathbf{Z}(G) \cap N$, y por tanto $g$ es central en $G$, obteniéndose una contradicción.

Consideremos la acción por conjugación de $\overline{\mathbf{C}_{N}(x)}$ sobre el conjunto $\bar{g}^{\bar{N}}$. Veamos que ningún elemento no trivial de $\overline{\mathbf{C}_{N}(x)}$ centraliza a ningún elemento de $\bar{g}^{\bar{N}}$. Si existe un elemento $1 \neq \bar{h} \in \overline{\mathbf{C}_{N}(x)}$, que centraliza a algún cojugado $\overline{g^{t}}$ de $\bar{g}$, para algún $t \in N$, entonces $\bar{h} \in \overline{\mathbf{C}_{N}\left(g^{t}\right)} \cap \overline{\mathbf{C}_{N}(x)}$. Es decir, $\mathbf{C}_{N}\left(g^{t}\right) \cap \mathbf{C}_{N}(x)$ contiene un elemento $h \in N$, no central en $G$. Aplicando el Paso 2 al elemento $g^{t}$ se obtiene que $\mathbf{C}_{G}\left(g^{t}\right)=\mathbf{C}_{G}(x)$. Por consiguiente, $g^{t} \in \mathbf{C}_{G}(x) \cap N=\mathbf{C}_{N}(x)$, por lo que $\overline{g^{t}} \in \bar{g}^{\bar{N}} \cap \overline{\mathbf{C}_{N}(x)}=\emptyset$, y obtenemos una contradicción. 
Por tanto, el estabilizador $S t a b \overline{\mathbf{C}_{N}(x)}\left(\overline{g^{t}}\right)=1$, por lo que la acción es semirregular y $\left|\overline{\mathbf{C}_{N}(x)}\right|$ divide $\left|\bar{g}^{\bar{N}}\right|$, y podemos escribir $\left|\bar{g}^{\bar{N}}\right|=k\left|\overline{\mathbf{C}_{N}(x)}\right|$. Por el Paso 3, se verifica que $\left|\bar{g}^{\bar{N}}\right|=\left|\bar{N}: \overline{\mathbf{C}_{N}(g)}\right|$, por lo que

$$
k\left|\overline{\mathbf{C}_{N}(g)}\right|=|\bar{N}| /\left|\overline{\mathbf{C}_{N}(x)}\right|=|N| /\left|\mathbf{C}_{N}(x)\right|=\left|x^{N}\right| .
$$

Es decir, $\left|\overline{\mathbf{C}_{N}(g)}\right|$ divide a $\left|x^{N}\right|$, que a su vez divide $\left|x^{G}\right|=\left|g^{G}\right|$, y aplicando de nuevo el Paso 3 se obtiene que $\left|\overline{\mathbf{C}_{N}(g)}\right|$ divide $\left|\bar{g}^{\bar{G}}\right|$.

Veamos ahora que $\overline{\mathbf{C}_{N}(g)}$ opera sin puntos fijos, por conjugación, sobre $X=\bar{g}^{\bar{G}} \backslash$ $\bar{g}^{\bar{G}} \cap \overline{\mathbf{C}_{N}(g)}$. Supongamos que existe un elemento $\bar{w} \in \overline{\mathbf{C}_{N}(g)}, \bar{w} \neq \overline{1}$, que centraliza algún elemento $\overline{g^{t}} \in \bar{g}^{\bar{G}}$, para algún $t \in G$. Entonces $\bar{w} \in \mathbf{C}_{\bar{G}}\left(\bar{g}^{\bar{t}}\right)$ y aplicando el Paso 3 se obtiene que $\bar{w} \in \overline{\mathbf{C}_{G}\left(g^{t}\right)}$. Por tanto $\bar{w} \in \overline{\mathbf{C}_{N}(g)} \cap \overline{\mathbf{C}_{N}\left(g^{t}\right)}$. Aplicando ahora el Paso 2 se tiene que $\mathbf{C}_{G}(g)=\mathbf{C}_{G}\left(g^{t}\right)$. Es decir, $\bar{g}^{t} \in \overline{\mathbf{C}_{N}(g)} \cap \bar{g}^{\bar{G}}$ y el estabilizador $S t a b_{\overline{\mathbf{C}_{N}(g)}}\left(\overline{g^{t}}\right)=1$. Por consiguiente, $\left|\overline{\mathbf{C}_{N}(g)}\right|$ divide a $|X|=\left|\bar{g}^{\bar{G}}\right| \backslash\left|\bar{g}^{\bar{G}} \cap \overline{\mathbf{C}_{N}(g)}\right|$. Pero como se ha visto, $\left|\overline{\mathbf{C}_{N}(g)}\right|$ divide $\left|\bar{g}^{\bar{G}}\right|$. Por tanto, concluimos que $\left|\overline{\mathbf{C}_{N}(g)}\right|$ también divide a $\left|\bar{g}^{\bar{G}} \cap \overline{\mathbf{C}_{N}(g)}\right|$, lo que no es posible, ya que

$$
0<\left|\bar{g}^{\bar{G}} \cap \overline{\mathbf{C}_{N}(g)}\right|<\left|\overline{\mathbf{C}_{N}(g)}\right| .
$$

Esta contradicción muestra que todo elemento de $\bar{N}$ es de orden potencia de primo.

El siguiente corolario es una consecuencia inmediata del teorema que acabamos de demostrar.

Corolario 5.7 Sea $N$ un subgrupo normal de un grupo $G$ tal que los tamaños de las $G$-clases de conjugación contenidas en $N$ son 1 y $m$, para algún entero $m$. Entonces $N / \boldsymbol{Z}(N)$ es un $C P$-grupo.

A continuación demostramos el teorema principal del capítulo, en el que probaremos que los subgrupos que estamos estudiando son nilpotentes.

Teorema 5.8 Sea $N$ un subgrupo normal de un grupo finito $G$ y sean 1 y $m$ los únicos tamaños de $G$-clases en $N$, para algún entero $m$. Entonces $N$ es nilpotente.

Demostración. Demostraremos en primer lugar que si $\mathbf{F}(N) \neq \mathbf{Z}(N)$ entonces $N$ es nilpotente. Supongamos que existe un primo $q$ divisor de $|N|$ tal que 
$\mathbf{O}_{q}(N)>\mathbf{Z}(N)_{q}$. Sea $w \in \mathbf{O}_{q}(N) \backslash \mathbf{Z}(N)$ y veamos que $\left|N: \mathbf{C}_{N}(w)\right|$ es potencia de $q$.

Sea $x$ un $r$-elemento de $N$ no central en $G$, para un primo $r \neq q$ y sea $Q$ un $q$-subgrupo de Sylow de $\mathbf{C}_{G}(x)$. Denotaremos por $Q_{0}=\mathbf{O}_{q}(N)=N \cap \mathbf{O}_{q}(G)$. Como $Q_{0} \unlhd G$, podemos considerar la acción del grupo $Q \times\langle x\rangle$ sobre $Q_{0}$. Demostraremos que $\mathbf{C}_{Q_{0}}(Q) \subseteq \mathbf{C}_{Q_{0}}(x)$. Sea $z \in \mathbf{C}_{Q_{0}}(Q)$ no central en $G$, entonces $\langle Q, z\rangle \leq \mathbf{C}_{G}(z)<G$, por lo que $\langle Q, z\rangle=Q$ y $z \in Q \cap Q_{0} \subseteq \mathbf{C}_{G}(x) \cap Q_{0}=\mathbf{C}_{Q_{0}}(x)$.

Aplicando ahora el Teorema 2.10 se obtiene que $\left[Q_{0},\langle x\rangle\right]=1$. Por consiguiente, $x \in \mathbf{C}_{N}\left(Q_{0}\right)$, para todo $r$-elemento $x \in N$, no central en $G$. Se concluye que para cada primo $r \neq q$ el $r$-subgrupo de Sylow de $N$ está en $\mathbf{C}_{N}(w)$, y por tanto, el índice $\left|N: \mathbf{C}_{N}(w)\right|$ es potencia de $q$, como se quería demostrar.

Como $\left(\left|N: \mathbf{C}_{N}(w)\right|,\left|N: Q_{w}\right|\right)=1$, para algún $q$-subgrupo de Sylow $Q_{w}$ de $N$, se tiene que $N=\mathbf{C}_{N}(w) Q_{w}$. Entonces, para cualquier $q^{\prime}$-elemento $y \in \mathbf{C}_{N}(w)$, se verifica que

$$
\mathbf{C}_{G}(y w)=\mathbf{C}_{G}(y) \cap \mathbf{C}_{G}(w)=\mathbf{C}_{G}(w) \subseteq \mathbf{C}_{G}(y)
$$

y por tanto $y \in \mathbf{Z}\left(\mathbf{C}_{G}(w)\right)$. Es decir, $\mathbf{C}_{N}(w)$ factoriza como producto directo de un $q$-subgrupo de Sylow por un subgrupo abeliano, por lo que es nilpotente. En consecuencia, $N$ es resoluble por ser producto de dos subgrupos nilpotentes. Aplicando ahora el Lema 5.2, se concluye que $N$ es nilpotente y el teorema está demostrado.

Podemos suponer, por tanto, que $\mathbf{Z}(N)=\mathbf{F}(N)$, luego $N$ es no resoluble. Veremos que este caso no puede darse. Por el Corolario 5.7, se tiene que $\bar{N}=N / \mathbf{Z}(N)$ es un CP-grupo. Aplicando el Teorema 5.4 a $\bar{N}$, existen subgrupos normales $\bar{B}$ y $\bar{C}$ de $\bar{N}$ tales que $1 \subseteq \bar{B} \subseteq \bar{C} \subseteq \bar{N}$, donde $\bar{B}$ es un 2-grupo, $\bar{C} / \bar{B}$ es simple no abeliano y $\bar{N} / \bar{C}$ es $p$-grupo, para un cierto primo $p$. Como $\mathbf{F}(N / \mathbf{Z}(N))=1$, se tiene que $\bar{B}=1$. Es decir, $\bar{C}$ es un subgrupo normal simple no abeliano y $\bar{N} / \bar{C}$ es $p$-grupo. Por tanto, $\bar{C}=\mathbf{O}^{p}(\bar{N})$ es característico en $\bar{N}$. Como además $\mathbf{Z}(N)$ es característico en $N$, se sigue que $C$ es característico en $N$. Luego $C$ es normal en $G$.

Si $|C|<|N|$, por la hipótesis de inducción se obtiene que $C$ y $\bar{C}$ son subgrupos nilpotentes, y en particular, resolubles. Como $\bar{N} / \bar{C}$ es p-grupo, y por tanto resoluble, entonces $\bar{N}$ es resoluble, obteniéndose una contradicción. Por consiguiente, $C=N$. Luego $\bar{N}$ es simple no abeliano. Por otro lado, si $N^{\prime}<N$ entonces, al ser $N^{\prime}$ característico en $N \unlhd G$, se tiene que $N^{\prime} \unlhd G$ y podemos aplicar inducción para concluir que $N^{\prime}$ es nilpotente. Además $N / N^{\prime}$ es abeliano, y por tanto $N$ es resoluble, obteniéndose de nuevo una contradicción. Por tanto, $N^{\prime}=N$, es decir, $N$ es perfecto. Como además $N / \mathbf{Z}(N)$ es simple no abeliano, se tiene que $N$ es cuasisimple. 
Aplicando el Teorema 5.5 se obtiene que $N / \mathbf{Z}(N)$ es isomorfo a uno de los siguientes grupos: $L_{2}(q)$, con $q=5,7,8,9,17, L_{3}(4), S z(8)$ ó $S z(32)$. Es fácil comprobar ([25]) que los Multiplicadores de Schur de estos grupos tienen los siguientes órdenes: para los grupos $L_{2}(8)$ y $S z(32)$ el orden es $|M(S)|=1$, para los grupos $L_{2}(5), L_{2}(7)$ y $L_{2}(17)$ el orden es $|M(S)|=2$, para el grupo $S z(8)$ el orden es $|M(S)|=4$, para el grupo $L_{2}(9)$ el orden es $|M(S)|=6$ y para el grupo $L_{3}(4)$ el orden es $|M(S)|=48$. Por tanto, en todos los casos, el Multiplicador de Schur del grupo simple es 2-grupo ó $\{2,3\}$-grupo. Como $\mathbf{Z}(N) \subseteq M(S)$, se tiene que $\mathbf{Z}(N)$ es 2-grupo ó $\{2,3\}$-grupo también. Veamos que esto implica que $\bar{N}$ es resoluble.

Demostraremos que $|\bar{N}|$ divide $|\mathbf{Z}(N)|$. Comprobaremos primero que $|\bar{N}|$ divide a $m$. Por el Teorema 5.6, se tiene que $N /(N \cap \mathbf{Z}(G))$ es CP-grupo, y en particular $\bar{N}$ es CP-grupo también. Entonces, para todo primo $p$ divisor de $|\bar{N}|$ existe un $p$-elemento $x$ no central de $N$. Si $y$ es $p^{\prime}$-elemento de $\mathbf{C}_{N}(x) \backslash \mathbf{Z}(N)$, entonces $\overline{x y}$ es elemento compuesto de $\bar{N}$, lo que no puede darse. Por lo tanto, $\mathbf{C}_{N}(x)=\mathbf{C}_{N}(x)_{p} \times \mathbf{Z}(N)_{p^{\prime}}$, siendo $\mathbf{C}_{N}(x)_{p}$ el $p$-subgrupo de Sylow de $\mathbf{C}_{N}(x)$, y se tiene que

$$
|\bar{N}|_{p^{\prime}}=|N: \mathbf{Z}(N)|_{p^{\prime}}=\frac{|N|_{p^{\prime}}}{|\mathbf{Z}(N)|_{p^{\prime}}}=\frac{|N|_{p^{\prime}}}{\left|\mathbf{C}_{N}(x)\right|_{p^{\prime}}}=\left|N: \mathbf{C}_{N}(x)\right|_{p^{\prime}}
$$

Es decir, $\left|x^{N}\right|_{p^{\prime}}=\left|N: \mathbf{C}_{N}(x)\right|_{p^{\prime}}=|\bar{N}|_{p^{\prime}}$, y como $\left|x^{N}\right|_{p^{\prime}}$ divide $m_{p^{\prime}}$, se sigue que $|\bar{N}|_{p^{\prime}}$ también divide $m_{p^{\prime}}$. Considerando todos los primos divisores de $|\bar{N}|$, se concluye que $|\bar{N}|$ divide a $m$. Dado que todo elemento de $N$ es central ó pertenece a una $G$-clase de tamaño $m$, utilizando la ecuación de clases se obtiene que

$$
|N|=|\mathbf{Z}(G) \cap N|+m k \text {, para un entero } k \text {. }
$$

Como $|\bar{N}|$ divide $m$, se sigue que también divide $|\mathbf{Z}(G) \cap N|$. En particular, $|\bar{N}|$ divide $|\mathbf{Z}(N)|$ que, como hemos visto, es $\{2\}$-grupo ó $\{2,3\}$-grupo. Es decir, $\bar{N}$ es también $\{2\}$-grupo ó $\{2,3\}$-grupo, y por tanto resoluble. Por consiguiente, este caso no puede darse y así $N$ es nilpotente.

Como consecuencia del Teorema 5.8 obtenemos el siguiente corolario:

Corolario 5.9 Supongamos que $N$ es un subgrupo normal de un grupo $G$ tal que los tamaños de $G$-clases de $N$ son 1 y $m$, para algún entero $m$. Entonces $N$ es abeliano ó $N=P \times A$, con $P$ un p-subgrupo de $N$ y A central en $G$.

Demostración. Por el Teorema 5.8 sabemos que $N$ es nilpotente. Si $N$ no es abeliano, aplicando el Teorema 5.6, se tiene que $N /(\mathbf{Z}(G) \cap N)$ es $p$-grupo, para algún primo $p$, y el resultado se sigue. 
Es fácil construir ejemplos de los dos casos que aparecen en el Corolario 5.9. Sea $N$ un grupo abeliano de orden impar y sea $\alpha$ el automorfismo involutivo de $N$. Entonces $N$ es un subgrupo normal abeliano de $G=N\langle\alpha\rangle$ que contiene $G$-clases de tamaños 1 ó 2. Por otro lado, si $Q$ es el grupo cuaternio de orden 8 y $\beta \in \operatorname{Aut}(Q)$ es de orden 3, entonces $Q$ es subgrupo normal de $G=Q\langle\beta\rangle$ y los tamaños de las $G$-clases contenidas en $Q$ son 1 ó 6 . Este es un ejemplo de un $p$-subgrupo normal no abeliano de $G$ que contiene exactamente dos tamaños de $G$-clases.

Varios autores (I.M. Isaacs [41], A. Mann [51], L. Verardi [65]) han demostrado independientemente que si $G$ es un $p$-grupo con dos tamaños de clases de conjugación, entonces $G / \mathbf{Z}(G)$ tiene exponente $p$. En el siguiente teorema extendemos este resultado para un $p$-subgrupo normal $P$ con dos tamaños de $G$-clases, y en particular, desarrollamos una demostración alternativa para el caso $P=G$. La demostración se basa en la construcción de un subgrupo normal abeliano de $P$ con determinadas propiedades y se inspira en la demostración de la Proposición 2.2 de [43].

Teorema 5.10 Sea $G$ un grupo finito y sea $P$ un p-subgrupo normal no abeliano de $G$, con dos tamaños de $G$-clases de conjugación. Entonces $P /(\mathbf{Z}(G) \cap P)$, y en particular $P / \mathbf{Z}(P)$, tienen exponente $p$.

Demostración. Como $\mathbf{Z}(G) \cap P \subseteq \mathbf{Z}(P)$, si $P /(\mathbf{Z}(G) \cap P)$ tiene exponente $p$, entonces $P / \mathbf{Z}(P)$ tiene trivialmente exponente $p$. Por tanto, sólo necesitamos demostrar que para todo $x \in P$ se verifica que $x^{p} \in \mathbf{Z}(G)$. Supongamos que el Teorema no es cierto. Sea $x$ un elemento de $P$ tal que $x^{p} \notin \mathbf{Z}(G)$. Denotaremos por $Z_{1}=\mathbf{Z}(P)$. Como $P$ es $p$-grupo no abeliano, podemos considerar el subgrupo $Z_{2}$ tal que $Z_{2} / Z_{1}=\mathbf{Z}\left(P / Z_{1}\right)$. Obsérvese que $Z_{2}$ char $P \unlhd G$, y por tanto, $Z_{2} \unlhd G$. Consideremos el subgrupo $T_{2}=\mathbf{C}_{P}\left(Z_{2}\right)<\mathbf{C}_{P}\left(Z_{1}\right)=P$. Es fácil ver que $T_{2} \unlhd G$. Dividiremos la demostración en varios pasos.

Paso 1. Si $z \in P \backslash T_{2}$, entonces $z^{p} \in \mathbf{Z}(G)$. Por lo tanto, $x \in T_{2}$.

Como $z \notin \mathbf{C}_{P}\left(Z_{2}\right)$, existe algún elemento $y \in Z_{2}$ tal que $1 \neq[y, z] \in\left[Z_{2}, P\right] \leq$ $Z_{1}$. De las hipótesis del teorema se deduce que $z^{p} \in \mathbf{Z}(G)$ ó $\mathbf{C}_{P}\left(z^{p}\right)=\mathbf{C}_{P}(z)$. Mostraremos que el segundo caso no puede darse. Sea $p^{a}$ el orden de $[y, z]$. Aplicando el Lema 2.3 se tiene que

$$
1=[y, z]^{p^{a}}=\left([y, z]^{p}\right)^{p^{a-1}}=\left[y, z^{p}\right]^{p^{a-1}}=\left[y^{p^{a-1}}, z^{p}\right]
$$

y por tanto, $y^{p^{a-1}} \in \mathbf{C}_{G}\left(z^{p}\right)=\mathbf{C}_{G}(z)$. Aplicando de nuevo el Lema $2.3,\left[y^{p^{a-1}}, z\right]=$ $[y, z]^{p^{a-1}}=1$, obteniéndose una contradicción. Concluimos que $z^{p} \in \mathbf{Z}(G) \mathrm{y}$, en 
particular, $x \in T_{2}$.

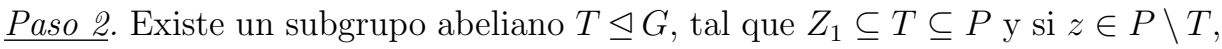
entonces $z^{p} \in \mathbf{Z}(G)$. Como consecuencia se tiene que $x \in T$.

Si $T_{2}$ es abeliano entonces $T=T_{2}$ y el paso está demostrado. Supongamos por tanto que $T_{2}$ es no abeliano. Como $T_{2} / \mathbf{Z}\left(T_{2}\right)$ es $p$-grupo, podemos considerar el subgrupo no trivial $Z_{3}$ tal que $Z_{3} / \mathbf{Z}\left(T_{2}\right)=\mathbf{Z}\left(T_{2} / \mathbf{Z}\left(T_{2}\right)\right)$. Denotaremos por $T_{3}=\mathrm{C}_{T_{2}}\left(Z_{3}\right)<T_{2}$. Obsérvese que $Z_{1} \subseteq T_{3} \subseteq T_{2} \subseteq P$. Es más, se tiene que $Z_{3} / \mathbf{Z}\left(T_{2}\right)$ char $T_{2} / \mathbf{Z}\left(T_{2}\right)$, por lo que $Z_{3}$, y por tanto $T_{3}$, son normales en $\mathrm{G}$.

Si $z \in P \backslash T_{3}$, entonces $z \in P \backslash T_{2}$ ó $z \in T_{2} \backslash T_{3}$. En el primer caso, aplicando el Paso 1, se obtiene que $z^{p} \in \mathbf{Z}(G)$. En el segundo caso se obtiene que $z \notin \mathbf{C}_{P}\left(Z_{3}\right)$, por consiguiente, existe un elemento $y \in Z_{3}$ tal que $1 \neq[y, z] \in\left[Z_{3}, T_{2}\right] \leq \mathbf{Z}\left(T_{2}\right)$. Argumentando como en el Paso 1 , se obtiene que $z^{p} \in \mathbf{Z}(G)$.

Si $T_{3}$ es abeliano, tomamos $T=T_{3}$ y el paso está probado. En caso contrario, repetimos el procedimiento para construir, a partir de $Z_{3}$, los subgrupos $Z_{4}$ y $T_{4}=\mathbf{C}_{P}\left(Z_{4}\right)$ tales que para todo $z \in P \backslash T_{4}$ se verifica que $z^{p} \in \mathbf{Z}(G)$. Este método proporciona una cadena descendente de subgrupos $T_{i} \unlhd G$, tales que $Z_{1} \subseteq T_{i} \subseteq P$, y que verifican que si $z \in P \backslash T_{i}$ entonces $z^{p} \in \mathbf{Z}(G)$. El hecho de que $Z_{1}$ es abeliano nos permite asegurar que existe un $T_{i}$ abeliano, para algún $i$, y podemos hacer $T=T_{i}$, obteniéndose el resultado deseado.

Paso 3. Si $z \in T$ y $z^{p} \notin \mathbf{Z}(G)$, entonces $\mathbf{C}_{P}(z)=T$.

Como $T$ es abeliano, trivialmente se sigue que $T \subseteq \mathbf{C}_{P}(z)$. Supongamos ahora que existe un elemento $y \in \mathbf{C}_{P}(z) \backslash T$. Entonces $y \in P \backslash T$ y, por el Paso 2 se tiene que $y^{p} \in \mathbf{Z}(G)$. Por tanto, $y^{p} z^{p}=(y z)^{p} \notin \mathbf{Z}(G)$, por lo que, aplicando de nuevo el Paso 2, se obtiene que $y z \in T$, y por tanto $y \in T$, y llegamos a una contradicción.

Paso 4. Si $\bar{G}=G /(\mathbf{Z}(G) \cap P)$, entonces $\overline{\mathbf{C}_{P}(x)}=\mathbf{C}_{\bar{P}}(\bar{x})$.

Es trivial ver que $\overline{\mathbf{C}_{P}(x)} \subseteq \mathbf{C}_{\bar{P}}(\bar{x})$. Consideremos por tanto, un elemento $y$ de $P$ tal que $\bar{y} \in \mathbf{C}_{\bar{P}}(\bar{x})$. Si $y^{p} \notin \mathbf{Z}(G)$ entonces, por el Paso 2 , se tiene que $y \in T, \mathrm{y}$ por el Paso 3, el $\mathbf{C}_{P}(y)=T=\mathbf{C}_{P}(x)$, por lo que $y \in \mathbf{C}_{P}(x)$. Si $y^{p} \in \mathbf{Z}(G)$, como $[x, y]=1$, se tiene que $x^{p} y^{p}=(x y)^{p} \in \mathbf{Z}(G)$. Aplicando el Paso 2 se deduce que $x y \in T$, y por tanto, $y \in T=\mathbf{C}_{P}(x)$. Por consiguiente $\bar{y} \in \overline{\mathbf{C}_{P}(x)}$, y se tiene que $\mathbf{C}_{\bar{P}}(\bar{x}) \subseteq \overline{\mathbf{C}_{P}(x)}$, como se quería demostrar. 
Paso 5. Conclusión.

Sea $g \in P \backslash T$. Como $x \in T$ y $g$ es no central en $P$, podemos aplicar el Lema 5.3 al elemento $y=[x, g]$, y concluir que $\left|y^{G}\right|<\left|g^{G}\right|$. Por las hipótesis del teorema se tiene $\left|y^{G}\right|=1$, es decir, $y$ es central en $G$. En consecuencia, $\bar{y}=1=[\bar{x}, \bar{g}] \in$ $\mathbf{C}_{\bar{P}}(\bar{x})=\overline{\mathbf{C}_{P}(x)}$, donde la última igualdad resulta de aplicar el Paso 4. Por tanto $g \in \mathbf{C}_{P}(x)=T$, obteniéndose una contradicción. Esto demuestra que no existe tal elemento $x \in P$, y que $\bar{P}$ tiene exponente $p$, lo que concluye la demostración. 



\section{Bibliografía}

[1] Akhlaghi, Z., Beltrán, A., Felipe, M.J., Khatami, M. Structure of normal subgroups with three $G$-class sizes. Monatshefte fur Mathematik (21 February 2011) 1-12. doi:10.1007/s00605-011-0290-8.

[2] Alemany, E., Beltrán, A., Felipe, M.J. Finite groups with two p-regular conjugacy class lengths II. Bulletin of the Australian Mathematical Society 79 (2009) 419-425.

[3] Alemany, E., Beltrán, A., Felipe, M.J. Nilpotency of normal subgroups having two G-class sizes. Proc. Am. Math. Soc. 139 (2011) 2663-2669.

[4] Alemany, E., Beltrán, A., Felipe, M.J. Itô's theorem on groups with two class sizes revisited. Actualmente sometido.

[5] Arad, Z., Fisman, E. A proof of Szep's conjecture on nonsimplicity of certain finite groups. Journal of Algebra 108 (1987) no 2 240-354.

[6] Baer, R. Group elements of prime power index. Trans. Amer. Math. Soc. 75 (1953), 20-47.

[7] Beltrán, A., Felipe, M.J. Finite groups with two p-regular conjugacy class lengths. Bulletin of the Australian Mathematical Society 67 (2003) 163-169.

[8] Beltrán, A., Felipe, M.J. Certain relations between p-regular class sizes and the p-structure of p-solvable groups. Journal of the Australian Mathematical Society 77 (2004) no 3 387-400.

[9] Beltrán, A., Felipe, M.J. Prime factors of $\pi$-partial character degrees and conjugacy class sizes of $\pi$-elements. Algebra Colloquium. 12 (2005) no 4 699-707.

[10] Beltrán, A., Felipe, M.J. Variations on a theorem by Alan Camina on conjugacy class sizes. Journal of Algebra. 296 (2006) 253-266.

[11] Beltrán, A., Felipe, M.J. Some class size conditions implying solvability of finite groups. Journal of Group Theory. 9 (2006) no 6 787-797. 
[12] Beltrán, A., Felipe, M.J. Nilpotency of p-complements and p-regular conjugacy class sizes. Journal of Algebra. 308 (2007) no 2 641-653.

[13] Beltrán, A., Felipe, M.J. Conjugacy classes of p-regular elements in p-solvable groups. Proceedings Group St. Andrews. Ed. Cambridge. 1 (2007) 224-229.

[14] Beltrán, A., Felipe, M.J. Structure of finite groups under certain arithmetical conditions on class sizes. Journal of Algebra. 319 (2008) no 3 897-910.

[15] Beltrán, A., Felipe, M.J. The structure of finite groups with three class sizes. J. Group Theory. 12 (2009) no 3 539-553.

[16] Beltrán, A., Felipe, M.J. Finite groups with four conjugacy class sizes. Communications in Algebra. 39 (2011) no 4 1260-1272.

[17] Bertram, E.A., Herzog. M, Mann, A. On a graph related to conjugacy classes of groups. Bull. London Math. Soc. 22 (1990) no 6 569-575.

[18] Bianchi, M., Gillio, A., Casolo, C. A note on conjugacy class sizes of finite groups. Rend. Sem. Mat. Padova. 106 (2001) 255-260.

[19] Bianchi, M.G., Chillag, D., Mauri, A., Herzog, M., Scoppola, C. Applications of a graph related to conjugacy classes of finite groups. Arch. Math. 58 (1992) no 2 126-132.

[20] Burnside, W. On groups of order $p^{a} q^{b}$. Proc. Lond. Math. Soc. 2 (1904) 388-392.

[21] Camina, A.R. Arithmetical conditions on the conjugacy class numbers of a finite group. J. London Math. Soc. 2 (1972) no 5 127-132.

[22] Camina, A.R. Finite groups of conjugate rank 2. Nagoya Math. J. 53 (1974) 47-57.

[23] Camina, A.R., Camina, R.D. Implications of conjugacy class size. J. Group Theory 1 (1998), 257-269.

[24] Camina, A.R., Camina, R.D. Coprime conjugacy class sizes. Asian-Eur. J. Math., 2(2)(2009), 183-190.

[25] Conway, J., Curtis, R., Norton, S., Parker, R., Wilson, R. The Atlas of Finite Groups. Oxford University Press, New York (1985).

[26] Chillag, D., Herzog, M. On the lengths of the conjugacy classes of finite groups. J. Algebra 131 (1990), 110-125. 
[27] Chillag, D., Mann, A. Nearly odd-order and nearly real finite groups. Common Algebra. 26 (1998) 2041-2064.

[28] Cossey, J., Wang, Y. Remarks on the length of conjugacy classes of finite groups. Communications in Algebra 27 (9) (1999) 4347-4353.

[29] Dolfi, S., Jabara E. The structure of finite groups of conjugate rank 2. Bull. London Math. Soc., 41(5) (2009), 916-926.

[30] Dolfi, S., Pacifici, E., Sanus, L. Finite groups with real conjugacy classes of prime size. Israel J. Math. 175 (2010) 179-189.

[31] Fein, B., Kantor, W.M., Schacher, M. Relative Brauer groups, II J. Reine Angew. Math., 328 (1981) 39-57.

[32] Gorenstein, D. Finite simple groups. Plenum Press, New York-London (1982).

[33] Gorenstein, D., Walter, J.H. On finite groups with dihedral Sylow 2-subgroups. Illinois J. Math. 6 (1962) 553-593.

[34] Guo, X., Zhao, X. On the normal subgroups with exactly two G-conjugacy class sizes. Chin. Ann. Math. B 30 (2009) 427-432.

[35] Heineken, H. On groups all of whose elements have prime power order. Math. Proc. R. Ir. Acad. 106A (2006) no 2 191-198.

[36] Higman, G. Finite groups in which every element has prime power order. Journal London Math. Soc. 32 (1957) 335-342.

[37] Huppert, B. Endliche Gruppen I. Springer-Verlag. Berlín-Heidelberg-New York (1967).

[38] Huppert, B. Character Theory of Finite Groups. Walter de Gruyter and Co. Berlín, 1998.

[39] Isaacs, I.M. Algebra: A Graduate Course. Brooks/Cole Publishing Company (1994).

[40] Isaacs, I.M. Subgroups generated by small classes in finite groups. Proc. Amer. Math. Soc. 136 (2008) no 7 2299-2301.

[41] Isaacs, I.M. Groups with many equal classes. Duke Math J. 37 (1970) 501-506.

[42] Ishikawa, K. On finite p-groups which have only two conjugacy class lengths. Israel J. Math. 129 (2002) 119-123. 
[43] Ito, N. On finite groups with given conjugate type I. Nagoya Math. J. 6 (1953) 17-28.

[44] Ito, N. On finite groups with given conjugate type II. Osaka J. Math. 7 (1970) 231-251.

[45] Iwasaki, S. On finite groups with exactly two real conjugate classes. Arch. Math. (Basel) 33(6) (1979) 512-517.

[46] Kazarin, L.S. Burnside $p^{\alpha}$-lemma. Mathemat Notes 48 (1990), translated from Matematicheskie Zamatki 48 (1990), 45-48.

[47] Kazarin, L.S. On groups with isolated conjugacy classes. Izv. Vyssh. Uchebn. Zaved. Mat. 7 (1981) 40-45.

[48] Knoche, H.G. Uber den Frobenius'schen Klassengegriff in nilpotent Grouppen. Math. Z. 55 (1951) 71-83.

[49] Kurzweil, H., Stellmacher, B. The Theory of Finite Groups, an introduction. Universitext, Springer (2004).

[50] Liu, X., Wang, Y., Wei, H. Notes on the length of conjugacy classes of finite groups. J. Pure Appl. Algebra 196 (2005) no 1, 111-117.

[51] Mann, A. Conjugacy classes in finite groups. Israel J. Math. 31 (1978) 78-84.

[52] Moreto, A., Navarro, G. Groups with three real valued irreducible characters. Israel J. Math. 163 (2008) no 1 85-92.

[53] Navarro, G., Sanus L. Rationality and normal 2-complements. J. Algebra 320 (2008) no 6 2451-2454.

[54] Ninomiya, Y. Structure of p-solvable groups with three p-regular classes. Canad. J. Math. 43 (1991) no 3 559-579.

[55] Ninomiya, Y. Structure of p-solvable groups with three p-regular classes II. Math. J. Okayama Univ. 35 (1993) 29-34.

[56] Ren, Y.C. On the length of p-regular classes and the p-structure of finite groups. Algebra Colloq. 2 (1995) no 1 3-10.

[57] Rebmann, J. F-Gruppen. Arch. Math. (Basel) 22 (1971) 225-230.

[58] Rose, J.S. A course on Group Theory. Dover First Edition. New York (1994).

[59] Robinson, J.S. A course in the Theory of Groups. Graduate Texts in Mathematics 80. Second Edition. Springer-Verlag. Berlin-Heidelberg-New York (1995). 
[60] Sangroniz, J., Tent, J. 2-groups with few rational conjugacy classes. J. of Algebra (2011). doi:10.1016/j.jalgebra.2011.01.023.

[61] Shirong, L. On the class length of elements of prime power order in finite groups. Guangxi Sci. 6 (1999) no. 1 12-13.

[62] Shirong, L. Finite groups with exactly two class lengths of elements of prime power order. Arch Math. 67 (1996) 100-105.

[63] Sylow, M.L. Théorèmes sur les groupes de substitutions. Math. Ann. 5 (1872) no 4 584-594.

[64] The GAP Group, GAP-groups, Algorithms and Programming, Vers. 4.4.12 (2008), http://www.gap-system.org.

[65] Verardi, L. On groups whose noncentral elements have the same finite number of conjugates. Boll. Un. Mat. Ital. A(7) 2 (1988) no 4 391-400.

[66] Zhao, X., Guo, X. On Conjugacy Class Sizes of the p'-Elements with Prime Power Order. Algebra Colloquium 16:4 (2009) 541-548. 



\section{Notación}

$\begin{array}{ll}A, X, Y & \text { denotan siempre conjuntos finitos } \\ Y \subseteq X & Y \text { es un subconjunto de } X \\ X \times Y & \text { producto cartesiano de los conjuntos } X \text { e } Y \\ G, N, H, L, K & \text { denotan siempre grupos finitos } \\ H \leq G & H \text { es un subgrupo de } G \\ H<G & H \text { es un subgrupo propio de } G \\ K \unlhd G & K \text { es un subgrupo normal de } G \\ K \text { char } G & K \text { es un subgrupo característico de } G \\ G / K & \text { grupo cociente de } G \text { por } K \text { (donde } K \unlhd G \text { ) } \\ H K & \text { subgrupo producto de los subgrupos } H \text { y } K \text { de } G \\ H \times K & \text { producto directo de los subgrupos } H \text { y } K \\ G_{1} \times G_{2} \times \ldots \times G+ & \text { producto directo de los grupos } G_{1}, G, \ldots, G_{n} \\ \mathbf{C}_{G}(x) & \text { centralizador de } x \text { en } G, \text { donde } x \in G \\ \mathbf{C}_{G}(A) & \text { centralizador de } A \text { en } G \text {, donde } A \subseteq G \\ \mathbf{C}_{G}(H) & \text { centralizador de } H \text { en } G \text {, donde } H \leq G \\ \mathbf{C}_{G}(H / K) & \text { centralizador de } H / K \text { en } G \text { (donde } K \unlhd G \text { y } K \leq H \leq G \text { ) } \\ \mathbf{N}_{G}(A) & \text { normalizador de } A \text { en } G \text {, donde } A \subseteq G \\ \mathbf{N}_{G}(H) & \text { normalizador de } H \text { en } G \text {, donde } H \leq G \\ \mathbf{Z}_{(}(G) & \text { centro de } G \\ G^{\prime} & \text { grupo derivado de } G \\ \operatorname{Syl}_{\mathrm{p}}(G) & \text { conjunto de } p \text {-subgrupos de Sylow de } G\end{array}$




\begin{tabular}{|c|c|}
\hline $\mathrm{O}_{r}(G)$ & $r$-radical de $G$, para un cierto primo $r$ \\
\hline $\mathbf{O}^{r}(G)$ & $r$-residual de $G$, para un cierto primo $r$ \\
\hline $\mathbf{O}_{r^{\prime}, r}(G)$ & $\begin{array}{l}\text { subgrupo de } G \text { tal que } \mathbf{O}_{r^{\prime}, r}(G) / \mathbf{O}_{r^{\prime}}(G) \text { es el } r \text {-radical del } \\
\text { grupo cociente } G / \mathbf{O}_{r^{\prime}}(G)\end{array}$ \\
\hline$F(G)$ & subgrupo Fitting de $G$ \\
\hline$[H, K]$ & $\begin{array}{l}\text { subgrupo conmutador de } G \text {, generado por los elementos } \\
\{[h, k]: h \in H, k \in K\}\end{array}$ \\
\hline$\langle H, K\rangle$ & $\begin{array}{l}\text { subgrupo generado por la unión de los subgrupos } H \text { y } K \text { de } \\
G\end{array}$ \\
\hline$\langle X\rangle$ & subgrupo de $G$ generado por $X(\subseteq G)$ \\
\hline$\left\langle x_{1}, \ldots, x_{n}\right\rangle$ & subgrupo generado por los elementos $\left\{x_{1}, \ldots, x_{n}\right\}$ \\
\hline$M(S)$ & Multiplicador de Schur del grupo simple $S$ \\
\hline$G^{\prime}, G^{\prime \prime}, G^{\prime \prime \prime}, \ldots, G^{(n)}$ & términos de la serie derivada de $G$ \\
\hline$Q_{8}$ & grupo quaternio de orden 8 \\
\hline$G \backslash A$ & conjunto de elementos de $G$ que no están en $A$ \\
\hline$g H$ & coclase izquierda de $H$ en $G$ conteniendo $g \in G$ \\
\hline$p$ & un número primo \\
\hline$p^{\prime}$ & conjunto de números primos distintos de $p$ \\
\hline$\pi$ & un conjunto de números primos \\
\hline$\pi^{\prime}$ & el conjunto de números primos que no pertenecen a $\pi$ \\
\hline p-número & entero positivo divisible únicamente por el primo $p$ \\
\hline$p^{\prime}$-número & entero positivo no divisible por el primo $p$ \\
\hline $\mathbb{Z}^{+}$ & el conjunto de los números enteros positivos \\
\hline$(a, b)$ & máximo común divisor de los enteros $a$ y $b$ \\
\hline$o(g)$ & orden de un elemento de un grupo \\
\hline$|X|$ & número de elementos en el conjunto $X$ \\
\hline$|G|$ & orden del grupo $G$ \\
\hline$\pi(G)$ & conjunto de primos que divide a $|G|$ \\
\hline$\left|x^{G}\right|_{p_{i}}$ & $\begin{array}{l}p_{i} \text {-parte de la factorización de }\left|x^{G}\right| \text { como producto de poten- } \\
\text { cias de números primos }\end{array}$ \\
\hline
\end{tabular}




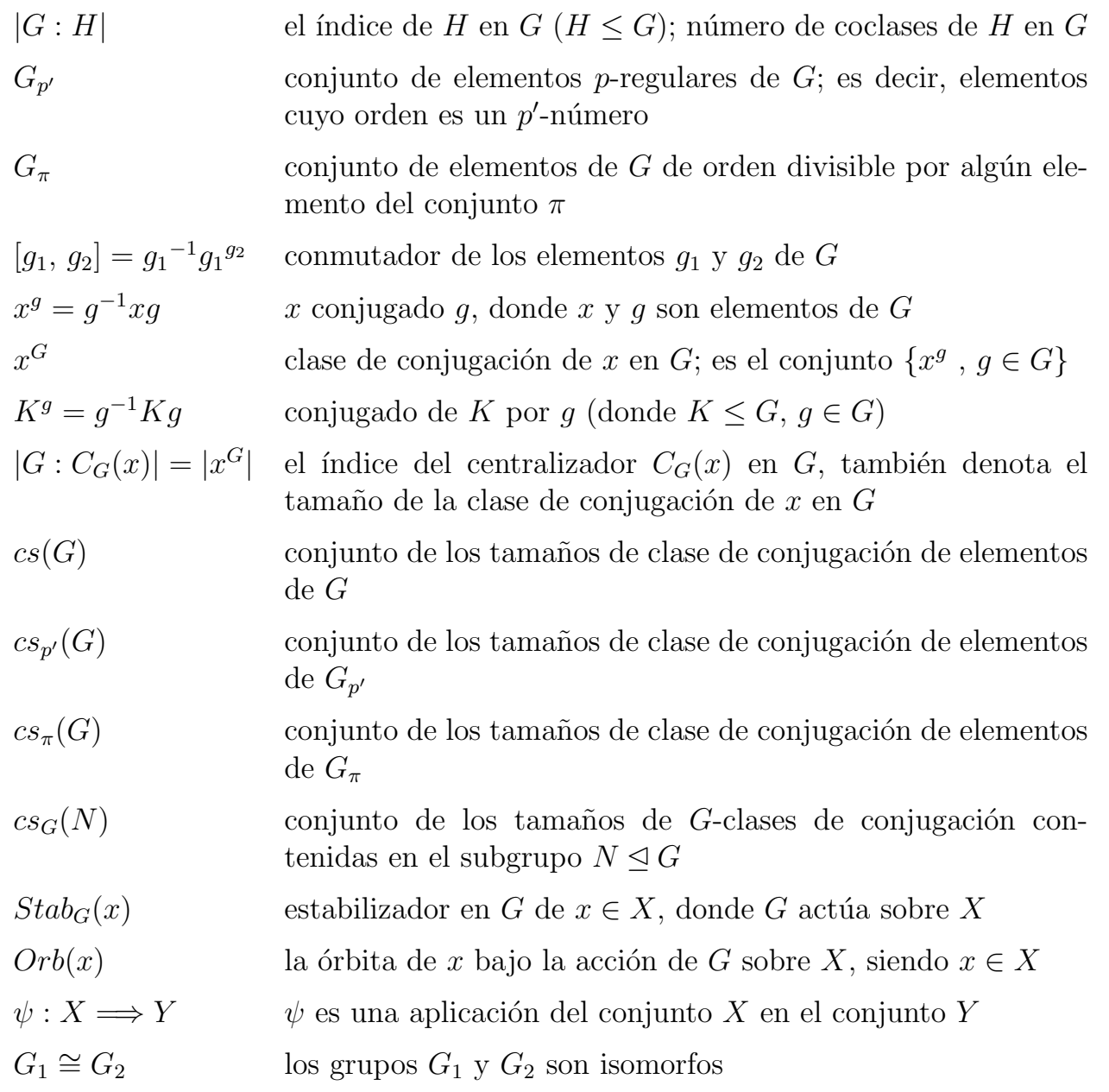

